\title{
Ultracold Dipolar Gases in Optical Lattices
}

\section{Christian Trefzger}

Thesis Advisor:

Prof. Maciej Lewenstein

Thesis Co-advisor:

Dr. Chiara Menotti
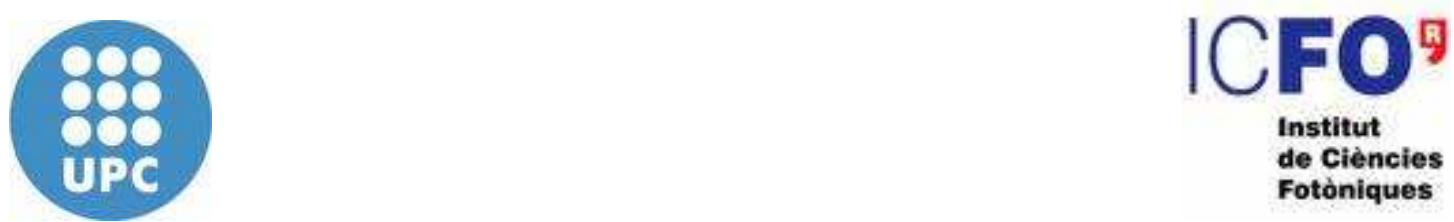


\section{General introduction}

This thesis is a theoretical work, in which we study the physics of ultra-cold dipolar bosonic gases in optical lattices. Such gases consist of bosonic atoms or molecules, cooled below the quantum degeneracy temperature, typically in the $\mathrm{nK}$ range. In such conditions, in a three-dimensional (3D) harmonic trap, weakly interacting Bosons condense and form a Bose-Einstein Condensate (BEC). When a BEC is loaded into an optical lattice produced by standing waves of laser light, new kinds of physical phenomena occur. These systems realize then Hubbard-type models and can be brought to a strongly correlated regime.

In 1989, M. Fisher et. al. predicted that the homogeneous Bose-Hubbard model (BH) exhibits the Superfluid-Mott insulator (SF-MI) quantum phase transition [1]. In 2002 the transition between these two phases were observed experimentally for the first time in the group of I. Bloch, T. Esslinger and T. Hänsch [2]. The experimental realization of a dipolar BEC of Chromium by the group of $\mathrm{T}$. Pfau $[3,4,5]$, and the recent progresses in trapping and cooling of dipolar molecules by the groups of D. Jin and J. Ye $[6,7,8]$, have opened the path towards ultra-cold quantum gases with dominant dipole interactions. A natural evolution, and present challenge, on the experimental side is then to load dipolar BECs into optical lattices and study strongly correlated ultracold dipolar lattice gases.

Before this PhD work, studies of $\mathrm{BH}$ models with interactions extended to nearest neighbors had pointed out that novel quantum phases, like supersolid (SS) and checkerboard phases (CB) are expected $[9,10,11,12]$. Due to the long-range character of the dipole-dipole interaction, which decays as the inverse cubic power of the distance, it is necessary to include more than one nearest neighbor to have a faithful quantitative description of dipolar systems. In fact, longer-range interactions tend to allow for and stabilize more novel phases.

In this thesis we first study $\mathrm{BH}$ models with dipolar interactions, going beyond the ground state search. We consider a two-dimensional (2D) lattice where the dipoles are polarized perpendicularly to the 2D plane, resulting in 
an isotropic repulsive interaction. We use the mean-field approximations and a Gutzwiller Ansatz which are quite accurate and suitable to describe this system. We find that dipolar bosonic gas in 2D lattices exhibits a multitude of insulating metastable states, often competing with the ground state, similarly to a disordered system. We study in detail the fate of these metastable states: how can they be prepared on demand, how they can be detected, what is their lifetime due to tunneling, and what is their role in various cooling schemes. Moreover, we find that the ground state is characterized by insulating checkerboard-like states with fractional filling factors $\nu$ (average number of particles per site) that depend on the cut-off used for the interaction range. We confirm this prediction by studying the same system with Quantum Monte Carlo methods (the worm algorithm). In this case no cut-off for the dipolar interaction is used, and we find evidence for a Devil's staircase in the ground state, i.e. insulating phases which appear at all rational $\nu$ of the underlying lattice. We also find regions of parameters where the ground state is a supersolid, obtained by doping the solids either with particles or vacancies. Recently [13], a complete devil' s staircase has been predicted in the phase diagram of a one-dimensional dipolar Bose gas.

In this work, we also investigate how the previous scenario changes by considering a multi-layer structure. We focus on the simplest situation composed of two 2D layers in which the dipoles are polarized perpendicularly to the planes; the dipolar interaction is then repulsive for particles laying on the same plane, while it is attractive for particles at the same lattice site on different layers. Instead we consider inter-layer tunneling to be suppressed, which makes the system analogous to a bosonic mixture in a 2D lattice. Our calculations show that particles pair into composites, and demonstrate the existence of the novel Pair Super Solid (PSS) quantum phase.

Currently we are studying a 2D lattice where the dipoles are free to point in both directions perpendicularly to the plane, which results in a nearest neighbor repulsive (attractive) interaction for aligned (anti-aligned) dipoles. We find regions of parameters where the ground state is ferromagnetic or antiferromagnetic, and find evidences for the existence of a Counterflow Super Solid (CSS) quantum phase.

Our predictions have direct experimental consequences, and we hope that they will be soon checked in experiments with ultracold dipolar atomic and molecular gases. This thesis is based on the following publications:

- C. Menotti, C. Trefzger, and M. Lewenstein, Metastable States of a Gas of Dipolar Bosons in a 2D Optical Lattice. Physical Review Letters, 98, 235301 (2007).

- C. Trefzger, C. Menotti, and M. Lewenstein, Ultracold dipolar gas in 
an optical lattice: The fate of metastable states. Physical Review A, 78, 043604, (2008).

- C. Trefzger, C. Menotti, and M. Lewenstein, Pair-Supersolid Phase in a Bilayer System of Dipolar Lattice Bosons. Physical Review Letters, 103, 035304, (2009).

- B. Capogrosso-Sansone, C. Trefzger, M. Lewenstein, P. Zoller, and G. Pupillo, Quantum Phases of Cold Polar Molecules in 2D Optical Lattices. arXiv:0906.2009. Accepted for Physical Review Letters publication.

- C. Trefzger, M. Alloing, C. Menotti, F. Dubin, and M. Lewenstein, Counterflow Supersolid of anti-polarized dipolar Bosons in a 2D optical lattice. In preparation. 


\section{Contents}

I Theory and methods 10

1 Dipolar Bose gas in optical lattices $\quad 13$

1.1 Optical lattices . . . . . . . . . . . . . . . . . . 13

1.2 Theory of dilute Bose gases . . . . . . . . . . . . . 15

1.2.1 The Gross-Pitaevskii equation . . . . . . . . . . 17

1.2.2 Bose-Hubbard model . . . . . . . . . . . . . . . . 18

1.3 Dipolar Bose gas . . . . . . . . . . . . . . . . . . . . . . . 22

1.3.1 Properties of the dipole-dipole interaction . . . . . . 22

1.3.2 Polarized dipoles in anisotropic harmonic traps . . . . 23

1.3.3 Mean-field dipolar interaction in a spherical trap . . . . 26

1.3.4 Extended Bose-Hubbard model . . . . . . . . . . . 28

2 Hubbard models: theoretical methods 31

2.1 Superfluid-Mott insulator quantum phase transition in the

Bose-Hubbard model . . . . . . . . . . . . . . . . . . . 31

2.2 The Gutzwiller mean-field approach . . . . . . . . . . . . . 33

2.2.1 Dynamical Gutzwiller approach . . . . . . . . . . . . 34

2.2.2 Perturbative mean-field approach . . . . . . . . . . . 37

2.2.3 Perturbative mean-field vs. dynamical Gutzwiller approach . . . . . . . . . . . . . . . 39

II Metastable states $\quad 41$

3 Dipolar Bosons in a 2D optical lattice 43

3.1 The model . . . . . . . . . . . . . . . . . . . . . 43

3.2 Metastability . . . . . . . . . . . . . . . . 44

3.3 The lifetime . . . . . . . . . . . . . . . . . 47

3.3.1 Parametrization . . . . . . . . . . . . 51

3.3.2 Action and tunneling time . . . . . . . . . . . . 52

3.4 Preparation, manipulation and detection . . . . . . . . 54 
3.4.1 Transfer process . . . . . . . . . . . . . . . 56

3.5 Harmonic confinement . . . . . . . . . . . . . . . . . . 59

4 Conclusions $\quad 61$

III Multiple layers and mixtures 62

5 Dipolar Bosons in a bilayer optical lattice $\quad 65$

5.1 The model . . . . . . . . . . . . . . . . . . 65

5.1.1 Ground state and single-particle single-hole excitations 67

5.2 Low-energy subspace and effective Hamiltonian . . . . . . . 70

5.2.1 Ground state insulating phases and two-particle twohole excitations . . . . . . . . . . . . . 72

5.3 Gutzwiller mean-field approach and validity of the low energy subspace . . . . . . . . . . . . . . . . . 73

6 Counterflow Supersolid of anti-polarized dipolar Bosons in a 2D optical lattice $\quad 78$

6.1 Introduction . . . . . . . . . . . . . . . . . 78

6.2 Hamiltonian of the system . . . . . . . . . . . . . . 78

6.2.1 Filling factor and imbalance . . . . . . . . . . . . . . 79

6.2.2 Low-energy subspace and effective Hamiltonian . . . . 82

6.3 Mean-field . . . . . . . . . . . . . . . . . . . . 85

6.3 .1 Insulating lobes . . . . . . . . . . . . . . . . 86

6.3.2 Counterflow superfluid-supersolid . . . . . . . . . 87

$\begin{array}{lll}7 & \text { Conclusions } & 91\end{array}$

IV Quantum Monte Carlo $\quad 92$

8 Path Integral Monte Carlo and the Worm algorithm 95

8.1 Path Integral Monte Carlo . . . . . . . . . . . . . . . . 95

8.1.1 Path Integral Monte Carlo and the 2D extended Bose-

Hubbard model . . . . . . . . . . . . . . . . . . . 97

8.2 The Worm algorithm . . . . . . . . . . . . . . . . . 100

8.2.1 Updating procedures . . . . . . . . . . . . . . 100

8.2.2 Advantages of the Worm algorithm . . . . . . . . . . 104 
9 Quantum Monte Carlo studies of dipolar gases 106

9.1 Zero momentum Green function and the particle-hole excitations 107

9.2 Incompressible and supersolid phases . . . . . . . . . . . 107

9.2.1 Homogeneous case . . . . . . . . . . . . . . . . 108

9.2 .2 Finite temperature . . . . . . . . . . . . . . 110

9.2.3 Harmonic confinement . . . . . . . . . . . . . 110

10 Conclusions 113

$\begin{array}{ll}\text { A Spectrum of excitations } & 116\end{array}$

$\begin{array}{lr}\text { Bibliography } & 119\end{array}$ 


\section{Part I}

\section{Theory and methods}




\section{Introduction}

The aim of this part of the thesis is twofold. First, to introduce the reader to the basics of the theory of dilute Bose gases of neutral particles, in the presence of an optical lattice, covered by Chapter 1. We start in Sec. 1.1 with the description of an optical lattice: how it is produced in the laboratory and why it can trap neutral particles. After briefly explaining the Gross-Pitaevskii equation in mean-field regime, in Sec. 1.2 we show how such systems realize Bose-Hubbard Hamiltonians (BH), providing the temperature of the gas is low enough to confine the motion of the particles only to the first Bloch band. In Sec. 1.3, we describe the properties of the dipolar interaction: its long-range and anisotropic character, and how it is taken into account in Hubbard type models, therefore realizing the so-called extended BoseHubbard Hamiltonian, which will be the starting point of our theoretical work.

Second, in Chapter 2 we familiarize the reader with the new theoretical tools we have developed during this thesis work, necessary to describe the properties of extended Bose-Hubbard Hamiltonians. First, in Sec. 2.1, we briefly recall that the BH Hamiltonian sustains the superfluid to Mott insulator $(S F-M I)$ quantum phase transition. This is a well known result and we derive it within a mean-field theory $(\mathrm{MF})$. Moreover, we compare the MF predictions with other numerical methods such as Monte Carlo or Density Matrix Renormalization Group calculations, and show that MF results are unsatisfactory for one dimensional systems while they are reasonably good in two and three dimensions. Based on a Gutzwiller Ansatz for the wavefunction of the system, in Sec. 2.2 we make use of the mean-field theory to investigate the ground state properties (and beyond) of extended Bose-Hubbard Hamiltonians. We allow the Gutzwiller amplitudes to be time-dependent, and in Sec. 2.2.1 we derive their dynamical equations, with which it is possible to identify various $M I$ and $S F$ phases of the system through the imaginary time evolution. Due to the presence of the dipolar term in the Hamiltonian, that is responsible for the appearance of many insulating metastable states, it is often challenging to find all the $M I$ phases with the dynamics in imaginary 
time. Therefore, to find all the metastable states compatible with a given range of the dipolar interactions and size of the elementary cell, in Sec. 2.2.2 we derive a perturbative mean-field approach that proves to be more efficient for this purpose. We conclude in Sec. 2.2.3 by comparing the two methods that will be extensively used throughout this thesis. 


\section{Chapter 1}

\section{Dipolar Bose gas in optical lattices}

\section{$1.1 \quad$ Optical lattices}

An optical lattice is an artificial crystal of light, resulting from the interference patterns of two or more counterpropagating laser beams [15]. The wavelengths $\lambda_{i}$ of the laser beams determine the spatial periodicity of the crystal; for example, two lasers of equal wavelengths $\lambda_{x}$ propagating along $x$ but in opposite directions, produce a standing wave with an intensity pattern $I(x)$ which is spatially periodic with periodicity $\lambda_{x} / 2$. An optical lattice can trap neutral atoms by exploiting the energy shifts induced by the radiation on the atomic internal energy levels.

The electric field $\mathbf{E}(\mathbf{r}, t)=2 E_{0} \cos \left(\mathbf{k} \cdot \mathbf{r}-\omega_{L} t\right)$ of a monochromatic laser oscillating with frequency $\omega_{L}$, interacts with a neutral atom, of spatial dimensions much smaller compared to the wavelengths $\lambda_{i}=2 \pi / k_{i},(i=x, y, z)$ of the light, through the Hamiltonian

$$
\hat{H}_{\text {int }}(t)=-\mathbf{d} \cdot \mathbf{E}(\mathbf{r}, t),
$$

where $\mathbf{d}=-e \sum_{i} \mathbf{r}_{i}$ is the electric dipole moment of the atom, $\mathbf{r}_{i}$ the positions of the atomic electrons of charge $e$. With Hamiltonian (1.1), one can easily calculate the energy correction to the ground state of the atom, by means of perturbation theory. The fist order correction vanishes because the dipole operator is odd with respect to space inversion $\left(\mathbf{r}_{i} \rightarrow-\mathbf{r}_{i}\right)$, therefore the first non zero contribution is given by the second order correction

$$
\Delta E(\mathbf{r})=-\frac{1}{2} \alpha\left(\omega_{L}\right)\left\langle\mathbf{E}(\mathbf{r}, t)^{2}\right\rangle_{t}
$$


where

$$
\alpha\left(\omega_{L}\right)=\sum_{\gamma}|\langle\gamma|\mathbf{d} \cdot \hat{\epsilon}| g\rangle|^{2}\left(\frac{1}{E_{\gamma}-E_{g}+\hbar \omega_{L}}+\frac{1}{E_{\gamma}-E_{g}-\hbar \omega_{L}}\right),
$$

is the atomic polarizability [17], and $\langle\cdots\rangle_{t}$ denotes a time average over one oscillation period of the electric field ${ }^{1}$. In the last expression the energies in the denominators are the unperturbed energies of the atom, where $E_{g}$ is its ground state, the sum runs over all excited states and $\hat{\epsilon}$ is the unit vector in the direction of the electric field. In a typical experiment the laser light is far off resonance, which means that the laser frequency is close to one of the unperturbed excited states (e.g $E_{e}=\hbar \omega_{e}$ ), but does not induce any real transition. In such a situation, one can take only the smallest of the denominators (1.3), and the polarizability becomes inversely proportional to the laser detuning from resonance $\hbar \Delta=\hbar \omega_{L}-\left(E_{e}-E_{g}\right)$

$$
\alpha\left(\omega_{L}\right) \simeq-\frac{|\langle e|\mathbf{d} \cdot \hat{\epsilon}| g\rangle|^{2}}{\hbar \Delta}
$$

In this situation, the energy shift is then given by

$$
\Delta E(\mathbf{r})=-\frac{1}{2} \alpha\left(\omega_{L}\right)\left\langle\mathbf{E}(\mathbf{r}, t)^{2}\right\rangle_{t} \propto \frac{I(\mathbf{r})}{\hbar \Delta},
$$

where $I(\mathbf{r})$ is the intensity of the laser. In the dressed atom picture, the energy shift (1.5) is interpreted as an effective potential $V_{\text {opt }}(\mathbf{r})=\Delta E(\mathbf{r})$, that follows the spatial pattern of the laser field intensity, in which the atom moves. In this picture, the atom then feels a force

$$
\mathbf{F}_{\text {dipole }}=-\nabla V_{\text {opt }}(\mathbf{r}),
$$

that attracts it towards the regions of high intensity for the so called reddetuned lasers (i.e. $\Delta<0$ ), while a blue-detuned light (i.e. $\Delta>0$ ) pushes the atom out of the regions of high intensity. In the literature this force is called the dipole force, as it is the resulting interaction of the induced atomic dipole moment with the spatially varying electric field of the light. Note that in order to reduce heating caused by inelastic scattering, i.e. photon absorption and spontaneous emission processes, a large detuning is required because the photon scattering rate scales as $I(\mathbf{r}) / \Delta^{2}$. In the limit of large detuning an optical lattice is therefore non-dissipative, which makes it a basic tool to manipulate cold neutral atoms.

\footnotetext{
${ }^{1}$ more specifically $\langle\cdots\rangle_{t}=\frac{1}{t} \int_{0}^{t} \cdots \mathrm{d} t$ where $t=n \pi / \omega_{L}, n=1,2, \cdots$
} 
For example, the simplest case of a one-dimensional lattice is obtained by the superposition of two lasers propagating in opposite directions, with electric fields linearly polarized, say in the $z$ direction,and given by

$$
\begin{aligned}
E_{z}(x, t) & =2 E_{0} \cos \left(k_{x} x-\omega_{L} t\right)+2 E_{0} \cos \left(-k_{x} x-\omega_{L} t\right) \\
& =4 E_{0} \cos \left(k_{x} x\right) \cos \left(\omega_{L} t\right) .
\end{aligned}
$$

The time average, over one period of oscillation of the electric field, gives then $\left\langle E_{z}(x, t)^{2}\right\rangle_{t}=2 E_{0} \cos ^{2}\left(k_{x} x\right)$ which yields to the spatially varying optical potential

$$
V_{\text {opt }}(x)=V_{0, x} \cos ^{2}\left(k_{x} x\right),
$$

with periodicity $\lambda_{x} / 2=\pi / k_{x}$, and $V_{0, x}=2 E_{0} \alpha\left(\omega_{L}\right)$ from Eq. (1.4). The generalization of a two dimensional (2D) or three dimensional case (3D) is straightforward (see e.g. [17]). For example in figure 1.1 two different geometries are shown.
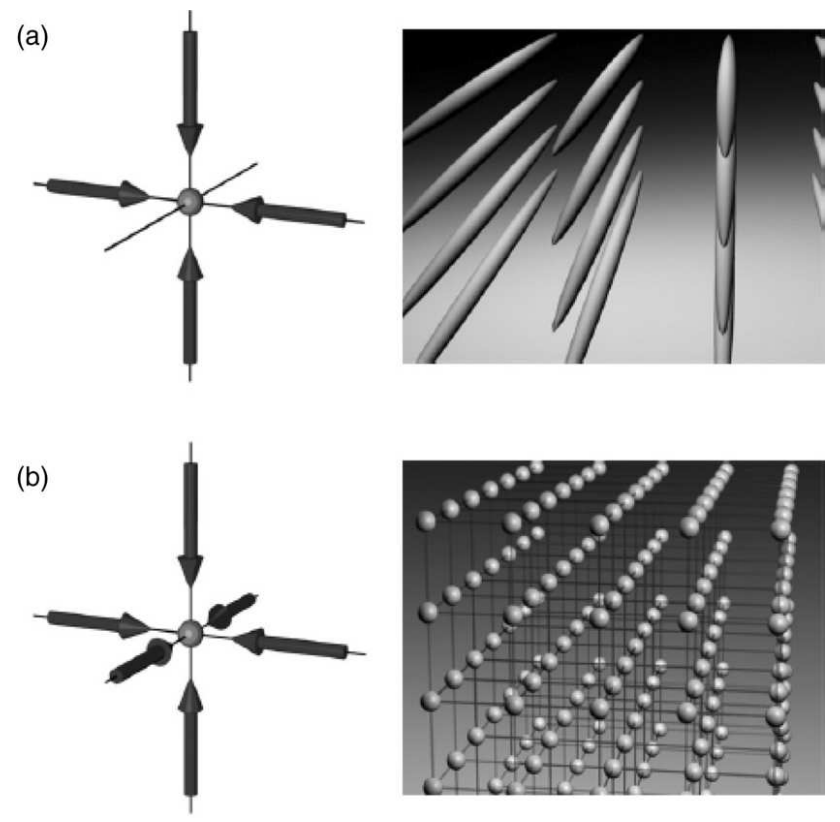

Figure 1.1: Picture of an optical potential. (a) 2D square lattice of quasi 1D traps; (b) a 3D cubic lattice, picture taken from [15].

\subsection{Theory of dilute Bose gases}

In this section we recall some basic theory of a dilute gas of neutral bosonic particles, at temperature $T$ well below the degeneracy temperature. At these 
temperatures the gas is a Bose Einstein condensate (BEC). The type of particles we consider here can be atoms or molecules.

For a dilute gas, the interparticle separation (typically of the order of $10^{2}$ $\mathrm{nm}$ for alkali atoms [17]) is an order of magnitude larger than the length scales associated with the atom-atom interaction. In other words, a dilute gas of density $n$ is a very rarefied gas in which the "spatial extension" of an atom is much smaller than the average volume per particle $n^{-1}$. Because of this condition, the two-body interaction dominates the physics while threebody or more are very unlikely and essentially not important. The two-body interatomic potential $V(\mathbf{r})$ depends on the type of particles one consider, the relative distance between the atoms $\mathbf{r}=\mathbf{r}_{1}-\mathbf{r}_{2}$ and on their internal states. For alkali atoms, the potential is strongly repulsive for small atomic separations while for large atomic distances it is dominated by the van der Waals attractions that decay as $-C_{6} / \mathbf{r}^{6}$, where the coefficient $C_{6}$ depends on the atomic species.

Here we will consider only elastic scattering, where the internal states of the two atoms do not change in the collision process. If the temperature of the gas is very low, i.e. $T \rightarrow 0$, then the kinetic energy of the particles is very small compared to the centrifugal barrier and only $s$-wave scattering takes place. Therefore, the only important parameter is the scattering length given by

$$
a_{s}=\frac{m}{4 \pi \hbar^{2}} \int \mathrm{d}^{3} r V(\mathbf{r})
$$

with $m$ being the mass of the atoms. This quantity has the dimensions of a length and has the physical interpretation of the radius the atoms would have if they were considered to be perfect billiard balls. The condition for the diluteness of the gas then reads

$$
n a_{s}^{3} \ll 1
$$

where $n$ is the density of the gas and $n a_{s}^{3}$ is called the gas parameter. One can invert the expression (1.9) and think of an effective interaction between the two particles proportional to the scattering length, and given by

$$
V_{\text {eff }}(\mathbf{r})=g \delta^{(3)}(\mathbf{r})
$$

where $g$ is defined as

$$
g=\frac{4 \pi \hbar^{2} a_{s}}{m}
$$

and $\delta$ is the Dirac delta function so that the particles are considered to be point-like. Note also that since the effective interaction depends only on the scattering length, it is repulsive (attractive) for positive (negative) $a$, and it 
can be dynamically modified for example in alkali atoms just by varying an external magnetic field near a Feshbach resonance.

\subsubsection{The Gross-Pitaevskii equation}

The quantum state of a gas of $N$ particles is described by the many-body wavefunction $\Psi\left(\mathbf{r}_{1}, \mathbf{r}_{2}, \ldots, \mathbf{r}_{\mathrm{N}}\right)$, and the time evolution of the system is determined by the Schrödinger equation. In a BEC, one can describe the dynamics of the condensate just through the Gross-Pitaevskii (GP) equation $[16,17]$ given by

$$
i \hbar \frac{\partial}{\partial t} \Psi_{0}(\mathbf{r}, t)=\left(-\frac{\hbar^{2} \nabla^{2}}{2 m}+V_{\text {ext }}(\mathbf{r})+g\left|\Psi_{0}(\mathbf{r}, t)\right|^{2}\right) \Psi_{0}(\mathbf{r}, t),
$$

where $\Psi_{0}(\mathbf{r}, t)$ is the BEC wavefunction, also called the order parameter. The interaction between particles has been taken into account in a meanfield approximation by the term $g\left|\Psi_{0}(\mathbf{r}, t)\right|^{2}$, and $g$ is given in Eq. 1.12. The two-body effective potential is given by Eq. (1.11), $V_{\text {ext }}(\mathbf{r})$ is an external trapping potential, and the order parameter is normalized to the total number of particles, i.e. $N=\int \mathrm{d}^{3} r\left|\Psi_{0}(\mathbf{r}, t)\right|^{2}$. Equation (1.13) was independently derived by Gross and Pitaevskii in 1961, it is one of the main theoretical tools for investigating dilute weakly interacting Bose gases at low temperatures, and it has the typical form of a mean field equation where the order parameter must be calculated in a self-consistent way.

The GP equation has proven to be a very useful tool to describe the physics of weakly interacting Bose-Einstein atomic condensates in the early ages of this field. With this formalisms, and its extension to include small fluctuations given by Bogoliubov theory, one can describe accurately, among others, the collective excitations of the systems, the response to rotations including the formation of vortices, the propagation of sound, the presence of dynamical instabilities. Generally speaking, the GP treatment is well suited in the regime of full coherence, when a single macroscopically occupied matterwave correctly describes the system. At the end of the '90, few years after the creation of the first alkali BECs in the lab, the need of "going beyond GP" started to be very strongly felt, due to the theoretical interest and experimental possibility of going into the strongly correlated regime. In fact, the presence of strong interactions, strong rotations and/or special trapping potentials can limit the validity of the GP equation. For instance a strong confinement in one or two dimensions can reduce the system to an effectively $2 \mathrm{D}$ or $1 \mathrm{D}$ one. A strong rotation combined with interactions can lead to quantum Hall physics. Also the presence of a deep optical lattices, 
when the combined effect of interactions and trapping potential leads to a "fragmentation" of the condensate, requires more sophisticated descriptions.

In this thesis we are interested in describing the physics of Bosons trapped in a periodic optical potential $\left(V_{\text {opt }}\right)$ and eventually also confined in a magnetic harmonic trap $\left(V_{\text {ho }}\right)$, the total external field being given by the sum

$$
V_{\text {ext }}(\mathbf{r})=V_{\mathrm{opt}}(\mathbf{r})+V_{\mathrm{ho}}(\mathbf{r})=\sum_{i=x, y, z} V_{0, i} \cos ^{2}\left(k_{i} r_{i}\right)+\frac{1}{2} m \sum_{i=x, y, z} \omega_{\mathrm{i}}^{2} r_{i}^{2},
$$

where $\left(V_{0, x}, V_{0, y}, V_{0, z}\right)$ is the depth of the optical lattice in the three spatial directions and $\left(\omega_{\mathrm{x}}, \omega_{\mathrm{y}}, \omega_{\mathrm{z}}\right)$ the frequencies of the harmonic trap. In order to describe the physics of Bosons trapped in the potential (1.14), we need to "go beyond" the GP equation, and we will devote the following sections to this purpose.

\subsubsection{Bose-Hubbard model}

The starting point of our discussion is Hamiltonian (1.15), written in the second quantization formalism in terms of the creation and annihilation operators for Bosons, $\hat{\psi}^{\dagger}(\mathbf{r})$ and $\hat{\psi}(\mathbf{r})$ respectively, and given by the expression

$$
\hat{H}=\int \mathrm{d}^{3} r \hat{\psi}^{\dagger}(\mathbf{r})\left[-\frac{\hbar^{2} \nabla^{2}}{2 m}+V_{\text {ext }}(\mathbf{r})+\frac{g}{2} \hat{\psi}^{\dagger}(\mathbf{r}) \hat{\psi}(\mathbf{r})-\mu\right] \hat{\psi}(\mathbf{r}),
$$

where the first term in square brackets is the kinetic energy, $V_{\text {ext }}(\mathbf{r})=$ $V_{\text {opt }}(\mathbf{r})+V_{\text {ho }}(\mathbf{r})$ is the external trapping potential (1.14) and we have used the simplified contact interaction (1.11). We work in the grand canonical ensemble such that the chemical potential $\mu$ fixes the total number of particles. Additionally, we assume the harmonic confinement to change on a scale larger than the one of the optical lattice, such that we can consider the effect of the magnetic trapping to be constant over a single site of the lattice.

In this formalism, the field operators can be written in the basis of singleparticle wave functions $\left\{\Phi_{n}(\mathbf{r})\right\}_{n}$, where $n$ is a complete set of single particle quantum numbers

$$
\begin{aligned}
\hat{\psi}(\mathbf{r}) & =\sum_{n} \Phi_{n}(\mathbf{r}) \hat{a}_{n} \\
\hat{\psi}^{\dagger}(\mathbf{r}) & =\sum_{n} \Phi_{n}^{*}(\mathbf{r}) \hat{a}_{n}^{\dagger}
\end{aligned}
$$

with $\hat{a}_{n}^{\dagger}$ and $\hat{a}_{n}$ being the creation and annihilation operators on the Fock state for the mode $n$, i.e. $\hat{a}_{n}^{\dagger}|n\rangle=\sqrt{n+1}|n+1\rangle$ and $\hat{a}_{n}|n\rangle=\sqrt{n}|n-1\rangle$. 
Also, the field operators satisfy the usual commutation relations for Bosons

$$
\begin{aligned}
{\left[\hat{\psi}(\mathbf{r}), \hat{\psi}^{\dagger}\left(\mathbf{r}^{\prime}\right)\right] } & =\sum_{n=0}^{\infty} \Phi_{n}(\mathbf{r}) \Phi_{n}^{*}\left(\mathbf{r}^{\prime}\right)=\delta^{3}\left(\mathbf{r}-\mathbf{r}^{\prime}\right), \\
{\left[\hat{\psi}(\mathbf{r}), \hat{\psi}\left(\mathbf{r}^{\prime}\right)\right] } & =\left[\hat{\psi}^{\dagger}(\mathbf{r}), \hat{\psi}^{\dagger}\left(\mathbf{r}^{\prime}\right)\right]=0 .
\end{aligned}
$$

It is well known [20], that the spectrum of a single particle in a periodic potential is characterized by bands of allowed energies and energy gaps, and the single particle wave functions are described by Bloch functions $\Phi_{\alpha \mathbf{k}}(\mathbf{r})$ with band index $\alpha$ and quasi-momentum $\hbar \mathbf{k}$. Alternatively, there exists a complementary single-particle basis given by the Wannier functions [20, 21] $w_{\alpha}\left(\mathbf{r}-\mathbf{R}_{i}\right)$, where $\mathbf{R}_{i}$ is a lattice vector pointing at site $i$ and $w_{\alpha}(\mathbf{r})$ are defined as the Fourier transform of Bloch functions

$$
w_{\alpha}(\mathbf{r})=\frac{1}{\sqrt{N_{S}}} \sum_{\mathbf{k}} e^{-i \mathbf{k} \cdot \mathbf{r}} \Phi_{\alpha \mathbf{k}}(\mathbf{r}),
$$

where $N_{S}$, is the total number of sites in the lattice. The Wannier functions form a complete orthonormal set, so one may write the field operators (1.16) as

$$
\begin{aligned}
\hat{\psi}(\mathbf{r}) & =\sum_{\alpha \mathbf{k}} \Phi_{\alpha \mathbf{k}}(\mathbf{r}) \hat{a}_{\alpha \mathbf{k}}=\sum_{\alpha, i} w_{\alpha}\left(\mathbf{r}-\mathbf{R}_{i}\right) \hat{a}_{\alpha, i} \\
\hat{\psi}^{\dagger}(\mathbf{r}) & =\sum_{\alpha \mathbf{k}} \Phi_{\alpha \mathbf{k}}^{*}(\mathbf{r}) \hat{a}_{\alpha \mathbf{k}}^{\dagger}=\sum_{\alpha, i} w_{\alpha}^{*}\left(\mathbf{r}-\mathbf{R}_{i}\right) \hat{a}_{\alpha, i}^{\dagger}
\end{aligned}
$$

Wannier functions are useful in the case of deep optical lattices where tight binding approximation apply. The big advantage of using Wannier functions $w_{\alpha}\left(\mathbf{r}-\mathbf{R}_{i}\right)$ is that they are localized and centered around the lattice site pointed by $\mathbf{R}_{i}$.

If the temperature of the system is low enough, and the interactions between the particles is not sufficient to induce transitions between the bands, one may restrict only to the first Bloch band because the particles have insufficient energy to overcome the gap that separates the first band from the others. This amounts to keep in (1.19) only the lowest of the $\alpha$ indices, which we omit for simplicity of notation and therefore the Hamiltonian (1.15) becomes

$$
\hat{H}=-\sum_{i, j} J_{i j} \hat{a}_{i}^{\dagger} \hat{a}_{j}+\sum_{i, j, k, l} \frac{U_{i, j, k, l}}{2} \hat{a}_{i}^{\dagger} \hat{a}_{j}^{\dagger} \hat{a}_{k} \hat{a}_{l}-\sum_{i, j} \mu_{i, j} \hat{a}_{i}^{\dagger} \hat{a}_{j} .
$$


The quantities in the sums are given by

$$
\begin{aligned}
J_{i j} & =-\int \mathrm{d}^{3} r w^{*}\left(\mathbf{r}-\mathbf{R}_{i}\right)\left[-\frac{\hbar^{2} \nabla^{2}}{2 m}+V_{\mathrm{opt}}(\mathbf{r})\right] w\left(\mathbf{r}-\mathbf{R}_{j}\right) \\
U_{i, j, k, l} & =g \int \mathrm{d}^{3} r w^{*}\left(\mathbf{r}-\mathbf{R}_{i}\right) w^{*}\left(\mathbf{r}-\mathbf{R}_{j}\right) w\left(\mathbf{r}-\mathbf{R}_{k}\right) w\left(\mathbf{r}-\mathbf{R}_{l}\right) \\
\mu_{i, j} & =\int \mathrm{d}^{3} x w^{*}\left(\mathbf{r}-\mathbf{R}_{i}\right)\left[\mu-V_{\mathrm{ho}}(\mathbf{r})\right] w\left(\mathbf{r}-\mathbf{R}_{j}\right) .
\end{aligned}
$$

The Wannier functions are localized on the lattice sites, the deeper the lattice the more localized they are. For a sufficiently deep optical potential, then in Eq. (1.22) and (1.23) the dominant contributions are given by $U_{i, i, i, i}$ and $\mu_{i, i}$. For the kinetic part (1.21), there is a constant contribution given by $J_{i, i}$ and due to the presence of the derivative in the integration, there is also a positive matrix element for nearest neighboring sites $J_{i, j}>0$. The two situations are qualitatively shown in Fig. (1.2) where we have approximated the Wannier functions with two Gaussians respectively localized at site $i$ and $j$ of the lattice. However, we stress that the picture provided by Gaussian functions is only qualitative. In fact, in order to be quantitatively correct, one needs to calculate the proper matrix elements with Wannier functions.
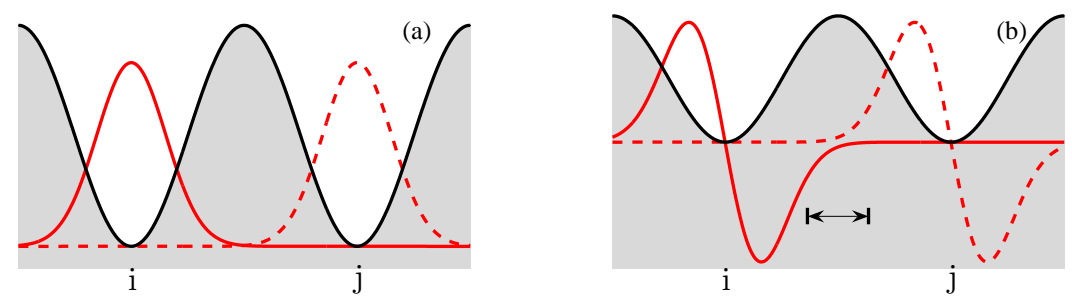

Figure 1.2: (a) Two Gaussians localized on neighboring sites $i$ and $j$ of an optical lattice having negligible overlap. (b) The first derivative of the Gaussian functions instead, show a negative overlap in the region indicated by the arrow, which leads to a positive matrix element $J_{i, j}>0$.

With the above considerations, we can now write the celebrated Bose-Hubbard Hamiltonian in the form

$$
\hat{H}_{\mathrm{BH}}=-J \sum_{\langle i j\rangle} \hat{a}_{i}^{\dagger} \hat{a}_{j}+\frac{U}{2} \sum_{i} \hat{n}_{i}\left(\hat{n}_{i}-1\right)-\sum_{i} \mu_{i} \hat{n}_{i},
$$

where $\langle i j\rangle$ indicates sum over nearest neighbors, the tunneling coefficient $J=J_{i, j}=J_{j, i}$ for hermiticity, the on-site interaction $U=g \int \mathrm{d}^{3} r|w(\mathbf{r})|^{4}$, 
$\hat{n}_{i}=\hat{a}_{i}^{\dagger} \hat{a}_{i}$ is the number operator at site $i$, and we have neglected $J_{i, i}$ since it gives a constant contribution for each site. The harmonic confinement, since it is assumed to be constant across one lattice site, has been taken into account in the chemical potential as

$$
\mu_{i}=\mu-\frac{1}{2} m \vec{\omega}^{2} \cdot\left(\mathbf{R}_{i}-\mathbf{R}_{\mathbf{0}}\right)^{2},
$$

where $\mathbf{R}_{\mathbf{0}}$ is the center of the harmonic trap with frequencies given by $\vec{\omega}=$ $\left(\omega_{\mathrm{x}}, \omega_{\mathrm{y}}, \omega_{\mathrm{z}}\right)$ in the three directions. The second term on the right hand side of Eq. (1.25) is practically a chemical potential that differs from site to site and it is often called the local chemical potential.

For a one dimensional optical lattice $V_{\text {opt }}(x)=V_{0} \sin ^{2}(k x)$ with wavevector $k=2 \pi / \lambda$, Fig. 1.3 shows both the on-site interaction $U$ (solid line) and the tunneling coefficient $J$ (dashed line) as a function of the optical lattice depth $V_{0}$, where all the quantities are measured in terms of the recoil energy $E_{R}=\hbar^{2} k^{2} / 2 m$, that is the energy acquired by the atom after absorbing a photon with momentum $\hbar k$. The lattice parameters $U$ and $J$ were calculated numerically in e.g. [22] for different values of $V_{0}$. From Fig. 1.3 (b), it is clear that it is possible to change the tunneling coefficient $J$ over a wide range, going from a situation of practically isolated lattice sites at $V_{0}=25 E_{R}$ up to a regime in which particles can tunnel from site to site at $V_{0}=5 E_{R}$, only by changing the optical potential depth by a few tens of recoil energies, and leaving the on-site interaction $U$ practically unchanged.
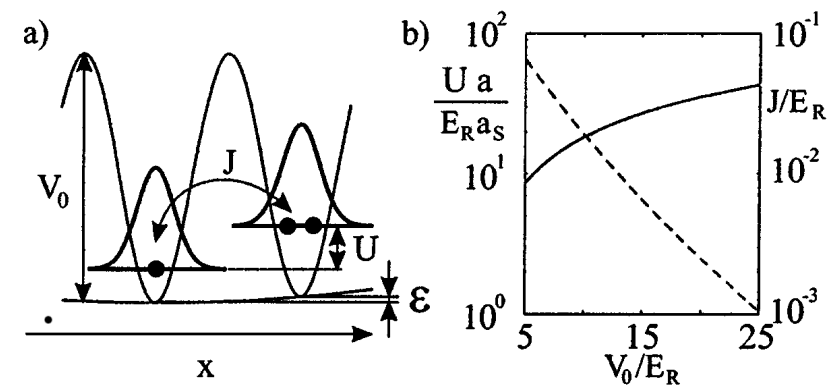

Figure 1.3: (a) Schematic representation of a 1D optical lattice; (b) scaled on-site $U$ (solid line) and tunneling coefficient $J$ (dashed line) dependence on the optical potential depth $V_{0}$. The on-site interaction is multiplied by $a / a_{s}(\gg 1)$, where $a=\lambda / 2$ is the lattice period and $a_{s}$ is the s-wave scattering length for atoms of equal mass $m$. Figure from [22]. 


\subsection{Dipolar Bose gas}

\subsubsection{Properties of the dipole-dipole interaction}

Two particles 1 and 2 in a three dimensional space, at relative distance $\mathbf{r}$ and with dipole moments along the unit vectors $\mathbf{e}_{\mathbf{1}}$ and $\mathbf{e}_{\mathbf{2}}$ as in Fig. 1.4 (a), interact through the dipole-dipole interaction such that their interaction energy is given by

$$
U_{\mathrm{dd}}(\mathbf{r})=\frac{C_{\mathrm{dd}}}{4 \pi} \frac{\left(\mathbf{e}_{1} \cdot \mathbf{e}_{2}\right) r^{2}-3\left(\mathbf{e}_{1} \cdot \mathbf{r}\right)\left(\mathbf{e}_{2} \cdot \mathbf{r}\right)}{r^{5}},
$$

where $r=|\mathbf{r}|$, and $U_{\mathrm{dd}}(\mathbf{r})=U_{\mathrm{dd}}(-\mathbf{r})$. The dipolar coupling constant $C_{\mathrm{dd}}$ is different for particles having a permanent magnetic dipole moment $\mu$, and for particles having a permanent electric dipole moment $d$, and is respectively given by

$$
C_{\mathrm{dd}}= \begin{cases}\mu_{0} \mu^{2} & \text { magnetic } \\ d^{2} / \varepsilon_{0} & \text { electric }\end{cases}
$$

where $\mu_{0}$ is the vacuum permeability, and $\varepsilon_{0}$ is the vacuum permittivity.

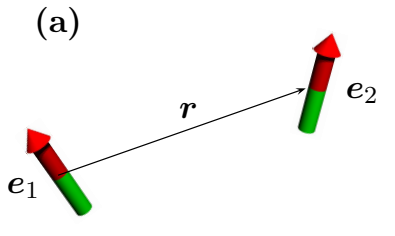

(c)

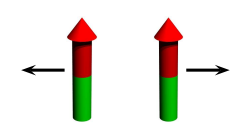

(b)

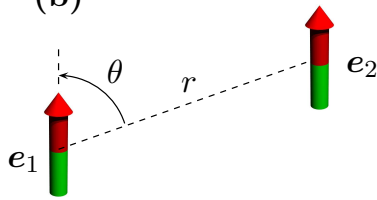

(d)

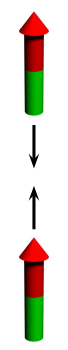

Figure 1.4: (a) Two dipoles, 1 and 2, directed along unit vectors $\mathbf{e}_{1}$ and $\mathbf{e}_{2}$ and separated by a distance $r$. (b) Polarized dipoles, for which the interaction depends on the angle $\theta$ between the direction of the dipoles and the interparticle separation $r$. This results in a repulsive interaction for $\theta=\pi / 2$ (c), and attractive for $\theta=0(\pi)(\mathrm{d})$. Figure from [40]. 
The dipole-dipole interaction (1.26) has a long-range character; this is because at large distances it decays as $U_{\mathrm{dd}} \sim 1 / r^{3}$, contrary to the typical van der Waals potential that behaves like $U_{\mathrm{vdW}} \sim-1 / r^{6}$. Also, from (1.26) it is easy to see the anisotropic property of this interaction; for polarized atoms, i.e. all dipoles pointing in the same direction (say $z$ ), the interaction reduces to

$$
U_{\mathrm{dd}}(\mathbf{r})=\frac{C_{\mathrm{dd}}}{4 \pi} \frac{1-3 \cos ^{2} \theta}{r^{3}},
$$

where $\theta$ is the angle between the dipole and the relative distance of the particles, as in Fig 1.4 (b). The interaction is repulsive for $\theta=\pi / 2$ as the example of Fig 1.4 (c), and attractive for $\theta=0$ as shown in Fig $1.4(\mathrm{~d})$. The situation is reversed for anti-parallel dipoles, where a minus sign appears in front of Eq. (1.28), and therefore the interaction is attractive for $\theta=\pi / 2$ while $\theta=0$ gives rise to repulsion.

The scattering properties of ultracold atoms, in the simple case of isotropic van der Waals interactions, are entirely described by the s-wave scattering length and the potential can be replaced by the effective contact interaction (1.11). In the presence of a dipolar interaction as (1.26), because of its long range (decay as $1 / r^{3}$ ) and anisotropic character (strong dependence on the relative angles between the dipoles), all partial waves contribute to the scattering problem and also partial waves with different angular momenta couple with each other. While for Fermions, replacing the real potential (1.26) with an effective dipolar interaction as (1.11) is reasonable [23], for Bosons this it is not obvious, and in recent years it has been the subject of intensive studies $[24,25,26,27]$. In the presence of an optical lattice, it has been recently argued [41] that in a 1D geometry, replacing the real dipolar potential with an effective interaction as (1.11) is reasonable as long as the optical lattice is shallow enough. However, in the most general case it is necessary to account for the full expression of the dipole-dipole interaction potential (1.26).

\subsubsection{Polarized dipoles in anisotropic harmonic traps}

We now move to the description of a BEC of polarized dipoles, pointing along the $z$ axis. For polarized dipolar BECs, due to the anisotropy of the dipolar interactions, the geometry of the trapping potential plays a fundamental role, first in determining the spatial distribution of the density, and second in the stability of the gas.

Qualitatively, there are two extreme scenarios depending on the shape of the confining potential, shown in Fig. 1.5: (i) for a cigar-shaped trap elongated along the $z$ axis, i.e. with an aspect ratio between the axial $\omega_{z}$ and radial frequencies $\omega_{\rho}=\omega_{x}=\omega_{y}$ given by $\lambda=\omega_{z} / \omega_{\rho} \ll 1$, the density is 
mainly distributed along the polarization axis and the effect of dipole-dipole interaction is mostly attractive, which might lead to an instability of the gas even in the presence of a weak repulsive contact interaction; (ii), for a pancake-shaped trap, which is strongly confining along the $z$ axis, i.e. $\lambda \gg 1$, the dipolar interaction is mostly repulsive and the BEC is always stable for repulsive contact interactions and might be stable even for attractive contact interactions. In an intermediate situation in which the confining potential is perfectly spherical, the density distribution is then isotropic and the dipoledipole interaction averages out to zero, which leads to a stable BEC for repulsive contact interactions. One can switch between one or the other scenario, just by adjusting the frequency of the confining potential along the $z$ axis with respect to the axial $x$ and $y$, and therefore it is natural to expect that for any given $\lambda$ there is a critical value for the scattering length $a_{\text {crit }}$ below which the BEC is unstable [43].

(a)

(b)

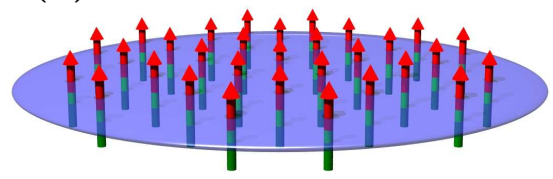

Figure 1.5: Polarized dipoles in anisotropic harmonic potentials. (a) in a cigar shaped trap elongated in the direction of polarization, the resulting dipolar interaction is attractive, and (b) in a pancake trap with a strong confinement in the direction of polarization, the dipolar interactions are repulsive. Figure taken from [40].

One can quantitatively describe the above scenarios starting from the Hamiltonian of the system, which in the presence of the dipole-dipole interaction (1.28) reads

$$
\begin{aligned}
\hat{H} & =\int \mathrm{d}^{3} r \hat{\psi}^{\dagger}(\mathbf{r})\left[-\frac{\hbar^{2} \nabla^{2}}{2 m}+V_{\text {ext }}(\mathbf{r})+\frac{g}{2} \hat{\psi}^{\dagger}(\mathbf{r}) \hat{\psi}(\mathbf{r})-\mu\right] \hat{\psi}(\mathbf{r}) \\
& +\frac{1}{2} \iint \mathrm{d}^{3} r_{1} \mathrm{~d}^{3} r_{2} \hat{\psi}^{\dagger}\left(\mathbf{r}_{1}\right) \hat{\psi}^{\dagger}\left(\mathbf{r}_{2}\right) U_{\mathrm{dd}}\left(\mathbf{r}_{1}-\mathbf{r}_{2}\right) \hat{\psi}\left(\mathbf{r}_{1}\right) \hat{\psi}\left(\mathbf{r}_{2}\right)
\end{aligned}
$$

With the same approximations used to derive the Gross-Pitaevskii equation, one can write the Boson field operator $\hat{\psi}(\mathbf{r})=\Psi_{0}(\mathbf{r})+\delta \hat{\psi}(\mathbf{r})$ as a sum of a classical field $\Psi_{0}(\mathbf{r})$, the condensate wave function, plus the non condensate component $\delta \hat{\psi}(\mathbf{r})[16]$. By neglecting the fluctuations $\delta \hat{\psi}(\mathbf{r})$, one can calculate 
the energy of the BEC given by

$$
\begin{aligned}
E[\psi]= & \int\left[-\frac{\hbar^{2}}{2 m}|\nabla \psi(\mathbf{r})|^{2}+V_{\text {ext }}(\mathbf{r})|\psi(\mathbf{r})|^{2}+\frac{g}{2}|\psi(\mathbf{r})|^{4}\right. \\
& \left.+\frac{1}{2}|\psi(\mathbf{r})|^{2} \int U_{\mathrm{dd}}\left(\mathbf{r}-\mathbf{r}^{\prime}\right)\left|\psi\left(\mathbf{r}^{\prime}\right)\right|^{2} \mathrm{~d}^{3} r^{\prime}\right] \mathrm{d}^{3} r .
\end{aligned}
$$

Within a variational Ansatz, we assume the condensate wave function to be a Gaussian of axial width $\sigma_{z}$ and radial width $\sigma_{x}=\sigma_{y}=\sigma_{\rho}$, normalized to the total number of particles $N$, namely

$$
\Psi_{0}(z, \rho)=\sqrt{\frac{N}{\pi^{3 / 2} \sigma_{\rho}^{2} \sigma_{z} a_{\mathrm{ho}}^{3}}} \exp \left[-\frac{1}{2 a_{\mathrm{ho}}^{2}}\left(\frac{\rho^{2}}{\sigma_{\rho}^{2}}+\frac{z^{2}}{\sigma_{z}^{2}}\right)\right],
$$

where $a_{\mathrm{ho}}=\sqrt{\hbar /(m \bar{\omega})}$ is the harmonic oscillator length with average trap frequency $\bar{\omega}=\left(\omega_{\rho}^{2} \omega_{z}\right)^{1 / 3}$. Therefore, inserting the Ansatz (1.31) into the energy functional Eq. (1.30), after integration we find the energy of the BEC to be a function of the widths of the Gaussians, namely

$$
E_{0}\left(\sigma_{z}, \sigma_{\rho}\right)=E_{\text {kin }}+E_{\text {trap }}+E_{\text {contact }}+E_{\mathrm{dd}}
$$

with the kinetic energy

$$
E_{\mathrm{kin}}=\frac{N \hbar \bar{\omega}}{4}\left(\frac{2}{\sigma_{\rho}^{2}}+\frac{1}{\sigma_{z}^{2}}\right)
$$

the potential energy due to the trap

$$
E_{\text {trap }}=\frac{N \hbar \bar{\omega}}{4 \lambda^{2 / 3}}\left(2 \sigma_{\rho}^{2}+\lambda^{2} \sigma_{z}^{2}\right)
$$

the contact interaction energy given by

$$
E_{\text {contact }}=\frac{\hbar \bar{\omega}}{\sqrt{2 \pi} a_{\mathrm{ho}}} \frac{1}{\sigma_{\rho}^{2} \sigma_{z}} a_{s}
$$

and the contribution coming from the dipolar term

$$
E_{\mathrm{dd}}=-\frac{\hbar \bar{\omega} a_{\mathrm{dd}}}{\sqrt{2 \pi} a_{\mathrm{ho}}} \frac{1}{\sigma_{\rho}^{2} \sigma_{z}} f(\kappa)
$$

where we have introduced the dipolar length $a_{\mathrm{dd}}=\frac{C_{\mathrm{dd}} m}{12 \pi \hbar^{2}}$, with $C_{\mathrm{dd}}$ given in Eq. (1.27), which measures the absolute strength of the dipolar interaction, 
$\kappa=\sigma_{\rho} / \sigma_{z}$ is the aspect ratio of the density distribution, and the function $f$ is given by

$$
f(\kappa)=\frac{1+2 \kappa^{2}}{1-\kappa^{2}}-\frac{3 \kappa^{2} \operatorname{artanh} \sqrt{1-\kappa^{2}}}{\left(1-\kappa^{2}\right)^{3 / 2}} .
$$

While the integrals needed to obtain $(1.33,1.34,1.35)$ are easy to calculate since they contain only Gaussian functions and their derivatives, the integral to get (1.36) is not straightforward due to the presence of the dipolar potential $U_{\mathrm{dd}}\left(\mathbf{r}_{1}-\mathbf{r}_{2}\right)$. See section 1.3.3 for more details. In the left panel of Fig. 1.6, we show the behavior of the function $f(\kappa)$ as $\kappa$ is continuously varied from $\kappa=10^{-2}$ to $\kappa=10^{2}$. The function takes the asymptotic values of $f(0)=1$, $f(\infty)=-2$, and it vanishes for $\kappa=1$, which implies that for a spherical density distribution the dipole-dipole mean-field interaction (1.36) averages out to zero. Therefore we notice that it is possible to control the strength and the sign of the mean-field dipolar interaction just by adjusting the aspect ratio $\lambda$ between the axial and the radial frequencies of the confining trap. The

total interaction energy is provided by the sum of the contact (1.35) plus the dipolar interaction energy (1.36), given by

$$
E_{\mathrm{int}}=\frac{\hbar \bar{\omega} a_{\mathrm{dd}}}{\sqrt{2 \pi} a_{\mathrm{ho}}} \frac{1}{\sigma_{\rho}^{2} \sigma_{z}}\left(\frac{a_{s}}{a_{\mathrm{dd}}}-f(\kappa)\right) .
$$

The stability of the gas requires a repulsive interaction $E_{\text {int }}>0$, which leads to the condition

$$
\frac{a_{s}}{a_{\mathrm{dd}}}-f(\kappa)>0
$$

and can be adjusted ad-hoc by changing the frequencies of the trap in the three directions.

To determine the stability threshold $a_{\text {crit }}(\lambda)$, one needs to minimize the energy (1.32) with respect to the variational parameters $\sigma_{\rho}$ and $\sigma_{z}$ for fixed values of $N, \lambda$ and $\bar{\omega}$. The results are summarized in the right panel of Fig. 1.6 as a thin line, while the thick line represents more accurate results calculated from solving numerically the Gross-Pitaevskii equation [42]. The dots with error bars correspond to experimental data taken from [45].

\subsubsection{Mean-field dipolar interaction in a spherical trap}

In order to calculate the mean-field dipolar interaction energy (1.36), we insert the Gaussian Ansatz (1.31) into the the second of the integrals (1.30), and we get to the expression

$$
E_{\mathrm{dd}}=\frac{1}{2} \iint \mathrm{d}^{3} r_{1} \mathrm{~d}^{3} r_{2} \rho\left(\mathbf{r}_{1}\right) \rho\left(\mathbf{r}_{2}\right) U_{\mathrm{dd}}\left(\mathbf{r}_{1}-\mathbf{r}_{2}\right),
$$



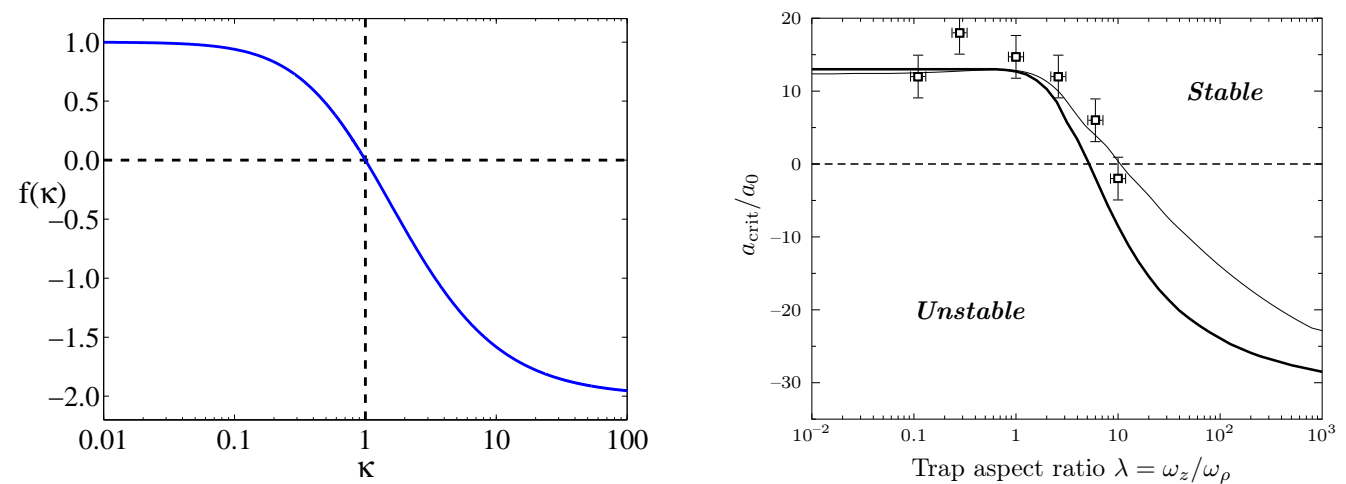

Figure 1.6: (Left panel), $\kappa$ dependence of the $f(\kappa)$ function that appears in the mean-field dipolar interaction. (Right panel) Stability diagram of a dipolar condensate: the thin line is the solution for $a_{\text {crit }}(\lambda) / a_{0}$ calculated with the Gaussian Ansatz (1.31), where $a_{0}$ is the s-wave scattering length, while the thick line is the numerical solution of the GP equation [42]. The dots with error bars are experimental data [45]. Figure taken from [40].

with $\rho(\mathbf{r})=\left|\Psi_{0}(\mathbf{r})\right|^{2}$ being the condensate density at $\mathbf{r}$. The last integral can be simplified by means of convolution theorem $[43,44]$ which states

$$
\int \mathrm{d}^{3} r_{2} U_{\mathrm{dd}}\left(\mathbf{r}_{1}-\mathbf{r}_{2}\right) \rho\left(\mathbf{r}_{2}\right)=\mathcal{F}^{-1}\left\{\widetilde{U_{\mathrm{dd}}}(\mathbf{k}) \widetilde{\rho}(\mathbf{k})\right\},
$$

where $\widetilde{U_{\mathrm{dd}}}(\mathbf{k})$ and $\widetilde{\rho}(\mathbf{k})$ are the Fourier transform respectively of the dipoledipole potential and the density. $\mathcal{F}^{-1}$ indicates the inverse Fourier transform, and using its definition we can write

$$
\begin{aligned}
E_{\mathrm{dd}} & =\frac{1}{2} \int \mathrm{d}^{3} r_{1} \rho\left(\mathbf{r}_{1}\right) \frac{1}{(2 \pi)^{3}} \int \mathrm{d}^{3} k \widetilde{U_{\mathrm{dd}}}(\mathbf{k}) \widetilde{\rho}(\mathbf{k}) e^{i \mathbf{k} \cdot \mathbf{r}_{1}} \\
& =\frac{1}{2(2 \pi)^{3}} \int \mathrm{d}^{3} k \widetilde{U_{\mathrm{dd}}}(\mathbf{k}) \widetilde{\rho}^{2}(\mathbf{k}),
\end{aligned}
$$

where in the last passage we have used the relation $\widetilde{\rho}(\mathbf{k})=\widetilde{\rho}(-\mathbf{k})$.

The Fourier transform of the dipole-dipole interaction (1.28) is given by

$$
\widetilde{U_{\mathrm{dd}}}(\mathbf{k})=\int \mathrm{d}^{3} r U_{\mathrm{dd}}(\mathbf{r}) e^{-i \mathbf{k r}}=C_{\mathrm{dd}}\left(\cos ^{2} \gamma-1 / 3\right),
$$

where $\gamma$ is the angle between $\mathbf{k}$ and the polarization direction, and $C_{\mathrm{dd}}$ is given by the expression (1.27) [43]. In order to evaluate the integral of Eq. (1.42) we need to inset the condensate wave function, which in the simple 
case of isotropic potential $\left(\sigma_{z}=\sigma_{\rho}\right)$ becomes a product of three Gaussian distributions with equal widths $\sigma$. Therefore the Fourier transform of the condensate density is readily calculated as

$$
\widetilde{\rho}(\mathbf{k})=\frac{N}{\left(\sqrt{\pi} \sigma a_{\mathrm{ho}}\right)^{3}} \int \mathrm{d}^{3} r e^{-i \mathbf{k} \cdot \mathbf{r}} e^{-\frac{\mathbf{r}^{2}}{\sigma^{2} a_{\mathrm{ho}}^{2}}}=\exp \left[-\frac{\sigma^{2} a_{\mathrm{ho}}^{2}}{4} \mathbf{k}^{2}\right] .
$$

This expression has to be inserted into the integral (1.42), which can be easily evaluated in polar $(r, \gamma, \varphi)$ coordinates $^{2}$, giving

$$
\begin{aligned}
E_{\mathrm{dd}} & =\frac{N C_{\mathrm{dd}}}{2} \int \sin \gamma \mathrm{d} \gamma \mathrm{d} \varphi k^{2} \mathrm{~d} k\left(\cos ^{2} \gamma-1 / 3\right) \exp \left[-\frac{\sigma^{2} a_{\mathrm{ho}}^{2}}{2} \mathbf{k}^{2}\right] \\
& =N C_{\mathrm{dd}} 2 \pi \int \mathrm{d} k k^{2} \exp \left[-\frac{\sigma^{2} a_{\mathrm{ho}}^{2}}{2} \mathbf{k}^{2}\right] \int_{-1}^{+1} \mathrm{~d} x\left(x^{2}-1 / 3\right) \\
& =0,
\end{aligned}
$$

where we have performed the change of variable $x=\cos \gamma$. The generalization to anisotropic density distributions is mathematically more demanding but in principle straightforward, and leads to Eq. (1.36).

\subsubsection{Extended Bose-Hubbard model}

As in section 1.2.2, we expand the field operators in the basis of Wannier functions (1.19), and we keep only the lowest index corresponding to the first Bloch band. Within this approximation the first line of Eq. (1.29) leads to the Bose-Hubbard Hamiltonian (1.24). Instead the dipolar term gives rise to a further contribution

$$
\hat{H}_{\mathrm{dd}}=\frac{1}{2} \iint \mathrm{d}^{3} r_{1} \mathrm{~d}^{3} r_{2} \hat{\psi}^{\dagger}\left(\mathbf{r}_{1}\right) \hat{\psi}^{\dagger}\left(\mathbf{r}_{2}\right) U_{\mathrm{dd}}\left(\mathbf{r}_{1}-\mathbf{r}_{2}\right) \hat{\psi}\left(\mathbf{r}_{1}\right) \hat{\psi}\left(\mathbf{r}_{2}\right) .
$$

Therefore we only need to add to the Bose-Hubbard Hamiltonian (1.24) the terms arising from the dipolar part (1.46), after the expansion of the field operators in the basis of Wannier functions. In this basis the last expression becomes

$$
\hat{H}_{\mathrm{dd}}=\sum_{i, j, k, l} \frac{V_{i, j, k, l}}{2} \hat{a}_{i}^{\dagger} \hat{a}_{j}^{\dagger} \hat{a}_{k} \hat{a}_{l}
$$

where the matrix elements $V_{i, j, k, l}$ are given by the integral

$V_{i, j, k, l}=\iint \mathrm{d}^{3} r_{1} \mathrm{~d}^{3} r_{2} w^{*}\left(\mathbf{r}_{1}-\mathbf{R}_{i}\right) w^{*}\left(\mathbf{r}_{2}-\mathbf{R}_{j}\right) U_{\mathrm{dd}}\left(\mathbf{r}_{1}-\mathbf{r}_{2}\right) w\left(\mathbf{r}_{1}-\mathbf{R}_{k}\right) w\left(\mathbf{r}_{2}-\mathbf{R}_{l}\right)$.

\footnotetext{
${ }^{2}$ remember, $\gamma$ is the angle between the polarization axis $z$ and $\mathbf{k}$.
} 
The Wannier functions are centered at the bottom of the optical lattice wells with a spatial localization that we assume to be $\sigma$. For deep enough optical potentials we can assume $\sigma$ to be much smaller than the optical lattice spacing $d$, i.e. $\sigma \ll d$. In this limit, each function $w\left(\mathbf{r}-\mathbf{R}_{i}\right)$ is significantly non-zero for $\mathbf{r} \sim \mathbf{R}_{i}$, and the integral (1.48) is significantly non-zero for the indices $i=k$ and $j=l$. Therefore there are two main contributions to the integral (1.48): the off-site matrix element $V_{i, j, i, j}$ corresponding to $k=i \neq j=l$, and the on-site $V_{i, i, i, i}$ when all the indices are equal. Below we will explain the physical meaning of these two contributions.

Off-site - The dipolar potential $U_{\mathrm{dd}}\left(\mathbf{r}_{1}-\mathbf{r}_{2}\right)$ changes slowly on the scale of $\sigma$, therefore one may approximate it with the constant $U_{\mathrm{dd}}\left(\mathbf{R}_{i}-\mathbf{R}_{j}\right)$ and take it out of the integration. Then the integral reduces to

$$
V_{i, j, i, j} \simeq U_{\mathrm{dd}}\left(\mathbf{R}_{i}-\mathbf{R}_{j}\right) \int \mathrm{d}^{3} r_{1}\left|w\left(\mathbf{r}_{1}-\mathbf{R}_{i}\right)\right|^{2} \int \mathrm{d}^{3} r_{2}\left|w\left(\mathbf{r}_{2}-\mathbf{R}_{j}\right)\right|^{2},
$$

which leads to the off-site Hamiltonian

$$
\hat{H}_{\mathrm{dd}}^{\mathrm{off}-\text { site }}=\sum_{i \neq j} \frac{V_{i, j}}{2} \hat{n}_{i} \hat{n}_{j} .
$$

In the last expression $V_{i, j}=U_{\mathrm{dd}}\left(\mathbf{R}_{i}-\mathbf{R}_{j}\right), \hat{n}_{i}=\hat{a}_{i}^{\dagger} \hat{a}_{i}$ is the bosonic number operator at site $i$, and the sum runs over all different sites of the lattice.

On-site - At the same lattice site $i$, where $\left|\mathbf{r}_{1}-\mathbf{r}_{2}\right| \sim \sigma$, the dipolar potential changes very rapidly and diverges for $\left|\mathbf{r}_{1}-\mathbf{r}_{2}\right| \rightarrow 0$. Therefore the above approximation is not valid any more and the integral

$$
V_{i, i, i, i}=\iint \mathrm{d}^{3} r_{1} \mathrm{~d}^{3} r_{2} \rho\left(\mathbf{r}_{1}\right) U_{\mathrm{dd}}\left(\mathbf{r}_{1}-\mathbf{r}_{2}\right) \rho\left(\mathbf{r}_{2}\right)
$$

with $\rho(\mathbf{r})=|w(\mathbf{r})|^{2}$ being the single particle density, has to be calculated taking into account the atomic spatial distribution at the lattice site, similarly to what has been described in Sec. 1.3.2 ${ }^{3}$. We have already encountered this kind of integral in Sec. 1.3.2, and we have seen that, a part from a factor of 2, the solution can be found by Fourier transforming, i.e.

$$
V_{i, i, i, i}=\frac{1}{(2 \pi)^{3}} \int \mathrm{d}^{3} k \widetilde{U_{\mathrm{dd}}}(\mathbf{k}) \widetilde{\rho}^{2}(\mathbf{k}) .
$$

\footnotetext{
${ }^{3}$ Since $\mathbf{R}_{i}$ is a constant, we have renamed the variables as $\mathbf{r}_{u}-\mathbf{R}_{i}=\mathbf{r}_{u}$ for $u=1,2$.
} 
Which leads to an on-site dipolar contribution to the Hamiltonian of the type

$$
\hat{H}_{\mathrm{dd}}^{\mathrm{on}-\mathrm{site}}=\sum_{i} \frac{V_{i, i, i, i}}{2} \hat{n}_{i}\left(\hat{n}_{i}-1\right) .
$$

The extended Bose-Hubbard Hamiltonian is given by the sum of the BoseHubbard (1.24) and the dipolar Hamiltonians calculated above, leading to the expression

$$
\hat{H}_{\mathrm{eBH}}=-J \sum_{\langle i j\rangle} \hat{a}_{i}^{\dagger} \hat{a}_{j}+\frac{U}{2} \sum_{i} \hat{n}_{i}\left(\hat{n}_{i}-1\right)-\sum_{i} \mu_{i} \hat{n}_{i}+\sum_{i \neq j} \frac{V_{i, j}}{2} \hat{n}_{i} \hat{n}_{j},
$$

where $U$ is now taken into account as an effective on-site interaction

$$
U=g \int \mathrm{d}^{3} r|w(\mathbf{r})|^{4}+\frac{1}{(2 \pi)^{3}} \int \mathrm{d}^{3} k \widetilde{U_{\mathrm{dd}}}(\mathbf{k}) \widetilde{\rho}^{2}(\mathbf{k})
$$

which contains the contribution of the contact potential, with $g$ given in Eq. (1.12), plus the dipolar contribution coming from (1.53). Approximating each lattice site with a tiny harmonic trap, and approximating the atomic density distribution with Gaussians, $U$ looks like Eq. (1.38), and one can see that the resulting on-site interaction can be increased or decreased by changing the lattice confinement. 


\section{Chapter 2}

\section{Hubbard models: theoretical methods}

\subsection{Superfluid-Mott insulator quantum phase transition in the Bose-Hubbard model}

Consider the Bose-Hubbard Hamiltonian as derived in Sec. (1.2.2),

$$
\hat{H}_{\mathrm{BH}}=-J \sum_{\langle i j\rangle} \hat{a}_{i}^{\dagger} \hat{a}_{j}+\frac{U}{2} \sum_{i} \hat{n}_{i}\left(\hat{n}_{i}-1\right)-\mu \sum_{i} \hat{n}_{i},
$$

with a uniform chemical potential $\mu$, and a total number of Bosons given by the expectation value of the operator $\hat{N}=\sum_{i} \hat{n}_{i}$. There are three parameters in this Hamiltonian, namely $J, U$ and $\mu$, but it is a convention to reduce the analysis of the phase diagram of $\hat{H}_{\mathrm{BH}}$ to the ratio of two of them over the third one, e.g. $J / U$ and $\mu / U$.

The ground state of Hamiltonian (2.1) is easily understood for two opposite regimes of parameters: (i) for shallow lattices, i.e. $U / J \ll 1$, the system is in a gapless superfluid phase $(S F)$ characterized by on-site density fluctuations and the particles delocalized over the whole lattice; (ii) for deep lattices, i.e $J / U \ll 1$, and commensurate filling, on-site density fluctuations are completely suppressed, each site is occupied by an integer number of atoms $\bar{n}$, and the ground state is a product of single-site Fock states

$$
|G S\rangle=|\bar{n}, \bar{n}, \cdots\rangle
$$

This filling is energetically favorable in the range of chemical potential $\bar{n}-1 \leq$ $\mu / U \leq \bar{n}$. The system is gapped and incompressible, as beautifully explained in the famous paper of Fisher et. al. [1], and it is called a Mott insulator 
$M I(\bar{n})$. For small values of $J / U$ the $M I(\bar{n})$ phase persists in a closed and finite area of the $J$ vs. $\mu$ plane [1], which is called the Mott lobe for $M I(\bar{n})$. The larger $J / U$ value of the lobe is called the tip of the lobe or also critical point $(J / U)_{\mathrm{c}}$. The critical point changes with the dimensionality and geometry of the system.In Fig. 2.1 we plot the first $\bar{n}=0,1,2,3$ insulating lobes, calculated for an infinite optical lattice within the mean-field approximation, which will be discussed in Sec. 2.2.2. The thick black lines enclose the lobes and mark the boundaries between the $M I$ and $S F$ phases. Outside the insulating lobes, the phase is $S F$. The colored lines of Fig. 2.1(a) indicate a contour plot of constant fractional density, while the thick black lines departing from the tip of the lobes and extending into the $S F$ region, correspond to an integer value $\bar{n}$ of the density.

(a)

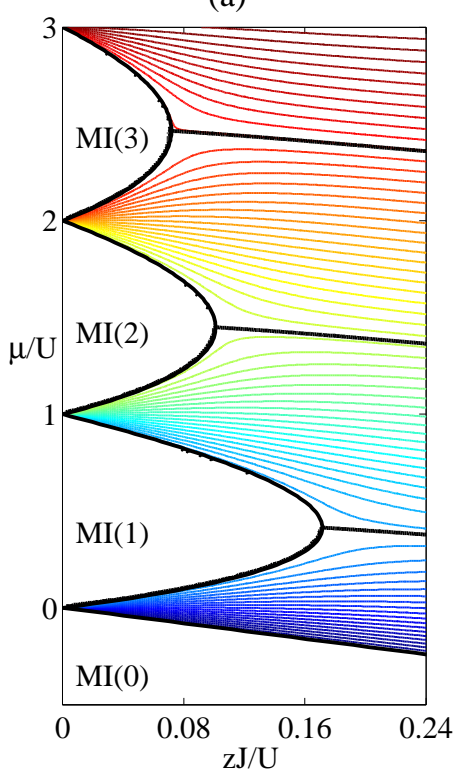

(b)

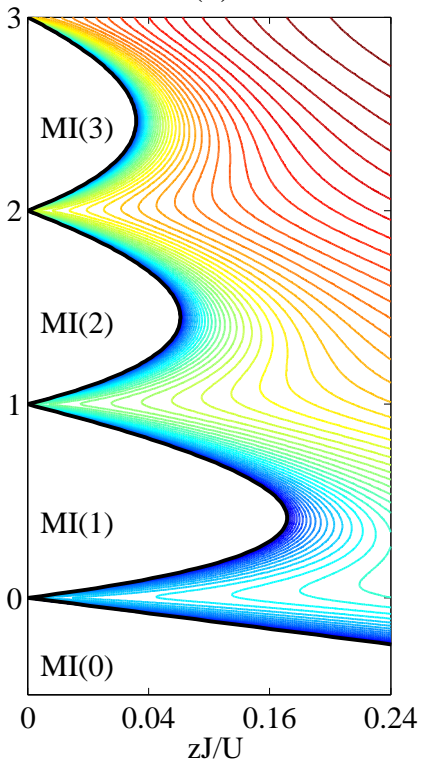

Figure 2.1: Mean-field phase diagram of the Bose Hubbard Hamiltonian. (a) Contour plot of the density per site; (b) contour plot of the order parameter (see Sec. 2.2). $M I(\bar{n})$ indicate a Mott insulating phase with fixed $\bar{n}$ atoms per site.

We will derive the mean-field Mott insulating lobes of Fig. 2.1 in a more rigorous way in Sec. 2.2.2, but for the moment we just list the critical points $(J / U)_{\mathrm{c}}$, for the $\bar{n}=1$ lobe, that have been estimated with different methods and for different dimensions of the lattice. In one dimension, the critical point has been estimated to be $(J / U)_{\mathrm{c}} \simeq 0.29$ [48] using Density Ma- 
trix Renormalization Group calculations (DMRG). In two dimensions, with quantum Monte Carlo calculations, the critical point has been estimated to be $(J / U)_{\mathrm{c}} \simeq 0.061[49]$, while in the three dimensional model the location of the critical point has been estimated with perturbative expansions to be at $(J / U)_{\mathrm{c}} \simeq 0.034[50]$. In the next section we will derive the mean-field lobes.

\subsection{The Gutzwiller mean-field approach}

The Gutzwiller mean-field approach is an approximation of the many-body wave function of Hubbard-type Hamiltonians and is given by

$$
|\Psi\rangle=\prod_{i} \sum_{n=0}^{n_{\max }} f_{n}^{(i)}|n\rangle_{i},
$$

where $|n\rangle_{i}$ represents the Fock state of $n$ atoms occupying the site $i, n_{\max }$ is a cut off in the maximum number of atoms per site, and $f_{n}^{(i)}$ is the probability amplitude of having the site $i$ occupied by $n$ atoms. The probability amplitudes are normalized to unity $\sum_{n}\left|f_{n}^{(i)}\right|^{2}=1$. The wave function (2.3) has been extensively used in the literature [22, 51, 52], and is motivated by its physical predictions; in fact, there exists a critical value $(J / U)_{\mathrm{mf}}$ in a given range of $\mu$, below which the ground state predicted by the Gutzwiller Ansatz is a product of single Fock states $f_{n}^{(i)}=\delta_{n, \bar{n}}$ with exactly $\bar{n}$ particles per site, as (2.2). Moreover, for $J / U>(J / U)_{\mathrm{mf}}$ the Gutwiller Ansatz predicts a superfluid ground state with fluctuating on-site particle number. The Gutzwiller critical point, for $\bar{n}=1$, is found to be $(J / U)_{\mathrm{mf}}=1 / 5.8 z$ [53], where $z=\sum_{\langle j\rangle_{i}} 1$ is the number of nearest neighbor connections at each site of the lattice. In table (2.2) we show the comparison of the critical points predicted by the Gutzwiller Ansatz for different dimensions of the system, with the more precise ones discussed in Sec. (2.1). From the comparison,

\begin{tabular}{cccc}
$\mathrm{D}$ & $z$ & $(J / U)_{\mathrm{mf}}$ & $(J / U)_{\mathrm{c}}$ \\
\hline \hline 1 & 2 & 0.0862 & 0.29 \\
2 & 4 & 0.0431 & 0.061 \\
3 & 6 & 0.0287 & 0.034 \\
\hline \hline
\end{tabular}

Table 2.1: Comparison of the Gutzwiller critical points $(J / U)_{\mathrm{mf}}$ with the more precise, up to now, critical points $(J / U)_{\mathrm{c}}$, for different dimensions D of the system.

one can deduce that the Guzwiller is unsatisfactory for 1D systems $(z=2)$ 
while it is satisfactory for a $3 \mathrm{D}$ one $(z=6)$. Also, in the limit of $J / U \rightarrow \infty$ the difference between the Gutzwiller predictions and the exact results are negligible [53]. Summarizing, the Gutzwiller predictions are exact in the two limiting cases of $J / U \rightarrow 0$ and $J / U \rightarrow \infty$, while for intermediate cases the performance of the Gutzwiller approach strongly depends on the dimension of the lattice, since it does not correctly account for the quantum fluctuations at the phase transition.

An important quantity is the so called order parameter, which is the expectation value of the Bosonic annihilation operator at the $i$-th site of the lattice, namely $\varphi_{i}=\left\langle\Psi\left|\hat{a}_{i}\right| \Psi\right\rangle$, and by using the Gutzwiller wavefunction (2.3) one gets

$$
\varphi_{i}=\sum_{n} \sqrt{n+1} f_{n}^{*(i)} f_{n+1}^{(i)} .
$$

The order parameter $\varphi_{i}$ describes the phase of the system at the site $i$ of the lattice: it is exactly zero in the Mott phase $\varphi_{i}=0$, while it assumes a non zero value $\varphi_{i} \neq 0$ in the superfluid phase. In the uniform system, the lattice is translationally invariant and therefore all sites are self-similar, which means that a single order parameter determines the phase of the whole system. In Fig. 2.1(b) we plot the absolute value of the order parameter $\varphi_{i}$ for such a system, in the $J / U$ vs. $\mu / U$ plane. The colored lines outside the insulating lobes correspond to a contour plot of constant non-zero value of $\varphi_{i}$ typical of the $S F$ phase. Instead, in a non-uniform system as it is in the presence of an external confining harmonic potential, different phases can coexist. As an example, in Fig. 2.2 we plot the density of the ground state (a) along with the order parameter at each site (b), of a 2D lattice in the presence of a confining harmonic potential. Notice that the $M I$ phase at the center of the harmonic trap $\left(x_{0}, y_{0}\right)$ is surrounded by a ring of $S F$ phase, in a wedding-cake like structure, as first discussed in [22]. The ground state of Fig. 2.2 was obtained within the mean-field approximation through the imaginary-time evolution technique, that will be discussed in Sec. 2.2.1.

In the presence of dipolar interactions, as we shall see later on, it is also necessary to account for non-uniform quantum phases, because even in the uniform system the presence of dipolar interactions may lead to spontaneous symmetry breaking of translational invariance on a scale larger than the lattice constant.

\subsubsection{Dynamical Gutzwiller approach}

The time dependent version of the Gutzwiller wavefunction (2.3) is obtained by allowing the Gutzwiller amplitudes to depend on time $f_{n}^{(i)}(t)$ [54]. Then 

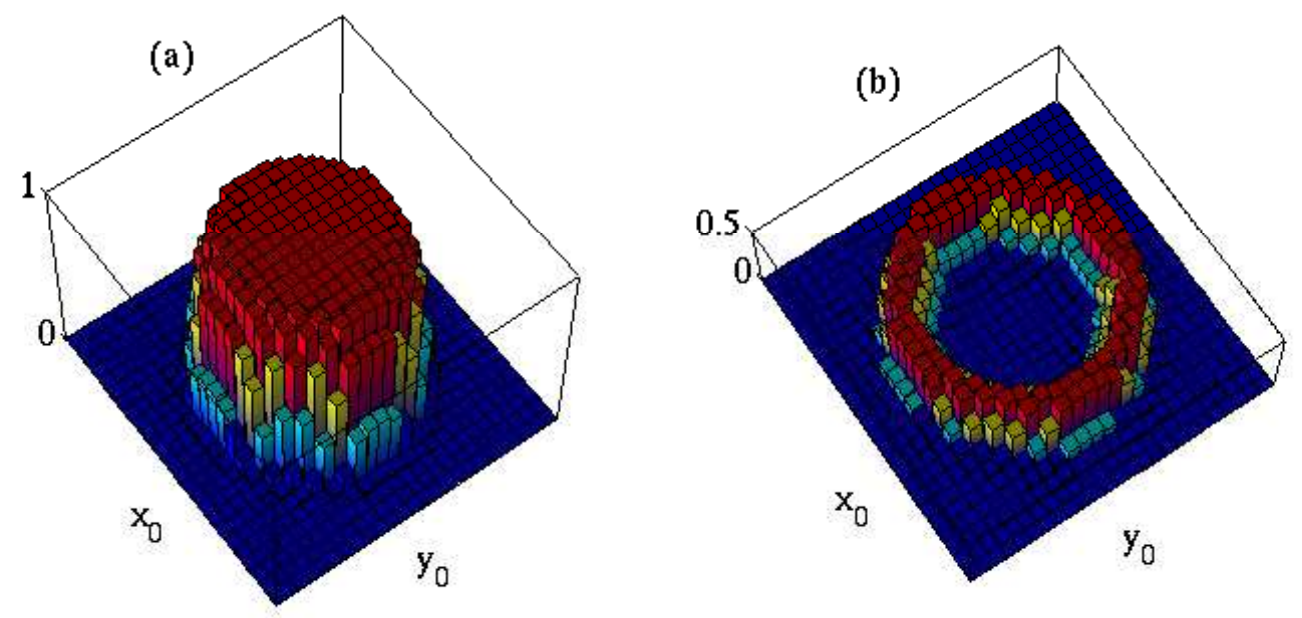

Figure 2.2: Mean-field ground state of a 2D optical lattice. (a) the vertical axis shows the value of the density at each site of the lattice, and the corresponding order parameter is in (b). The value of the harmonic oscillator frequency is given by $\Omega=0.0108 \times 2 \pi U / \hbar$.

the equations of motion for the amplitudes are readily obtained by minimizing the action of the system, given by $S=\int \mathrm{d} t \mathcal{L}$, with respect to the variational parameters $f_{n}^{(i)}(t)$ and their complex conjugates $f_{n}^{*(i)}(t)$. The Lagrangian of the system in the quantum state $|\Psi\rangle$, is given by [60]

$$
\mathcal{L}=\frac{\langle\Psi \mid \dot{\Psi}\rangle-\langle\dot{\Psi} \mid \Psi\rangle}{2 i}-\langle\Psi|\hat{H}| \Psi\rangle
$$

where $|\dot{\Psi}\rangle$ is the time derivative of the wave function (2.3). By equating to zero the variation of the action with respect to $f_{n}^{*(i)}$, one gets the equations

$$
\begin{aligned}
i \hbar \frac{\mathrm{d}}{\mathrm{d} t} f_{n}^{(i)}= & -J\left[\bar{\varphi}_{i} \sqrt{n} f_{n-1}^{(i)}+\bar{\varphi}_{i}^{*} \sqrt{n+1} f_{n+1}^{(i)}\right] \\
& +\left[\frac{U}{2} n(n-1)+n \sum_{j \neq i} V_{i, j}\left\langle\hat{n}_{j}\right\rangle-\mu_{i} n\right] f_{n}^{(i)},
\end{aligned}
$$

where $\bar{\varphi}_{i}=\sum_{\langle j\rangle_{i}} \varphi_{j}$, the sum runs over all nearest neighbors $j$ of site $i$, $\left\langle\hat{n}_{j}\right\rangle=\left\langle\Psi\left|\hat{a}_{j}^{\dagger} \hat{a}_{j}\right| \Psi\right\rangle$ is the average particle number at site $j$, and the total number of particles is given by $N=\sum_{i}\left\langle\hat{n}_{i}\right\rangle$. It is not difficult to verify the commutation relation $\left[\hat{N}, \hat{H}_{\mathrm{BH}}\right]=0$, which implies that the total number of 
Bosons is a conserved quantity for dynamics in the real time [46]. These equations are of mean-field type, because they are written for a single site $i$ and the "field" $\bar{\varphi}_{i}$ together with $\sum_{j \neq i} V_{i, j}\left\langle\hat{n}_{j}\right\rangle$, represent the influence of neighboring sites on the site $i$, and have to be determined self-consistently. Eqs. (2.6) are also a set of coupled equations, the coupling arising from the tunneling part, and can be written in the matrix form

$$
i \hbar \frac{\mathrm{d}}{\mathrm{d} t} \vec{f}=\mathcal{M}[\vec{f}, \mu, U, J] \cdot \vec{f}
$$

where $\vec{f}=\left[f_{0}^{(1)}, f_{1}^{(1)}, \cdots, f_{n}^{(i)}, \cdots f_{n_{\max }}^{\left(N_{S}\right)}\right]^{\mathrm{T}}$, is the vector of the Gutzwiller amplitudes ordered from site 1 to site $N_{S}$, the latter being the total number of sites. It is worth noticing that the matrix $\mathcal{M}[\vec{f}, \mu, U, J]$ is itself a functional of the coefficients $\vec{f}$ through the fields $\bar{\varphi}_{i}$ and $\sum_{j \neq i} V_{i, j}\left\langle\hat{n}_{j}\right\rangle$, which have to be calculated in a self-consistent way. Let us clarify this point with an example. Suppose we want to solve Eq. (2.7) between an initial time $t_{i}=0$ and a final time $t_{f}$, with a given initial condition $\vec{f}(0)$. We discretize the time interval in $N$ steps of size $\Delta t$, with $N$ finite, and define $t_{s}=s \Delta t$ such that $t_{s=0} \equiv 0$ and $t_{s=N} \equiv t_{f}$. Therefore, to calculate the solution at a certain point in time $\vec{f}\left(t_{s+1}\right)$ we need to know the solution right at the preceding time $\vec{f}\left(t_{s}\right)$, with which we can compute the fields that in turn determine $\mathcal{M}\left[\vec{f}\left(t_{s}\right), \mu, U, J\right]$, and the solution is readily found to be

$$
\vec{f}\left(t_{s+1}\right)=e^{-i \mathcal{M}\left[\vec{f}\left(t_{s}\right), \mu, U, J\right] \Delta t / \hbar} \vec{f}\left(t_{s}\right) .
$$

Starting from $s=0$, in $N+1$ steps we have determined the solution at the desired time $t_{f}$. At the computational level, this is the simplest procedure one can implement to calculate the dynamics of the system. However, one needs to be careful in the choice of the time step $\Delta t$, especially for fastoscillating dynamics. In such cases, a Runge-Kutta with adaptive stepsize control has proven to be more efficient. Instead for stiff dynamics, where the solution presents both slowly-varying and fast oscillating regions, the simple procedure described above may be enough accurate as in the case of imaginary time evolution.

Equations (2.7) can be solved in real time $t$ or also imaginary time $\tau=$ it. The imaginary time evolution is a standard technique that has been thoroughly used, because due to dissipation is supposed to converge to the ground state of the system. Two things are worth to be noticed. First, because the imaginary time evolution is not unitary, it does not conserve the norm of the Gutzwiller wavefunction, which has to be renormalized after each time step. Second, the total number of particles is not a conserved quantity 
any more. For dipolar Hamiltonians the imaginary time evolution does not always converge to the true ground state and it gets blocked in configurations which are a local minimum of the energy. On the one hand this makes it a difficult task to identify the ground state of such systems, and on the other hand it is a signature of the existence of metastable states as we will discuss in details in the next part.

\subsubsection{Perturbative mean-field approach}

A more convenient method to determine the insulating phases of a dipolar Hamiltonian is to use a mean-field approach perturbative in $\varphi_{i}$. From statistical mechanics, the expectation value of the annihilation operator at the $i$-th site is given [56] by the trace

$$
\varphi_{i}=\left\langle\hat{a}_{i}\right\rangle=\operatorname{Tr}\left(\hat{a}_{i} \hat{\rho}\right)
$$

where $\hat{\rho}=Z^{-1} e^{-\beta \hat{H}}$ is the density matrix operator, $Z=\operatorname{Tr}\left(e^{-\beta \hat{H}}\right)$ its normalization, and $\beta=1 / K_{B} T$ is the inverse temperature of the system. We write Hamiltonian (1.54) in the form $\hat{H}=\hat{H}_{0}+\hat{H}_{1}$ where

$$
\begin{aligned}
\hat{H}_{0} & =\frac{U}{2} \sum_{i} \hat{n}_{i}\left(\hat{n}_{i}-1\right)-\mu \sum_{i} \hat{n}_{i}+\sum_{i \neq j} \frac{V_{i, j}}{2} \hat{n}_{i} \hat{n}_{j} \\
\hat{H}_{1} & =-J \sum_{\langle i j\rangle} \hat{a}_{i}^{\dagger} \hat{a}_{j},
\end{aligned}
$$

and we assume a uniform chemical potential $\mu$. The generalization to a sitedependent chemical potential is straightforward. Furthermore, we assume low temperatures $\beta \rightarrow \infty$, and the tunneling coefficient to be the smallest energy in the system, i.e $J \ll U, \mu, V_{i, j}$ such that we can treat $\hat{H}_{1}$ as a small perturbation on $\hat{H}_{0}$, and use the Dyson expansion at the first order in $\hat{H}_{1}$ for all the exponential operators, so that one obtains

$$
e^{-\beta\left(\hat{H}_{0}+\hat{H}_{1}\right)} \simeq e^{-\beta \hat{H}_{0}}\left[\hat{\mathbb{1}}-\int_{0}^{\beta} e^{\tau \hat{H}_{0}} \hat{H}_{1} e^{-\tau \hat{H}_{0}} \mathrm{~d} \tau\right]
$$

We now write Hamiltonian (2.11) as a sum of single site Hamiltonians. Writing the annihilation operator as $\hat{a}_{i}=\hat{A}_{i}+\varphi_{i}$, we can perform the mean field decoupling on the tunneling term

$$
\begin{aligned}
\hat{a}_{i}^{\dagger} \hat{a}_{j} & =\hat{A}_{i}^{\dagger} \varphi_{j}+\hat{A}_{j} \varphi_{i}+\varphi_{i} \varphi_{j}+\hat{A}_{i}^{\dagger} \hat{A}_{j} \\
& \simeq \hat{a}_{i}^{\dagger} \varphi_{j}+\hat{a}_{j} \varphi_{i}-\varphi_{i} \varphi_{j},
\end{aligned}
$$


where in the last step we have assumed small fluctuations, characteristic of the Mott, or the deep superfluid states, and replaced $\hat{A}_{i}^{\dagger} \hat{A}_{j} \simeq 0$. In Hamiltonian (2.11) we now replace $\hat{a}_{i}^{\dagger} \hat{a}_{j}$ with the expression calculated above, we neglect terms of the order of $\varphi^{2}$ and find the mean field tunneling Hamiltonian

$$
\hat{H}_{1}^{\mathrm{MF}}=-J \sum_{i}\left(\hat{a}_{i}^{\dagger} \bar{\varphi}_{i}+\bar{\varphi}_{i}^{*} \hat{a}_{i}\right) .
$$

Given a classical distribution of atoms in a lattice such as

$$
|\Phi\rangle=\prod_{i}\left|n_{i}\right\rangle_{i}
$$

satisfying $\hat{H}_{0}|\Phi\rangle=E_{\Phi}|\Phi\rangle$, let us suppose that this configuration is a local minimum of the energy, it can be the ground state, namely the absolute minimum, or another local minimum. We will be more rigorous at the end of this section regarding the meaning of local minimum of energy but for the moment let us refer to the common picture of a local minimum. In the basis of the eigenfunctions of $\hat{H}_{0}$, satisfying the relation $\hat{H}_{0}|\Upsilon\rangle=E_{\Upsilon}|\Upsilon\rangle$ the partition function then takes the simple form

$$
Z \simeq \operatorname{Tr}\left(e^{-\beta \hat{H}_{0}}\right)=\sum_{|\Upsilon\rangle}\left\langle\Upsilon\left|e^{-\beta \hat{H}_{0}}\right| \Upsilon\right\rangle \stackrel{\beta \mapsto \infty}{\longrightarrow} e^{-\beta E_{\Phi}}
$$

where the last limit holds because we do not trace over all the state of the basis but only around the state $|\Phi\rangle$, which is assumed to be a local minimum of the energy. Using again a Dyson expansion of the exponential of the density operator, we obtain the order parameter as

$$
\begin{aligned}
\varphi_{i} & \simeq-e^{\beta E_{\Phi}} \int_{0}^{\beta} \operatorname{Tr}\left[\hat{a}_{i} e^{-(\beta-\tau) \hat{H}_{0}} \hat{H}_{1}^{\mathrm{MF}} e^{-\tau \hat{H}_{0}}\right] \mathrm{d} \tau \\
& =J \bar{\varphi}_{i} e^{\beta E_{\Phi}} \int_{0}^{\beta} \sum_{|\Upsilon\rangle}\left\langle\Upsilon\left|\hat{a}_{i} e^{-(\beta-\tau) \hat{H}_{0}} \hat{a}_{i}^{\dagger} e^{-\tau \hat{H}_{0}}\right| \Upsilon\right\rangle
\end{aligned}
$$

which is easy to calculate. The trace is then non trivial only for $|\Upsilon\rangle=|\Phi\rangle$ and $|\Upsilon\rangle=\frac{\hat{a}_{i}}{\sqrt{n_{i}}}|\Phi\rangle$, where $n_{i}$ is integer on $|\Phi\rangle$, and after the integration in the $\beta \mapsto \infty$ limit we are left with the result

$$
\varphi_{i}=J \bar{\varphi}_{i}\left[\frac{n_{i}+1}{E_{\mathrm{P}}^{i}}+\frac{n_{i}}{E_{\mathrm{H}}^{i}}\right],
$$

where the quantities $E_{\mathrm{P}}^{i}, E_{\mathrm{H}}^{i}$ are defined as

$$
\begin{aligned}
& E_{\mathrm{P}}^{i}=-\mu+U n_{i}+V_{\mathrm{dip}}^{1, i} \\
& E_{\mathrm{H}}^{i}=\mu-U\left(n_{i}-1\right)-V_{\mathrm{dip}}^{1, i},
\end{aligned}
$$


and are respectively the energy cost for a particle $(\mathrm{P})$ and hole $(\mathrm{H})$ excitation on top of the $|\Phi\rangle$ configuration. In the previous expressions $V_{\text {dip }}^{1, i}=\sum_{j \neq i} V_{i, j} n_{j}$ is the dipole-dipole interaction that feels one atom placed at site $i$, with the rest of the atoms in the lattice. We performed the integral (2.17) in the limit of $\beta \mapsto \infty$, and in such a limit one finds that the integral converges only for positive values of the particle and hole excitation energies, namely

$$
U\left(n_{i}-1\right)+V_{\text {dip }}^{1, i}<\mu<U n_{i}+V_{\text {dip }}^{1, i}
$$

This requirements have to be fulfilled at every site $i$ of the lattice and they simply state that the configuration $|\Phi\rangle$ is a local minimum with respect to adding and removing particles at any site. In the light of this statement, the restriction on the trace of Eq. (2.16) is now rigorous, and is in perfect agreement with the treatment done in [1]. Notice, that if $|\Phi\rangle$ is not a local minimum, then one finds that conditions (2.20) are never satisfied and the integral (2.17) indeed diverges. This treatment is of course also valid for $|\Phi\rangle$, being in particular the ground state of the system.

One finds such an equation (2.18), and conditions (2.20) for every site $i$ of the lattice. The convergence conditions are simple and among them one has to choose the most stringent to find the boundary of the lobe at $\mathrm{J}=$ 0 . Instead the equations for the order parameters are coupled due to the $\bar{\varphi}_{i}$ term, they can be written in a matrix form $\mathcal{M}(\mu, U, J) \cdot \vec{\varphi}=0$, with $\vec{\varphi} \equiv\left(\cdots \varphi_{i} \cdots\right)$, and have a non trivial solution. For every $\mu$, the smallest $J$ for which $\operatorname{det}[\mathcal{M}(\mu, U, J)]=0$ gives the lobe of the $|\Phi\rangle$ configuration in the $J$ vs. $\mu$ plane.

\subsubsection{Perturbative mean-field vs. dynamical Gutzwiller approach}

The predictions of the perturbative mean-field treatment are in perfect agreement with the results of the dynamical Gutzwiller approach, since they both rely on the same mean-field approximations. The first looks at the stability of a given density distribution $|\Phi\rangle=\prod_{i}\left|n_{i}\right\rangle_{i}$ of integer $n_{i}$ atoms per site, with respect to particle and hole excitations, while the latter minimizes the energy of a random initial configuration with respect to particle and hole excitations leading to the distribution $|\Phi\rangle$ if the initial condition is sufficiently close. However, the first method can only identify the phase boundaries of the insulating lobes without providing any further information on the SF phases outside the lobes, which can instead be explored with the imaginary time evolution. Nevertheless for dipolar Hamiltonians, due to the presence of many local minima of the energy, as we will see in the next part $[57,58]$, 
it is very difficult to identify the ground state with the dynamical Gutzwiller approach. This can be achieved more efficiently through the perturbative mean-field approach. Therefore the two methods complement each other. As an example, in Fig $2.1(\mathrm{a}, \mathrm{b})$ the black lines are calculated with the perturbative method $\left(V_{i, j}=0\right)$ while the $\mathrm{SF}$ region outside the lobes is explored using imaginary time evolution showing perfect agreement with the two approaches. 


\section{Part II}

\section{Metastable states}




\section{Introduction}

In this part of the thesis we use the theoretical methods described previously to investigate the physics of polarized dipolar Bosons in a two-dimensional optical lattice. Due to the long-range character of dipole-dipole interaction, the phase diagram of this system presents exotic quantum phases, like checkerboard and supersolid phases. In this thesis, we have considered the properties of the system beyond its ground state, and found that it is characterized by a multitude of almost degenerate metastable states, similarly to a disordered system.

The work is organized as follows. In Sec. 3.1 we describe our model and the Hamiltonian of the system. In Sec. 3.2 we give our definition of metastability, calculate the mean-field ground state phase diagram of the system, and show that in our treatment, metastable states appear as soon as one introduces at least one nearest neighbor of the dipolar interaction. The stability and lifetime of these states due to tunneling, is studied in Sec. 3.3, using a generalization of the instanton theory, and a variational Ansatz. We discuss how these state can be prepared on demand, and how they can be detected in Sec. 3.4, while in Sec. 3.5 we discuss the effect of a harmonic confinement.

Our original work is based on the following publications:

- C. Menotti, C. Trefzger, and M. Lewenstein, Metastable States of a Gas of Dipolar Bosons in a 2D Optical Lattice. Physical Review Letters, 98, 235301 (2007).

- C. Trefzger, C. Menotti, and M. Lewenstein, Ultracold dipolar gas in an optical lattice: The fate of metastable states. Physical Review A, 78, 043604, (2008). 


\section{Chapter 3}

\section{Dipolar Bosons in a 2D optical lattice}

\subsection{The model}

In $[57,58]$, we have studied the properties of dipolar Bosons in an infinite 2D optical lattice, mimicked by an elementary cell of finite dimensions $L \times$ $L\left(N_{S}=L^{2}\right.$ sites $)$ satisfying periodic boundary conditions. The dipoles are aligned and point perpendicularly out of the plane so that the dipoledipole interaction (1.28) between two particles at relative distance $\mathbf{r}$ becomes $U_{\mathrm{dd}}(\mathbf{r})=C_{\mathrm{dd}} /\left(4 \pi r^{3}\right)$ repulsive and isotropic in the $2 \mathrm{D}$ plane of the lattice, where $C_{\mathrm{dd}}$ is given by Eq. (1.27). Furthermore for computational simplicity we truncate the range of the off-site interactions at a finite number of nearest neighbors, as shown in Fig. 3.1 up to the range number four.

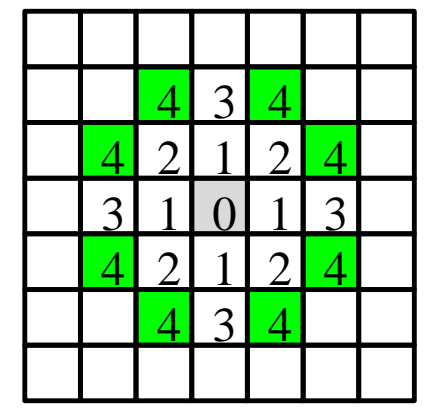

Figure 3.1: Representation of the first four nearest neighbors of the site labeled as 0 in the $2 \mathrm{D}$ lattice. 
We have studied the phase diagram of the system described by the Hamiltonian

$$
\hat{H}=-J \sum_{\langle i j\rangle} \hat{a}_{i}^{\dagger} \hat{a}_{j}-\sum_{i} \mu \hat{n}_{i}+\sum_{i} \frac{U}{2} \hat{n}_{i}\left(\hat{n}_{i}-1\right)+\frac{U_{\mathrm{NN}}}{2} \sum_{\vec{\ell}} \sum_{\langle\langle i j\rangle\rangle_{\vec{\ell}}} \frac{1}{|\vec{\ell}|^{3}} \hat{n}_{i} \hat{n}_{j},
$$

where $U_{\mathrm{NN}}=C_{\mathrm{dd}} /\left(4 \pi d_{2 \mathrm{D}}^{3}\right)$ is the dipole-dipole interaction between nearest neighboring sites, $d_{2 \mathrm{D}}$ is the lattice period, and $\langle\langle i j\rangle\rangle_{\vec{\ell}}$ represents neighbors at

relative distance $\vec{\ell}$ which is measured in units of $d_{2 \mathrm{D}}$. All the other quantities were introduced previously.

\subsection{Metastability}

Let us start the discussion by introducing our definition of stability of a given classical distribution of atoms in the lattice, i.e. a product over single-site Fock states

$$
|\Phi\rangle=\prod_{i}\left|n_{i}\right\rangle_{i}
$$

At $J=0$, we define the state (3.2) to be stable if there exists a finite interval $\Delta \mu=\mu_{\max }-\mu_{\min }>0$ in the $\mu$ domain, in which the particle $(\mathrm{P})$ and hole $(\mathrm{H})$ excitations at each site $i$ of the lattice are positive, and the system is gapped. Using the dipolar Hamiltonian (3.1), in Sec. 2.2.2 we have calculated the particle and hole excitation energies of $|\Phi\rangle$ to be

$$
\begin{aligned}
& E_{\mathrm{P}}^{i}=-\mu+U n_{i}+V_{\mathrm{dip}}^{1, i} \\
& E_{\mathrm{H}}^{i}=\mu-U\left(n_{i}-1\right)-V_{\mathrm{dip}}^{1, i},
\end{aligned}
$$

where we recall $V_{\text {dip }}^{1, i} \geq 0$ to be the dipolar interaction experienced by one atom sitting at the site $i$ of the lattice. From Eqs. (3.3) it is then straightforward to find $\Delta \mu$, if it exists, given by the set of inequalities

$$
U\left(n_{i}-1\right)+V_{\text {dip }}^{1, i}<\mu<U n_{i}+V_{\text {dip }}^{1, i}
$$

This is consistent with the stability conditions discussed in the seminal paper of Fisher et. al. [1]. Indeed, in the absence of dipolar interactions $U_{\mathrm{NN}}=0$ into Eqs. (3.4), one recovers the well known conditions $U\left(n_{i}-1\right)<\mu<U n_{i}$ for the stability of the $M I\left(n_{i}\right)$, with $n_{i}$ particles per site. One can extend the stability analysis also for small values of $J$, and for a given stable state calculate its insulating lobe with the perturbative mean-field approach we have developed in Sec. 2.2.2. In this context, we therefore define a state like 
(3.2) to be metastable if it satisfies two conditions: the first is that the state must have an insulating lobe inside which it is gapped, and the second is that the energy of the state must be higher than the ground state energy. In other words a metastable state is a local minimum of the energy.

In the absence of dipolar interactions $U_{\mathrm{NN}}=0$, no metastable states are found. In the low tunneling region the ground state of the system consists of Mott insulating lobes with integer filling factors $\nu=N_{\mathrm{a}} / N_{\mathrm{S}}$ (number of atoms/number of sites), while for large values of $J$ the system is superfluid. In our treatment metastable states appear as soon as one introduces at least one nearest neighbor of the dipolar interaction. In fact, the imaginary time evolution, which for Bose Hubbard Hamiltonians with only on-site interactions converges unambiguously to the ground state, for the dipolar Hamiltonian (3.1) often converges to different metastable configurations depending on the exact initial condition. Moreover, in the real time evolution, their stability manifests as typical small oscillations of frequency $\omega_{0}$ around the local minimum of the energy.

Our main results $[57,58]$ are summarized in Figs. 3.2 (a,b,c), where we plot the phase diagram of the Hamiltonian (3.1) for a $L=4$ elementary cell satisfying periodic boundary conditions, for different values of the cut off range in the dipolar interactions respectively at one (a), two (b), and four nearest neighbors. The on-site interaction is given by $U / U_{\mathrm{NN}}=20$ and $U_{\mathrm{NN}}=1$ is the unit of energy.

The thick lines correspond to the ground state while the thin lines correspond to metastable insulating states, with the color identifying the same filling factor $\nu$. The difference with the Bose Hubbard phase diagram of Fig. 2.1, where only integer filling factors $\nu=\bar{n}$ are present, is evident already with one nearest neighbor of the dipolar interaction (a). In fact the $M I(1)$ lobe undergoes a global shift of $z U_{\mathrm{NN}}(z=4$ in the figure) towards higher values of $\mu$, and the new fractional filling factor $\nu=1 / 2$ appears with a ground state density distribution modulated in a checkerboard pattern, shown in Fig. 3.2 (GS) with white empty sites and gray sites occupied by one atom. Instead, the density distribution shown in Fig. $3.2(\mathrm{I})$ is metastable with $\nu=1 / 2$, and its insulating lobe is given by the thin line extending from $1<\mu / U_{\mathrm{NN}}<3$. Remarkably, in Fig. 3.2(a) the two lobes extending from $0<\mu / U_{\mathrm{NN}}<1$ correspond to metastable configurations at filling factors $\nu=1 / 4,5 / 16$, while the two lobes between $3<\mu / U_{\mathrm{NN}}<4$ correspond to metastable states at filling factors $\nu=3 / 4,11 / 16$, but no ground state is found for these fillings. In the region immediately outside the ground state lobes, we found evidences of Supersolid $(S S)$ phases, where the order parameters $\varphi_{i}$ are different than zero and are spatially modulated, e.g. in a $C B$ structure. Before our work, studies 

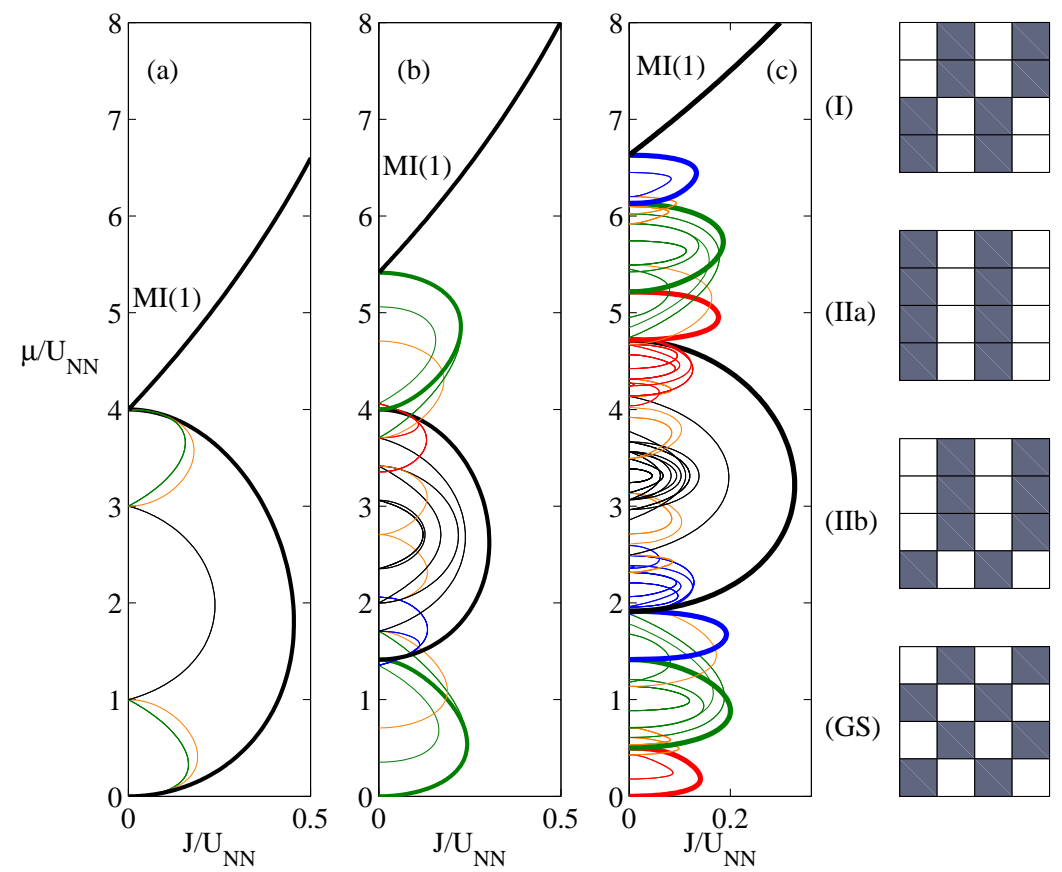

Figure 3.2: (a), (b), (c) Phase diagram with a range of the dipole-dipole interaction cut at the first, second, and fourth nearest neighbor, respectively. The thick line is the ground state and the other lobes correspond to the metastable states, the same color corresponding to the same filling factor. In (c) filling factors range from $\nu=1 / 8$ to $\nu=1$. In the right column we present metastable configurations for $\nu=1 / 2$ appearing at the first nearest neighbor (I), and second (IIa, IIb), and the corresponding ground state (GS); those metastable states remain stable for all larger ranges of the dipole-dipole interaction.

of $\mathrm{BH}$ models with extended interactions have pointed out the existence of novel quantum phases, like the $S S$ and checkerboard phases, but not the existence of the metastable states.

Increasing further the range of the dipolar interactions leads to the appearance of more metastable states, as (IIa) and (IIb) found at $\nu=1 / 2$ for two nearest neighbors in the range of dipolar interactions. The $M I(1)$ undergoes a larger shift which is accompanied by the emergence of other insulating fractional filling factors, as shown in Fig. 3.2 (c), where the dipole-dipole interaction is cut at the fourth nearest neighbor and the ground state is a series of lobes with $\nu$ multiple of $\nu=1 / 8$. The number of metastable states varies 
depending on the parameters of the Hamiltonian and the filling factor; it is found to be up to 400 for $U / U_{\mathrm{NN}}=20$ at filling $\nu=1 / 2$ as shown in Fig. 3.3 (a), and up to 1500 for $U / U_{\mathrm{NN}}=2$ at unit filling. With this picture in mind, it is now clear why the imaginary time evolution, which often converges to different metastable configurations, is very inefficient both to find the ground state of the system and to compute the lobe boundaries of a given metastable state. Instead, the mean-field perturbative approach we have derived in Sec. 2.2.2 has proven to be satisfactory for this purpose but it also has some limitations. In fact, all possible values of $\nu$ and the corresponding configurations which are detectable with this method is limited by the size of the elementary cell. Evidently the possible filling factors of an elementary cell of size $L$ are given by multiples of $\nu=1 / L^{2}$. It is worth to notice, that despite the inefficiency of the imaginary time evolution in finding the insulating lobes of the system, the corresponding equations in real time turn out to be very useful, for example, to compute the excitation spectrum of the system as explained in the appendix A.

As shown in Fig. 3.3 (b), we found that there is usually a gap between the ground state and the lowest metastable state, which might allow to reach the ground state by ramping up the optical lattice under some adiabaticity condition. However, this feature is strongly reduced in the case of larger elementary cells because the number of metastable configurations and the variety of their patterns increase very rapidly with the size of the elementary cell $L$. Indeed, we have found that there exist many metastable configurations that differ from the ground state only by small localized defects, and the energy of these reduces the size of the gap.

\subsection{The lifetime}

We have studied the stability of the metastable states with a path integral formulation in imaginary time and a generalization of the instanton theory [59]. For any given initial metastable configuration $|\Phi\rangle_{\text {initial, }}$, we are able to estimate the time $T$ in which $|\Phi\rangle_{\text {initial }}$ has tunneled completely into a different metastable state $|\Phi\rangle_{\text {final }}$, in analogy with the case of a classical particle tunneling through a potential barrier shown in Fig. 3.4 (a), with the difference that we do not have any information a priory on the characteristics of the potential barrier separating initial and final state. Nevertheless we can estimate the barrier and the time $T$ in three steps: (i) first we construct the imaginary time Lagrangian of the system described by a quantum state $|\Phi\rangle$, (ii) we make use of a variational method on $|\Phi\rangle$ with only one variational parameter $q$, and its conjugate momenta $P$, that interpolate continuously 

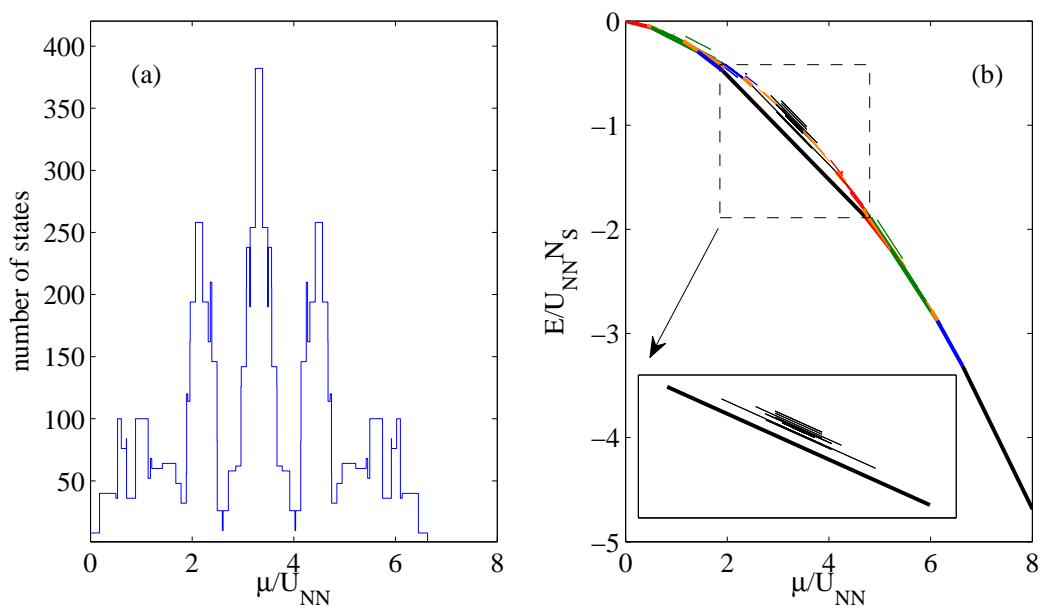

Figure 3.3: (a) Number of metastable states, and (b) energy of the ground (thick line) and metastable states (thin lines) as function of $\mu$, for for $U / U_{\mathrm{NN}}=20$, and a range of the dipole-dipole interaction cut at the fourth nearest neighbor. The inset shows the energy levels at filling factor $1 / 2$.

between $|\Phi\rangle_{\text {initial }}$ and $|\Phi\rangle_{\text {final }}$, and (iii) through the variation of $q$ we calculate the minimal action $S_{0}$, with the imaginary time Lagrangian, along the stationary path starting at $|\Phi\rangle_{\text {initial }}$; this path is called an instanton path, in short instanton. It connects $|\Phi\rangle_{\text {initial }}$ and $|\Phi\rangle_{\text {final }}$ only if the two states are degenerate, otherwise the stationary path connects $|\Phi\rangle_{\text {initial }}$ with an interme-

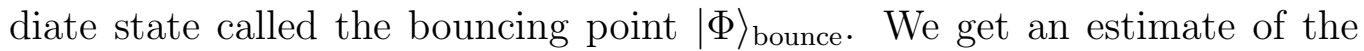
energy barrier separating the two states by evaluating the Lagrangian from $|\Phi\rangle_{\text {initial }}$ to $|\Phi\rangle_{\text {final }}$ and imposing zero "momentum" $P=0$, i.e. as one would do in the Lagrangian of a classical particle in a potential.

Once the minimal action $S_{0}$ is known, then the tunneling time $T$ is readily calculated [59] as

$$
\omega_{0} T=\frac{\pi}{2} e^{S_{0}}
$$

where $\omega_{0}$ is of the order of the frequency of the typical small oscillations of $|\Phi\rangle_{\text {initial }}$ around the local minimum of the energy. In analogy to a classical particle tunneling through a barrier, the instanton has the nice interpretation of the stationary path connecting the two local minima in the inverted potential, as schematically represented in Fig. 3.4 (b). 


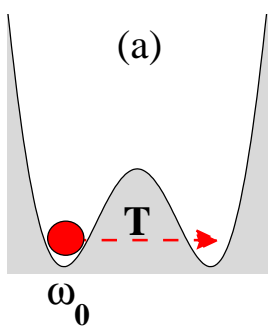

(b)

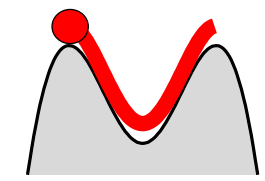

(c)

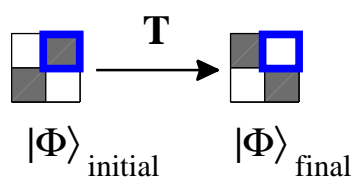

Figure 3.4: (a) Particle in a minimum of a potential barrier, the particle oscillates with frequency $\omega_{0}$ around the local minimum and tunnels into the right well in a time $T$; (b) the instanton; and (c) the process for which a checkerboard state tunnels into the anti-checkerboard that shown complete exchange of particle with holes and vice versa. The process happens in a time $T$ in analogy with (a).

The imaginary time Lagrangian of a system [60], described by a quantum state $|\Phi\rangle$, is given by

$$
\mathcal{L}=-\frac{\langle\dot{\Phi} \mid \Phi\rangle-\langle\Phi \mid \dot{\Phi}\rangle}{2}+\langle\Phi|\hat{H}| \Phi\rangle
$$

with $|\dot{\Phi}\rangle$ indicating the time-derivative, and $\hat{H}$ being the Hamiltonian of the system. In the approximation where $|\Phi\rangle$ is the Gutzwiller wave function of a given metastable state, we write its amplitudes as

$$
f_{\mathrm{n}}^{(i)}=\frac{1}{\sqrt{2}}\left(x_{\mathrm{n}}^{(i)}+i p_{\mathrm{n}}^{(i)}\right),
$$

where $x_{\mathrm{n}}^{(i)}$, and $p_{\mathrm{n}}^{(i)}$ are real numbers which are going to be related to the variational parameters, and their conjugate momenta in the following. For 
simplicity, we consider states with a maximum occupation number of $n_{\max }=$ 1 (i.e. $n=0,1$ ), and therefore we have a total of $4 N_{S}$ parameters with $N_{S}$ being the total number of sites. The Lagrangian (3.6) becomes a functional of the $4 N_{S}$ parameters, namely

$$
\mathcal{L}\left[x_{\mathrm{n}}^{(i)}, p_{\mathrm{n}}^{(i)}\right]=-i \sum_{i, n=0}^{1} p_{\mathrm{n}}^{(i)} \dot{x}_{\mathrm{n}}^{(i)}+\langle\Phi|\hat{H}| \Phi\rangle,
$$

as well as the expectation value of the Hamiltonian $\langle\Phi|\hat{H}| \Phi\rangle$. In order to put the Lagrangian (3.8) in its canonical form we must introduce the new coordinates $q_{\mathrm{n}}^{(i)}=x_{\mathrm{n}}^{(i)}$ and their conjugate momenta $P_{\mathrm{n}}^{(i)}=\partial \mathcal{L} / \partial \dot{q}_{\mathrm{n}}^{(i)}=-i p_{\mathrm{n}}^{(i)}$, and we can write

$$
\mathcal{L}\left[q_{\mathrm{n}}^{(i)}, P_{\mathrm{n}}^{(i)}\right]=\sum_{i, n=0}^{1} P_{\mathrm{n}}^{(i)} \dot{q}_{\mathrm{n}}^{(i)}-\mathcal{H}\left[q_{\mathrm{n}}^{(i)}, P_{\mathrm{n}}^{(i)}\right]
$$

where $\mathcal{H}\left[q_{\mathrm{n}}^{(i)}, P_{\mathrm{n}}^{(i)}\right]=-\langle\Phi|\hat{H}| \Phi\rangle$ is a constant of the motion ${ }^{1}$. We now want to reduce the dynamic described by the Lagrangian (3.9) to a one dimensional problem, described only by one variable $q$ and its conjugate momentum $P$. Through the variation of $(q, P)$ we want to describe the interchange between the state $|\Phi\rangle_{\text {initial }}$ and $|\Phi\rangle_{\text {final }}$, as for example the one represented in Fig. 3.4(c). In Sec. 3.3.1, we show how to reduce the number of variational parameters to one, $q$, and its conjugate momentum $P$, by making use of a variational Ansatz as well as the normalization condition on the coefficients (3.7) and the conservation of the total number of particles. These conditions enter into the expression of the Lagrangian (3.9) through Lagrange multipliers $\lambda_{c}$. Consequently the equations of motion given by $\dot{q}=\partial \mathcal{H} / \partial P$, and $\dot{P}=-\partial \mathcal{H} / \partial q$ are governed by an Hamiltonian which also includes the constraints as follows

$$
\mathcal{H}=\mathcal{H}[q, P]+\sum_{c} \lambda_{c} \mathcal{C}_{c}
$$

where an explicit expression for the conditions $\mathcal{C}_{c}$ will be given in Sec. 3.3.1. The action is then readily calculated along the stationary path of Eq. (3.10) as follows

$$
S_{0}=\int \mathcal{L}[q, P] \mathrm{d} \tau=\int_{\text {path }} \mathcal{L}[q, P] \frac{\mathrm{d} q}{\dot{q}}
$$

with $\dot{q}=\partial \mathcal{H} / \partial P$ from Eq. (3.10).

\footnotetext{
${ }^{1}$ note that in the analogy of a classical particle in a potential $V(x)$, the conserved quantity in the imaginary time would be $\mathcal{H}=\frac{P^{2}}{2 m}-V(x)$, which describes the particle's motion in the inverted potential.
} 


\subsubsection{Parametrization}

Through the variation of $(q, P)$, we aim at describing the interchange between the state $|\Phi\rangle_{\text {initial }}$ and $|\Phi\rangle_{\text {final }}$, as for example the one schematically represented in Fig. 3.4(c). During this process there are sites initially occupied that empty, like the blue-framed site of Fig. 3.4(c), which we call the (B)-site, and vice versa, like the site on the left of $B$, which is initially empty and occupied at the end, and we name the (A)-site. When the initial and final state are non-degenerate, as for example the case sketched in Fig. $3.5(\mathrm{~d})$, there are also sites that do not change and remain either full $(\mathrm{F})$ or empty (E).

During this process, the Gutwiller amplitudes (3.7) have to be normalized at each site, and the total number of particles has to be conserved, namely

$$
\begin{aligned}
\left|f_{0}^{(i)}\right|^{2}+\left|f_{1}^{(i)}\right|^{2} & =1, \forall i \\
\sum_{i=1}^{N_{S}}\left|f_{1}^{(i)}\right|^{2} & =N,
\end{aligned}
$$

where $N_{S}$ is the total number of sites. We choose $(q, P) \equiv\left(q_{0}^{\mathrm{B}}, P_{0}^{\mathrm{B}}\right)$ to be the variational parameters of the blue-framed site, and the normalization condition (3.12) together with the conservation of the number of particles (3.13) between $\mathrm{A}$ and $\mathrm{B}$ give us three coupled equations

$$
\begin{array}{r}
q^{2}-P^{2}+\left(q_{1}^{\mathrm{B}}\right)^{2}-\left(P_{1}^{\mathrm{B}}\right)^{2}=2 \\
\left(q_{0}^{\mathrm{A}}\right)^{2}-\left(P_{0}^{\mathrm{A}}\right)^{2}+\left(q_{1}^{\mathrm{A}}\right)^{2}-\left(P_{1}^{\mathrm{A}}\right)^{2}=2 \\
\left(q_{1}^{\mathrm{B}}\right)^{2}-\left(P_{1}^{\mathrm{B}}\right)^{2}+\left(q_{1}^{\mathrm{A}}\right)^{2}-\left(P_{1}^{\mathrm{A}}\right)^{2}=2 .
\end{array}
$$

As explained in [58], we make use of the following Ansatz

$$
\begin{aligned}
q_{1}^{\mathrm{A}} & =q \\
P_{1}^{\mathrm{A}} & =P \\
P_{0}^{\mathrm{A}} & =P_{1}^{\mathrm{B}}=-P \\
q_{0}^{\mathrm{A}} & =q_{1}^{\mathrm{B}}
\end{aligned}
$$

with which it is clear that at the value of $(q, P)=(0,0)$ corresponds a situation in which site A is empty and site B is full, while for $(q, P)=(\sqrt{2}, 0)$ the contrary is true. For degenerate initial and final states as in the case of Fig. 3.4(c), the remaining sites they either behave like A or B, which implies 
another set of conditions summarized as follows

$$
\begin{gathered}
q_{0}^{(i)}=q_{0}^{\mathrm{A}(\mathrm{B})} \\
P_{0}^{(i)}=P_{0}^{\mathrm{A}(\mathrm{B})} \\
q_{1}^{(i)}=q_{1}^{\mathrm{A}(\mathrm{B})} \\
P_{1}^{(i)}=P_{1}^{\mathrm{A}(\mathrm{B})}
\end{gathered}
$$

depending on whether the site $i$ is initially empty (A) or occupied (B). Instead, when the initial and final state are non-degenerate, as is the case considered in Fig. 3.5(d), there are also sites that we assume not to change and remain either full $(\mathrm{F})$ or empty $(\mathrm{E})$, For these sites the constraints are respectively given by

$$
\begin{aligned}
q_{0}^{\mathrm{F}} & =0 \\
q_{1}^{\mathrm{F}} & =2 \\
P_{0}^{\mathrm{F}} & =P_{1}^{\mathrm{F}}=0,
\end{aligned}
$$

and

$$
\begin{aligned}
q_{0}^{\mathrm{E}} & =2 \\
q_{1}^{\mathrm{E}} & =0 \\
P_{0}^{\mathrm{E}} & =P_{1}^{\mathrm{E}}=0 .
\end{aligned}
$$

All these conditions (3.14)-(3.18), which we name $\mathcal{C}_{c}$, enter explicitly into the calculation of Hamiltonian (3.10).

\subsubsection{Action and tunneling time}

In Fig. $3.5(\mathrm{a}, \mathrm{b})$ we plot the minimal action divided by the total number of sites $N_{S}$ of the cell, as a function of the tunneling coefficient $J$, for two different processes. The first one $(\mathrm{a}, \mathrm{c})$, in which initial and final state are degenerate, shows the exchange of particles with holes in the whole lattice, and is sketched in the lower part of Fig. 3.5 (c) where we also plot the potential barrier between initial and final state calculated as $-\mathcal{H}(q, P=0)$. Instead, in the second one $(b, d)$ the final state is the ground state, i.e. deeper in energy with respect to the initial state, and only a few sites of the lattice exchange particles with holes during the process, as sketched in the upper part of Fig. 3.5 (d) along with the potential barrier. A side remark, the point where the thick line of the barrier encounters the dashed line is the bouncing point $|\Phi\rangle_{\text {bounce. }}$ 

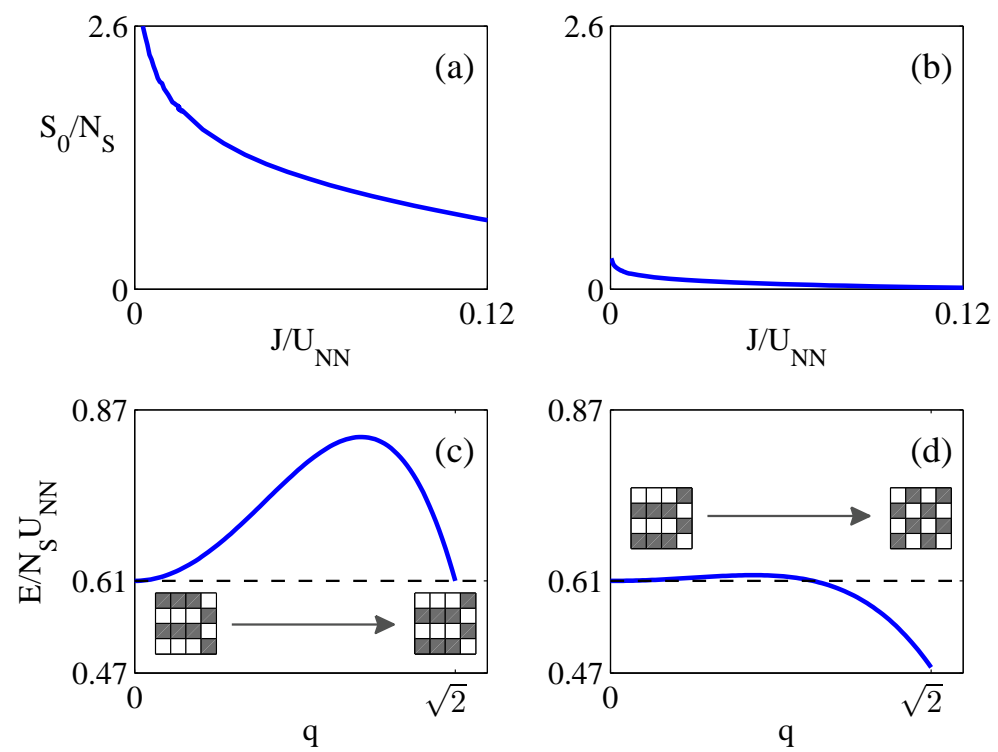

Figure 3.5: (a,b) Action per site and (c,d) energy barrier for the process sketched in panels $(\mathrm{c}, \mathrm{d})$. In both cases the initial state is the configuration (IIb) of Fig. 3.2 and the value $J=0.12 U_{\mathrm{NN}}$ corresponds to the tip of its insulating lobe. The first one $(\mathrm{a}, \mathrm{c})$ is for degenerate initial and final configurations while for the second one $(b, d)$ the final configuration is energetically deeper. The difference in the two processes manifests also in the height of the barrier which is smaller for the second case, leading to a smaller action and consequently a smaller life-time.

The action in general diverges for $J \rightarrow 0$ indicating a divergent tunneling time $T$, and then decreases monotonically up to a minimum value in correspondence of the tip of the lobe, $J=0.12 U_{\mathrm{NN}}$ here, signaling a minimum life-time at the tip of the lobe, as expected. In between these two extreme behaviors, the action increases monotonically with the number of sites involved in the exchange of particles with holes; the more sites involved as in the case of Fig. 3.5 (a,c), the bigger the action is. Summarizing, from the figures above, we conclude that small energy differences between the initial $|\Phi\rangle_{\text {initial }}$ and the final states $|\Phi\rangle_{\text {final }}$ and large regions of the lattice undergoing particle-hole exchange in the tunneling process contribute to large barriers, i.e. long life times $T$. On the contrary, for big energy differences and small regions of the lattice undergoing particle-hole exchange, the barrier is small. Hence, in general it is more likely for a given state to tunnel into a state deeper in energy, e.g., the ground state, than into its complementary, which 
implies the exchange of particles with holes in the whole lattice.

\subsection{Preparation, manipulation and detection}

Very important issues are the preparation and detection of the atomic states in the lattice. In the experiments with cold gases and optical lattices, the typical procedure is first to obtain a condensate in a harmonic trap and then adiabatically rump up the optical potential. Therefore one may ask the question how to reach a desired configuration or whether it is possible to reach the ground state in this way, since we have discussed in Sec. 3.2 that for large lattices there exist many configurations with localized defects that compete with the ground state.

One can use superlattices in order to prepare the atoms in configurations of preferential symmetry. We have checked that the presence of defects is strongly reduced when a local potential energy following desired patterns is added to the optical lattice. Note that the configurations obtained in such a way will also remain stable once the superlattice is removed, thanks to dipole-dipole interaction. Moreover, in [58] we have demonstrated that by using superlattices the transfer from one metastable configuration to another necessarily occurs via superfluid states, and can be controlled fully at the quantum level. As discussed in Sec. 3.4.1, the transfer is a quantum controlled process where the control parameters are among others the tunneling coefficient $J$, and the magnitude of the local chemical potential $\Delta \mu$ following a desired pattern. Even if at the $M I-S F$ transition it is impossible to be adiabatic because of the continuous excitation spectrum of the $S F$ phase [10], for a certain range of control parameters the process works, and as discussed in Sec. 3.4.1, is quite robust. In Fig. 3.6 we show an example of such a process in which we transfer the checkerboard ground state $(\mathrm{CB})$ into the metastable state (IIa) of Fig. 3.2, with a $99 \%$ fidelity.

The local chemical potentials follow the stripe pattern of the occupied sites of the metastable state (IIa), and are changed smoothly in time as shown in Fig. 3.6 (a). At the same time, the tunneling coefficient is varied as the smoothed step-like function of Fig. 3.6 (b) such as to exit the tip of the CB insulating lobe drawn as a dashed red line, remain in the $S F$ phase for an appropriate amount of time, and subsequently enter from the tip of the Mott insulating metastable state (IIa) represented by the blue thick line. Notice that since we are performing real-time dynamics, the total number of particles is a conserved quantity and therefore the MI to SF transition happens only at the tip of the insulating lobes. In Fig 3.6 (c) we plot the 

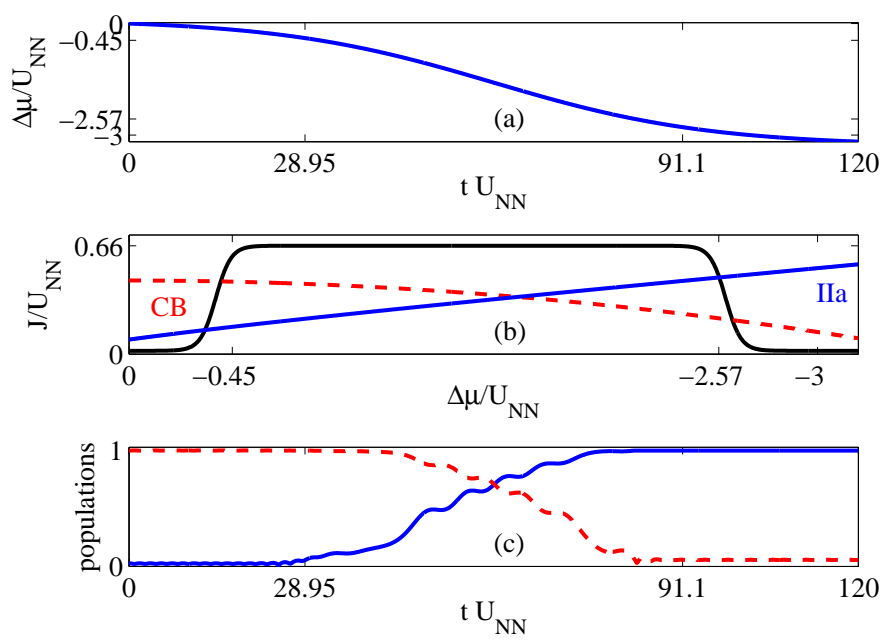

Figure 3.6: (a) The pulse of local chemical potential as a function of time. (b) The smoothed steplike function is the tunneling coefficient as a function of $\Delta \mu$, while the thick (dashed) line is the tip of the IIa (CB) insulating lobe. (c) Population inversion, from CB to IIa at the end of the process. Notice the oscillation of populations when passing through the $S F$ region of the phase diagram.

populations corresponding to the CB and (IIa) states defined as the $N_{S^{-}}$th root of the fidelity

$$
P_{\mathrm{MS}}=\sqrt[N_{S}]{\left|\left\langle\Phi_{\mathrm{MS}} \mid \Phi\right\rangle\right|^{2}}
$$

where $N_{S}$ is the number of sites of the elementary cell. The dashed line is the population of the $C B$ state while the thick line corresponds to the population of (IIa), which at the end of the process stabilizes at the value of $P_{\text {(IIa) }}=0.99$.

The spatially modulated structures created, and manipulated in such a way can be detected via the measurement of the noise correlations of the expansion pictures $[61,77,63]$ : the ordered structures in the lattice give rise to different patterns in the spatial noise correlation function

$$
\begin{aligned}
C(\vec{d}) & =\frac{\int \mathrm{d}^{2} x\left\langle\rho_{\mathrm{tof}}(\vec{x}+\vec{d} / 2) \rho_{\mathrm{tof}}(\vec{x}-\vec{d} / 2)\right\rangle}{\int \mathrm{d}^{2} x\left\langle\rho_{\mathrm{tof}}(\vec{x}+\vec{d} / 2)\right\rangle\left\langle\rho_{\mathrm{tof}}(\vec{x}-\vec{d} / 2)\right\rangle} \\
& \approx \sum_{j, k} \exp \left[i \frac{m}{\hbar t} \vec{d} \cdot\left(\vec{x}_{j}-\vec{x}_{k}\right)\right] \rho_{j} \rho_{k}=|\mathcal{F}(\rho)|^{2},
\end{aligned}
$$

where we have named $\rho_{\text {tof }}$ the density distribution after time of flight, while 
$\rho_{k}$ is the density distribution in the lattice. This is nothing else than the modulus square of the Fourier transform of the density distribution in the lattice. Such a measurement is in principle able to recognize the geometry of the density pattern in the lattice as well as the presence of defects in the density distribution, which could be exactly reconstructed starting from the patterns in the spatial noise correlation function. In Fig. 3.7 (I,II,II), the lower panels show the noise correlation functions for the density distributions at filling close to $1 / 2$ shown in the upper panels where we have assumed a localised Gaussian density distribution at each lattice site. The presence of defects in the density distribution can be in principle detected with this method.

For the moment, the signal to noise ratio required for single defect recognition is beyond present experimental possibilities. However, by averaging over a finite number of different experimental runs producing the same spatial distribution of atoms in the lattice, a good signal can be obtained.

Very recently it has been reported that, by means of a high-resolution optical imaging system described in [64], single atoms are detectable with near-unity fidelity on individual sites of a Hubbard-type optical lattice. The authors report a way to determine the presence of an atom on a single site of the lattice, by measuring the total number of scattered photons per lattice site. However, during the imaging process only empty or singly occupied sites can be seen in the image, because of molecule formation on multiply occupied sites and light-assisted collisions. This method could in principle be used to observe experimentally the different density distributions of the metastable states.

\subsubsection{Transfer process}

In [58] we have studied how to transfer population from a given metastable configuration to another one with a different symmetry, by changing the lattice parameters in time, where the dynamics have been described through the mean-field equations derived in Sec. 2.2.1. Specifically, we have studied how to transfer population from the CB state to the metastable state (IIa) of Fig. 3.2, by applying time dependent local chemical potentials in favor of the state (IIa), and by changing the tunneling coefficient $J$ in time, so as to exit the $\mathrm{CB}$ lobe and, through the superfluid region, enter into the (IIa) lobe. Ideally, the population of (IIa) at the end of the process has to be one, $P_{\text {(IIa) }}\left(t_{\text {fin }}\right)=1$, but the actual value of $P_{(\text {IIa })}\left(t_{f i n}\right)$ is very much sensitive on the exact values the parameters take during the dynamics. Specifically, the magnitude of the local chemical potentials is smoothly varied in time as 


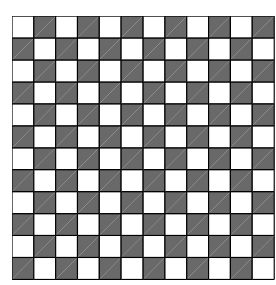

(I)

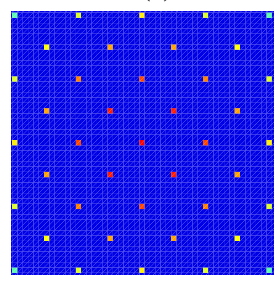

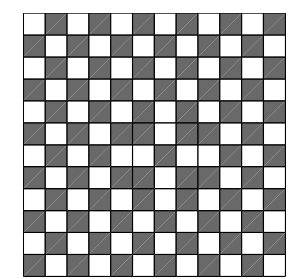

(II)

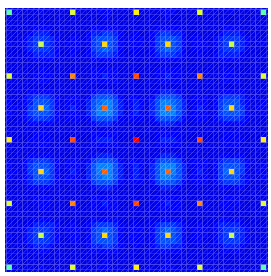

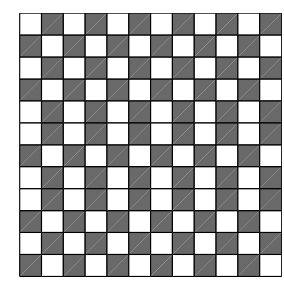

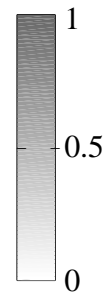

(III)

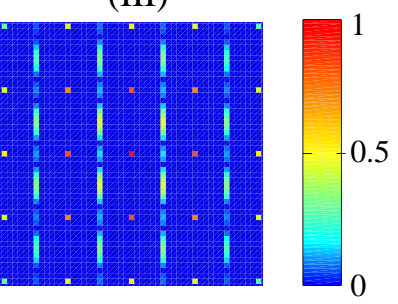

Figure 3.7: The lower panels show the spatial noise correlation patterns for configurations (I) to (III) in the upper pannels, assuming a localised Gaussian density distribution at each lattice site. Figure from [57].

follows

$$
\Delta \mu(t)=-C \tanh \left[\frac{\alpha}{C}\left(t-t_{0}\right)\right]+C \tanh \left[-\frac{\alpha}{C} t_{0}\right],
$$

where $C=1.7 U_{N N}$ and $t_{0}=60 / U_{N N}$ (in units of $\hbar=1$ ), are kept constant, while $\alpha$ is a free parameter that sets the maximum slope for this function. Instead, the tunneling coefficient is dynamically chanced as follows

$$
J(\Delta \mu)=\frac{J_{m}-J_{0}}{2} \min \left\{\begin{array}{l}
\tanh \left[-s m_{o}\right]-\tanh \left[s\left(\Delta \mu-m_{o}\right)\right]+\frac{2 J_{0}}{J_{m}-J_{0}} \\
\tanh \left[s\left(\Delta \mu-m_{i}\right)\right]-\tanh \left[-s m_{i}\right]+\frac{2 J_{m}}{J_{m}-J_{0}}
\end{array}\right.
$$

with $s=15 / U_{N N}$ and $J_{0}=0.02 U_{N N}$ constants, $m_{i}=-2.6 U_{N N}$ fixes the superfluid to Mott insulator transition point at $\Delta \mu_{i n}=-2.57 U_{N N}$, whereas $J_{m}$ and $m_{o}$ are free parameters related with the maximum value of tunneling coefficient in the superfluid region and the point $\Delta \mu_{o}$ where the CB ceases to exist. Together with the intensity $I_{r}$ of the random noise that fixes the initial condition, which, as discussed in [58], it is necessary in order not to have trivial dynamics, the space of our control parameters is in total 4dimensional, and is given by

$$
\left\{\alpha, \Delta \mu_{o}, J_{m}, I_{r}\right\}
$$


The control parameters for the dynamics shown in Fig. 3.6 are respectively given by $\alpha=40 \times 10^{-3} U_{N N}^{2}, \Delta \mu_{o}=-0.45 U_{N N}, J_{m}=0.66 U_{N N}$, and $I_{r}=4 \times$ $10^{-3}$, but having such a precise control on these parameters is experimentally very challenging. Nevertheless, such a process is robust if there is a reasonable range in which the parameters can vary without affecting the final result. The goal is of course the population of (IIa) to be as close as possible to 1 at the end of the process. We have discretized the space of parameters (3.23) arbitrarily, and for every value of the parameters we have simulated the dynamics represented in Fig. 3.6. The resulting statistics is shown in Fig. 3.8.
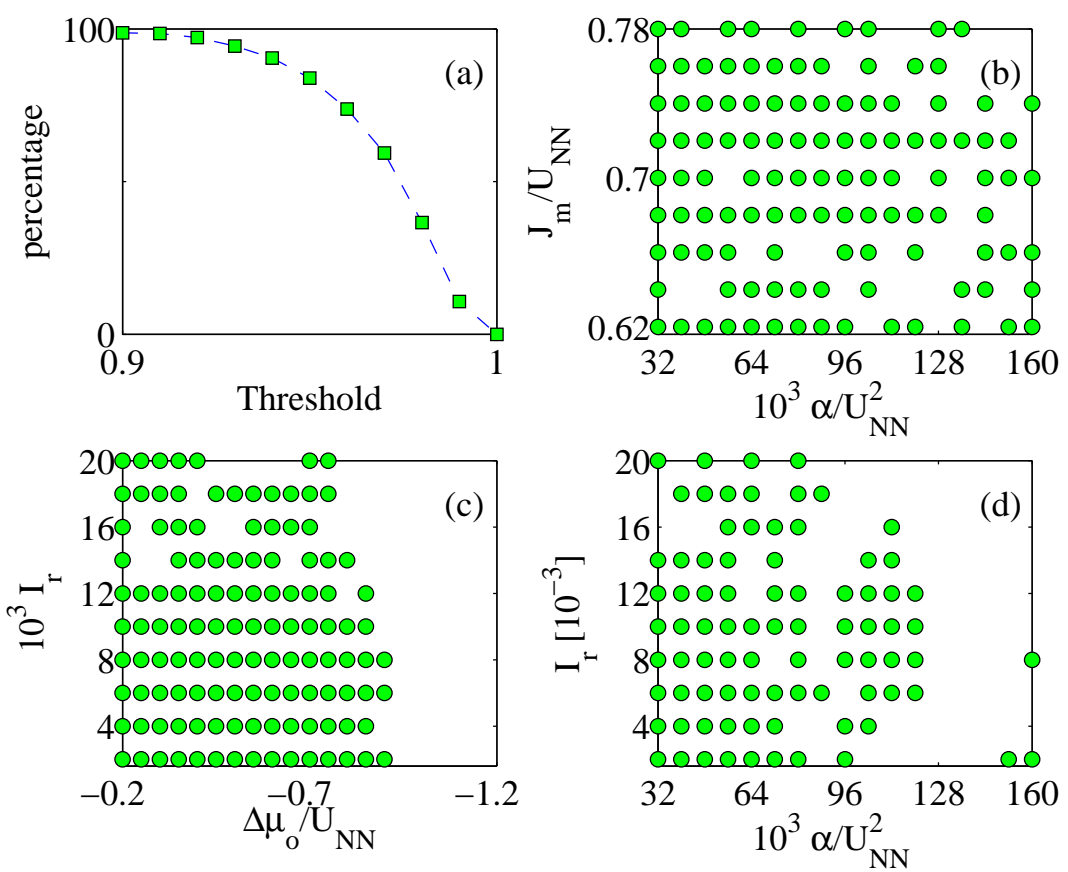

Figure 3.8: (a) Threshold versus the percentage of realizations terminating with a population of (IIa) bigger than threshold; as the threshold increases less realizations satisfy the required precision. (b-d) Slices of the discretized space of control parameters; the spots are for processes ending in (IIa) with at least 0.98 population. In (b) we fix $I_{r}=10 \times 10^{-3}$ and $\Delta \mu_{o}=-0.45 U_{N N}$, in (c) and (d) we fix $\alpha=40 \times 10^{-3} U_{N N}^{2}$ and $\Delta \mu_{o}=-0.45 U_{N N}$ respectively, for $J_{m}=0.66 U_{N N}$.

We have observed that there is a lower limit at $J_{m}=0.6 U_{N N}$, below which the transferring process does not work. For values of $J$ bigger than this limit, 
almost all the realizations end up in the (IIa) state with a final population bigger than 0.8 , but the exact value depends on the control parameters of the single realization. In Fig. 3.8 (a) we fix $J_{m}=0.66 U_{N N}$, and for any given value of the threshold on the horizontal axis, we plot the percentage of the dynamics which end up with a final population of the (IIa) state larger than the given threshold, i.e. $P_{(\mathrm{IIa})}\left(t_{f i n}\right) \geq$ threshold. As we increase the threshold the number of simulations ending up in (IIa) with a population that overcomes the given threshold decreases, up to no simulations ending up at the ideal value $P_{\text {(IIa) }}\left(t_{\text {fin }}\right)=1$. This is a clear signature of a quantum controlled process. Notice however, that about $36 \%$ of our simulations terminate with (IIa) being populated at 0.98 .

In Fig. $3.8(\mathrm{~b}, \mathrm{c}, \mathrm{d})$ we show slices of the hypercube defined by the discretized space of control parameters (3.23), where the spots are placed in correspondence of the values giving a dynamic with $P_{(\mathrm{IIa})}\left(t_{f i n}\right) \geq 0.98$. There is a closed region in the discretized $\left\{\alpha, \Delta \mu_{o}, J_{m}, I_{r}\right\}$ space in which one always comes through the 0.98 population threshold. This means that experimentally one has the freedom of setting the control parameters such that their small fluctuations do not affect the transfer process, and makes the specific process of population transferring from $\mathrm{CB}$ to (IIa) quite robust.

\subsection{Harmonic confinement}

In real experiments atoms first are trapped in a harmonic trap and then the optical lattice is raised. Therefore it is important to understand the behavior of these systems in the presence of a confining potential. Here we calculate the ground state of a finite $20 \times 20$ square lattice, where we superimpose a trapping potential mimicked by local chemical potentials [55], without periodic boundary conditions. The range of the dipole-dipole interaction is cut at the fourth nearest neighbor.

The external potential for the system in Fig. 3.9 is given by

$$
V(x, y)=\frac{K}{U_{\mathrm{NN}}}\left[\left(x-x_{0}\right)^{2}+\left(y-y_{0}\right)^{2}\right] \text {, }
$$

where $\left(x_{0}, y_{0}\right)$ is the center of the two-dimensional isotropic trap. On the two outermost sites of the lattice the potential is such to force the density to zero. In Fig. 3.9 (a) there is clearly a region around the center of the trap where the density $\rho(x, y)$ follows a checkerboard pattern, and where the superfluid

parameter $|\varphi(x, y)|^{2}$ is zero, as shown in Fig. 3.9 (b). Notice the supersolidsuperfluid area that surrounds the Mott insulating phase. Instead, in Figs. 

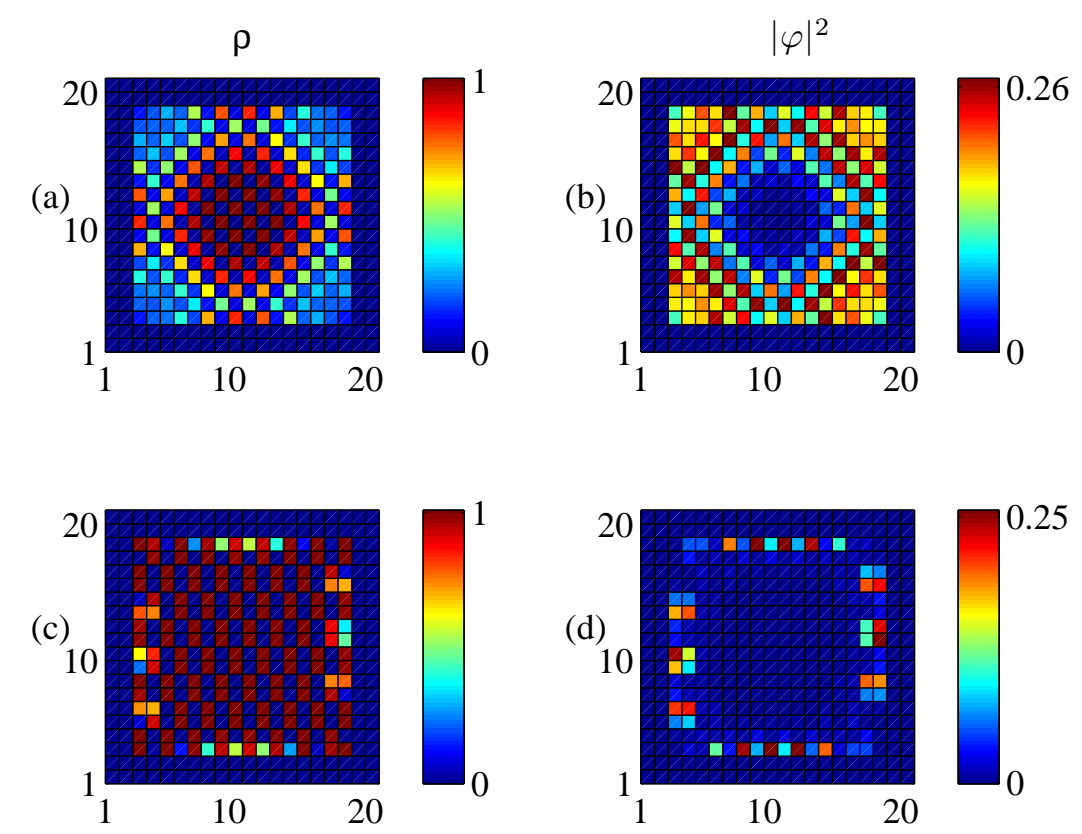

Figure 3.9: Density $\rho(x, y)$, and superfluid parameter $|\varphi(x, y)|^{2}$ in the harmonic potential of Eq. 3.24. The parameters of the system are given by $\mu / U_{\mathrm{NN}}=2.8, J / U_{\mathrm{NN}}=0.26$, and $K=107 \times 10^{-3} s^{-1}$ for panels (a),(b), while $\mu / U_{\mathrm{NN}}=3.3, J / U_{\mathrm{NN}}=0.16$, and $K=3.1 \times 10^{-3} s^{-1}$ for panels (c),(d) in units of $\hbar=1$.

3.9 (c), and (d), the density in the center of the trap follows the metastable state atomic distribution of Fig. 3.2 (I), with a zero superfluid parameter, while in the outer region of the trap a SF state is present. 


\section{Chapter 4}

\section{Conclusions}

In $[57,58]$ we have studied a single component gas of dipolar Bosons in a two-dimensional optical lattice. The atoms feature a polarized dipole moment perpendicular to the plane of the lattice resulting in a long range interaction repulsive in every direction of the plane. The dipole-dipole interaction range has been truncated at the fourth nearest neighbor, and we have considered a $4 \times 4$ unitary cell with periodic boundary conditions. We have shown that such a system possesses many almost degenerate metastable states competing with the ground state, and that the dipole-dipole interaction is the responsible for the appearance of these metastable states.

We have studied the stability of these states and have shown that the tunneling time scales exponentially with the action, with a factor which depends in a complicated way on the hopping parameter $J$, the energy difference between the two metastable states, and the number of lattice sites involved during the tunneling process. We also showed how to identify the state in the lattice through noise correlation measurements or by means of the highresolution optical imaging system described in [64].

The mean field theory calculations have shown that, once the system is prepared in one of the metastable states, it is necessary to go into the superfluid region of the phase diagram in order to break the symmetry of the prepared state and transfer it to another one. Even though this is a quantum controlled process, very much sensitive to the exact values of the control parameters during the dynamics, we have shown that the process is quite robust. The capability of initializing, reading and manipulating these systems makes dipolar Bosons in optical lattice very promising for applications in quantum information as quantum memories. 


\section{Part III}

Multiple layers and mixtures 


\section{Introduction}

In the previous part we have studied a single component gas of dipolar Bosons in a two-dimensional optical lattice, where the atoms featured a polarized dipole moment perpendicular to the plane of the lattice resulting in a long range interaction repulsive in every direction within the plane. We have shown that due to the presence of the dipole-dipole interaction, the phase diagram of the simple Bose Hubbard Hamiltonian where only $M I$ and $S F$ phases are present [1] is strongly modified in the low-tunneling region, by the appearance of new insulating checkerboard phases featuring fractional filling factors and a spatial modulation of the density, and at larger tunneling by the appearance of the $S S$ phases presenting modulated patterns of the non-zero order parameter. The $S F$ phase is still present for high-tunneling regions. Moreover, we have shown that the system possess many insulating metastable states often competing with the ground state and we have estimated their life time due to tunneling to other metastable states.

The aim of this part is to understand how the previous picture changes in a multi-layer structure where, due to the long range character of the dipole-dipole interaction, the atoms feel a dipolar interaction coming from the three directions. Since we consider polarized dipoles, the interaction is then anisotropic and in particular it will be attractive along the direction of polarization and repulsive in the directions perpendicular to the polarization axis. In a previous work [67] on polar molecules trapped in a 3D stack of strongly confined pancake traps, it has been shown that for sufficiently strong dipole moments, polarized in the direction perpendicular to the planes of the traps, the system is stable against collapse because tunneling between different planes is suppressed. Due to the attractive interaction between molecules in different layers the system undergoes BEC of dipolar chains along the polarization axis. This problem was analyzed by neglecting the interchain dipolar interactions. In a recent work [68], fermionic polar molecules confined in a stack of three 1D wires with no inter-wire tunneling were also studied, and it was found that bosonic dimers or fermionic trimers can form. 
In our work, we have studied a system composed of a stack of two 2D optical lattice layers with the dipoles polarized perpendicularly to the $2 \mathrm{D}$ planes (see Sec. 5.1). Because the tunneling between different layers is suppressed the gas is stable against collapse, and by including the attractive part of the dipolar interaction in the perpendicular direction we find that particles belonging to the two different planes may bind together in a composite. The composites feature not only the pair $M I$ and pair $S F$ phases but also a novel pair-supersolid $(P S S)$ quantum phase. In the context of Bose mixtures in a one-dimensional optical lattice, a small region of PSS phase was found in the presence of intra-species on-site repulsion, and on-site attraction between the two species [69].

The work is organized as follows. In Sec. 5.1 we introduce and explain the details of the model, we study the ground state of the system by means of the perturbative mean field approach derived in Sec. 2.2.2 based on a Gutzwiller Ansatz. We will see that this method is not accurate enough to describe the true ground state of the system, and the reason lays behind the nature of the lowest lying excitations. In fact, in the limit of parameters we consider, we demonstrate that it is energetically favorable to dope the system with a pair of particles (pair of holes) instead of a single particle (single hole).

In Sec. 5.2, we show that the system admits a description in terms of a low-energy subspace of pairs, we derive the effective Hamiltonian $\hat{H}_{\text {eff }}$ for the subspace by means of perturbation theory up to second order in tunneling. We derive a new perturbative mean field approach for $\hat{H}_{\text {eff }}$, similarly as in Sec. 2.2.2, which only admits pair of particles or pair of holes excitations and we find the insulating ground state lobes of the system.

In Sec. 5.3, we generalize the dynamical Gutzwiller approach derived in Sec. 2.2.1, and derive the dynamics equations for the Gutzwiller amplitudes in the low-energy subspace. These equations permit us to investigate the phases of the system outside the insulating lobes and we find strong evidences for the existence of a supersolid phase of pairs. We discuss the limit of validity of our description in terms of an effective Hamiltonian, and we conclude in chapter 7 .

Our results are based on the publication:

- C. Trefzger, C. Menotti, and M. Lewenstein, Pair-Supersolid Phase in a Bilayer System of Dipolar Lattice Bosons. Physical Review Letters, 103, 035304, (2009).

- C. Trefzger, M. Alloing, C. Menotti, F. Dubin, and M. Lewenstein, Counterflow Supersolid of anti-polarized dipolar Bosons in a 2D optical lattice. In preparation. 


\section{Chapter 5}

\section{Dipolar Bosons in a bilayer optical lattice}

\subsection{The model}

In [74] we consider polarized dipolar particles in two decoupled 2D optical lattice layers (see Fig. 5.1), where the potential barrier between the two layers is large enough to prevent any inter-layer hopping. This is the simplest multilayer structure and can be obtained by using anisotropic optical lattices or superlattices, which can exponentially suppress tunneling in one direction.

The in-plane dipolar interaction is isotropic and repulsive. The interlayer interaction depends on the relative position between the two dipoles, but is dominated by the nearest-neighbor attractive interaction $W<0$ between two atoms at the same lattice site in different layers. We include only nearestneighbor $(N N)$ in-plane $\left(U_{\mathrm{NN}}\right)$ and out-of-plane $(W)$ dipolar interactions. Since tunneling is suppressed between the layers particles belonging to the different layers cannot mix and behave in practice like two different species 1. The problem is analogous to that of two bosonic species on a $2 \mathrm{D}$ optical lattice with an inter-species attraction $W<0$ at the same lattice site, and intra-species repulsion $U_{\mathrm{NN}}$. The relative strength between $U_{\mathrm{NN}}$ and $W$ can be tuned by changing the spacing $d_{\perp}$ between the two layers, relative to the $2 \mathrm{D}$ optical lattice spacing $d$. Because of the dependence of the dipole-dipole interaction like the inverse cubic power of the distance, the ratio $|W| / U_{N N}$ can be tuned over a wide range. While it can be negligible for $d_{\perp} \gg d$ making the system asymptotically similar to a single $2 \mathrm{D}$ lattice layer, it can

\footnotetext{
${ }^{1}$ Because of this analogy we will often refer to the two layers as the two species and vice versa.
} 


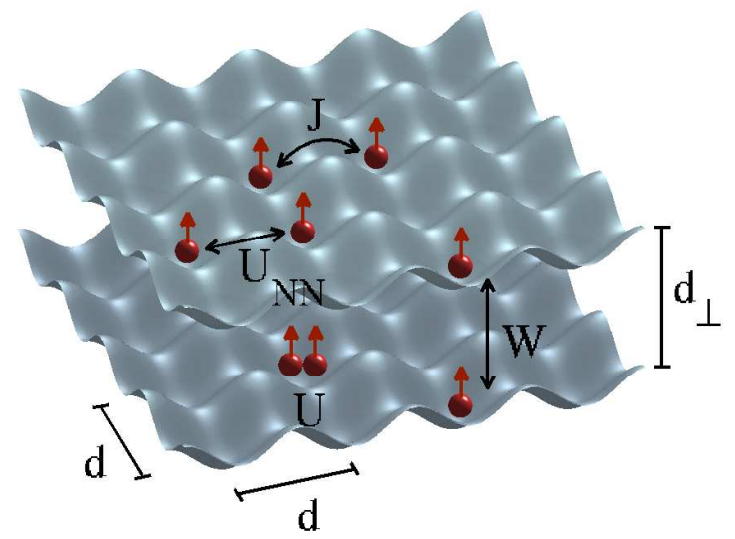

Figure 5.1: Schematic representation of two 2D optical lattice layers populated with dipolar bosons polarized perpendicularly to the lattice plane. The particles feel repulsive on site $U$ and nearest-neighbor $U_{\mathrm{NN}}$ interactions. Interlayer tunneling is completely suppressed, while a nearest-neighbor interlayer attractive interaction $W$ is present.

also become relevant and give rise to interesting physics, not existing in the single layer model as pointed out in $[65,66,67,70,71,72,73]$.

The system is described by the Hamiltonian

$$
\begin{aligned}
\hat{H} & =\sum_{i, \sigma}\left[\frac{U}{2} \hat{n}_{i}^{\sigma}\left(\hat{n}_{i}^{\sigma}-1\right)+\sum_{\langle j\rangle_{i}} \frac{U_{\mathrm{NN}}}{2} \hat{n}_{i}^{\sigma} \hat{n}_{j}^{\sigma}-\mu \hat{n}_{i}^{\sigma}\right] \\
& +W \sum_{i} \hat{n}_{i}^{a} \hat{n}_{i}^{b}-J \sum_{\langle i j\rangle}\left[\hat{a}_{i}^{\dagger} \hat{a}_{j}+\hat{b}_{i}^{\dagger} \hat{b}_{j}\right],
\end{aligned}
$$

where $\sigma=a, b$ indicates the two species (which in the specific case considered here are atoms in the lower and upper 2D optical lattice layer, respectively), $U$ is the on-site energy, $U_{\mathrm{NN}}$ the intralayer nearest neighbors repulsion, $W$ the interlayer attraction, $J$ the intralayer tunneling parameter, and $\mu$ the chemical potential, as schematically represented in Fig. 5.1. The parameters $U$ and $J$ are equal for the upper and lower layers and the chemical potentials $\mu$ are the same, since equal densities in the two layers are assumed. Notice that since $W<0$, it is necessary to have $U+W>0$ to avoid collapse. The symbols $\langle i j\rangle$ and $\langle j\rangle_{i}$ indicate nearest neighbors.

We focus on the physical situation in which the two layers are very close to one another, namely $d_{\perp} \ll d$, because in this limit particles at the same lattice site $i$ of different layers pair into composites. The composites localize in a MI 
state for small values of the tunneling coefficient, while for larger values of $J$ the pairs hop around in the optical lattice forming a Pair-superfluid (PSF) phase [65]. Furthermore, the presence of the long-range interactions leads to the formation of a novel pair-supersolid phase (PSS), namely, a supersolid of composites.

\subsubsection{Ground state and single-particle single-hole ex- citations}

We write the Hamiltonian (6.1) as $\hat{H}=\hat{H}_{0}+\hat{H}_{1}$, where

$$
\begin{aligned}
\hat{H}_{0} & =\sum_{i, \sigma}\left[\frac{U}{2} \hat{n}_{i}^{\sigma}\left(\hat{n}_{i}^{\sigma}-1\right)+\sum_{\langle j\rangle_{i}} \frac{U_{\mathrm{NN}}}{2} \hat{n}_{i}^{\sigma} \hat{n}_{j}^{\sigma}-\mu \hat{n}_{i}^{\sigma}\right]+W \sum_{i} \hat{n}_{i}^{a} \hat{n}_{i}^{b} \\
\hat{H}_{1} & =-J \sum_{\langle i j\rangle}\left[\hat{a}_{i}^{\dagger} \hat{a}_{j}+\hat{b}_{i}^{\dagger} \hat{b}_{j}\right]
\end{aligned}
$$

and we consider $\hat{H}_{1}$ to be a small perturbation on the interaction term (5.2). For any given classical configuration of atoms in the lattice given by $|\Phi\rangle=\prod_{i}\left|n_{i}^{a}, n_{i}^{b}\right\rangle$, we can use the perturbative mean field approach derived in Sec. 2.2.2 to analyze the stability of $|\Phi\rangle$ with respect to particle and hole excitations. The chemical potentials for the two species are the same, which fixes the same number of particles to be equal for both species, and since $W<0$, in the limit of close layers the system naturally tends to

minimize the differences in the number of particles between the upper and lower layer at the same lattice site. At $J=0$, this is easily understood if we write Hamiltonian (5.2) in terms of the sum and the difference operators,

$$
\begin{aligned}
\hat{m}_{i} & =\frac{\hat{n}_{i}^{a}+\hat{n}_{i}^{b}}{2} \\
\hat{s}_{i} & =\frac{\hat{n}_{i}^{a}-\hat{n}_{i}^{b}}{2},
\end{aligned}
$$

and write explicitly the expression for the expectation value of $\hat{H}_{0}$ on $|\Phi\rangle$, which is given by

$$
\begin{aligned}
\left\langle\Phi\left|\hat{H}_{0}\right| \Phi\right\rangle= & \sum_{i}\left[-(2 \mu+U) m_{i}+(U+W) m_{i}^{2}+U_{\mathrm{NN}} \sum_{\langle j\rangle_{i}} m_{i} m_{j}\right. \\
& \left.+(U-W) s_{i}^{2}+U_{\mathrm{NN}} \sum_{\langle j\rangle_{i}} s_{i} s_{j}\right],
\end{aligned}
$$


with $m_{i}=\left\langle\Phi\left|\hat{m}_{i}\right| \Phi\right\rangle$ and $s_{i}=\left\langle\Phi\left|\hat{s}_{i}\right| \Phi\right\rangle$. Since $W<0$, in the limit of $(U+W), U_{\mathrm{NN}} \ll U$ the energy above is minimized by setting $s_{i}=0$ and the density is fixed by $m_{i}$ at each site. We therefore restrict ourselves to study the stability of such states

$$
|\alpha\rangle=\prod_{i}\left|n_{i}, n_{i}\right\rangle_{i}
$$

with equal occupation of the two species $a$ and $b$ at each site, therefore a distribution of pairs composed by one atom of each species.

For any classical configuration (5.6), we can study the stability of $|\alpha\rangle$ with respect to particle and hole excitations using the same method derived in Sec. 2.2.2, and calculate the order parameter $\varphi_{i}=\left\langle\hat{a}_{i}\right\rangle=\left\langle\hat{b}_{i}\right\rangle$ at each lattice site, given by

$$
\varphi_{i}=J \bar{\varphi}_{i}\left[\frac{m_{i}+1}{E_{\mathrm{P}}^{i}}+\frac{m_{i}}{E_{\mathrm{H}}^{i}}\right],
$$

where $m_{i}=n_{i}^{a}=n_{i}^{b}$, and $\bar{\varphi}_{i}=\sum_{\langle j\rangle_{i}} \varphi_{i}$. The energy cost for a particle (P) and a hole $(\mathrm{H})$ excitation at the $i$-th site of the configuration $|\alpha\rangle$, are respectively given by

$$
\begin{aligned}
& E_{\mathrm{P}}^{i}=-\mu+U m_{i}+V_{\mathrm{dip}}^{1, i}+W m_{i} \\
& E_{\mathrm{H}}^{i}=\mu-U\left(m_{i}-1\right)-V_{\mathrm{dip}}^{1, i}-W m_{i},
\end{aligned}
$$

where we define $V_{\text {dip }}^{1, i}=U_{\mathrm{NN}} \sum_{\langle j\rangle_{i}} m_{j}$, as the in-plane dipolar interaction, i.e. the dipole-dipole interaction that experiences one atom positioned at site $i$ of the lattice, with the rest of the particles belonging to the same plane. Notice that the excitations (5.8), differ from the particle-hole excitations in a single layer (2.19), only because of the presence of the out-of-plane dipolar interaction $W m_{i}$. However we will see that the presence of $W$ is far from being trivial, in particular it does not only induce a shift in the excitation energies but is responsible for new and different physical behaviors with respect to the single layer model. For the evaluation of the order parameter (5.7) it is necessary to require the particle and hole excitations to be positive, which results in the equations

$$
U\left(m_{i}-1\right)+V_{\mathrm{dip}}^{1, i}+W m_{i}<\mu<U m_{i}+V_{\mathrm{dip}}^{1, i}+W m_{i} .
$$

One finds such an equation (5.7) and conditions (5.9) for each site of the lattice, and similarly as in Sec. 2.2.2, we write the coupled equations (5.7) in the matrix form $\mathcal{M}\left(\mu, U, J, U_{\mathrm{NN}}, W\right) \cdot \vec{\varphi}=0$, with $\vec{\varphi} \equiv\left(\cdots \varphi_{i} \cdots\right)$. Therefore, if the configuration $|\alpha\rangle$ is a local minimum with respect to the particle and hole 
excitations (5.8), for every $\mu$ the smallest $J$ for which $\operatorname{det}\left[\mathcal{M}\left(\mu, U, J, U_{\mathrm{NN}}, W\right)\right]=$ 0 gives the lobe boundary for the $|\alpha\rangle$ configuration in the $J$ vs. $\mu$ plane. We find that in the limit $(U+W) / U \rightarrow 0$, asymptotically all classical configurations $|\alpha\rangle$ develop an insulating lobe which tend to overlap one another as illustrated in Fig. 5.2 with blue thin lines.

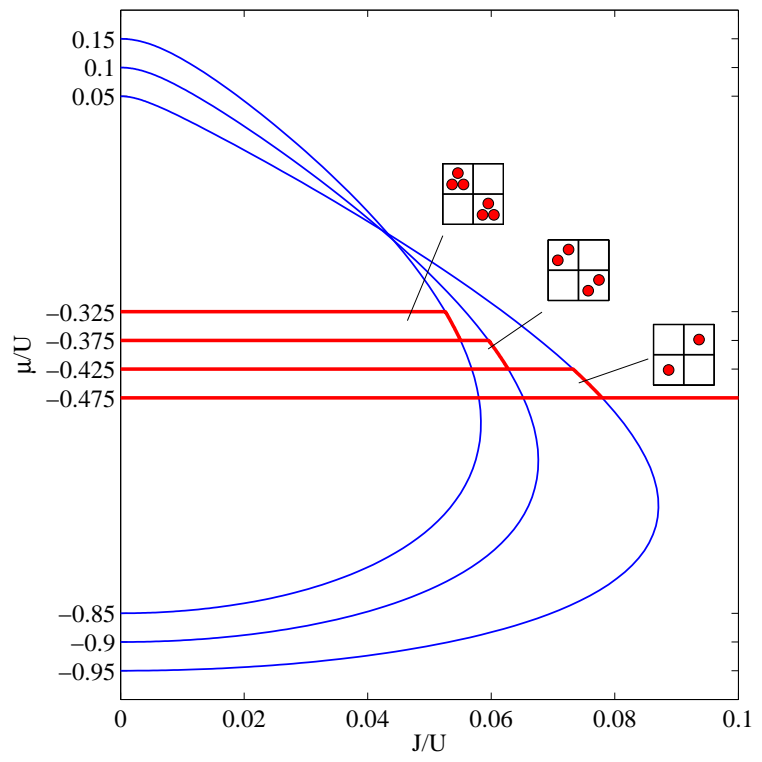

Figure 5.2: Insulating lobes calculated with respect to single-particle and single-hole excitations for all filling factors $\nu$, ranging from $\nu=0$ to $\nu=3$, of a $2 \times 2 \times 2$ elementary cell with periodic boundary conditions only in the directions perpendicular to the orientation of the dipoles (see Fig. 5.1). The lattice parameters are given by $U_{N N}=0.025 U$ and $W=-0.95 U$, which can be obtained for an inter-layer distance $d_{\perp}=0.37 d$. Notice the overlap of all the lobes which does not happen in a single layer situation.

This situation has to be compared to a single layer model in which not all classical distributions have an insulating lobe but only some configurations at some specific filling factors $\nu$, and the ground state lobes do not overlap. In this situation, in order to find the ground state one has to compare the energy of the insulating configurations at each value of the chemical potential $\mu$. The configuration with the lowest energy is then the ground state, as illustrated in Fig. 5.2 with the red lobes along with a sketch of the ground state configurations in the panels. Except for the filling factor zero $\nu=$ 
$\frac{2}{N_{S}} \sum_{i} m_{i}=0$, which is found to be below the horizontal red line at $\mu=$ $-0.475 U$ ( $N_{S}$ is the total number of sites), the ground state is a multiple of the fractional filling factor $\nu=1 / 2$ with a spatial density distribution of a checkerboard, doubly occupied checkerboard and so on depending on the value of the chemical potential $\mu$.

\subsection{Low-energy subspace and effective Hamil- tonian}

The reason behind this overlapping lobes lays in the fact that the lowest lying excitations are not of single-particle-hole of Eq. (5.8), but are rather obtained by adding or removing two particles at a given site of the $|\alpha\rangle$ configurations. In fact, in the limit of parameters we are considering here, all the density distributions (5.6) span a low-energy subspace which is energetically well separated from the rest of the Hilbert space, therefore the description of such a system, as well as its excitations, can be done within a low-energy theory restricted to an effective Hamiltonian $\hat{H}_{\text {eff }}$ acting only on the subspace spanned by the $|\alpha\rangle$-s. The subspace is described by all classical distributions of atoms in the lattice $|\alpha\rangle$ of Eq. (5.6) with equal occupation of the two species $a$ and $b$ at each site, therefore a distribution of pairs composed by one atom of each species. In such a situation there are two types of processes that depend on the time scale we are looking at the system [79]: (i) the slow processes which drive the system through different states (energetically very close to one another) of the low-energy subspace, and (ii) the fast processes which couple the low energy subspace with the rest of the Hilbert space composed of high-energy states. The latter are called virtual subspace and are coupled to the low-energy subspace through the tunneling Hamiltonian $\hat{H}_{1}$ of Eq. (5.3) via single particle hopping. The relevant virtual subspace is obtained from the states $|\alpha\rangle$ by breaking one composite, namely

$$
\begin{aligned}
\left|\gamma_{i j}^{(a)}\right\rangle & =\frac{\hat{a}_{i}^{\dagger} \hat{a}_{j}}{\sqrt{n_{j}\left(n_{i}+1\right)}}|\alpha\rangle \\
\left|\gamma_{i j}^{(b)}\right\rangle & =\frac{\hat{b}_{i}^{\dagger} \hat{b}_{j}}{\sqrt{n_{j}\left(n_{i}+1\right)}}|\alpha\rangle,
\end{aligned}
$$

as schematically represented in Fig. 6.3 for a uniform distribution $|\alpha\rangle$ of one atom per site. All other states are not coupled to $|\alpha\rangle$ via single particle hopping and hence do not contribute. 

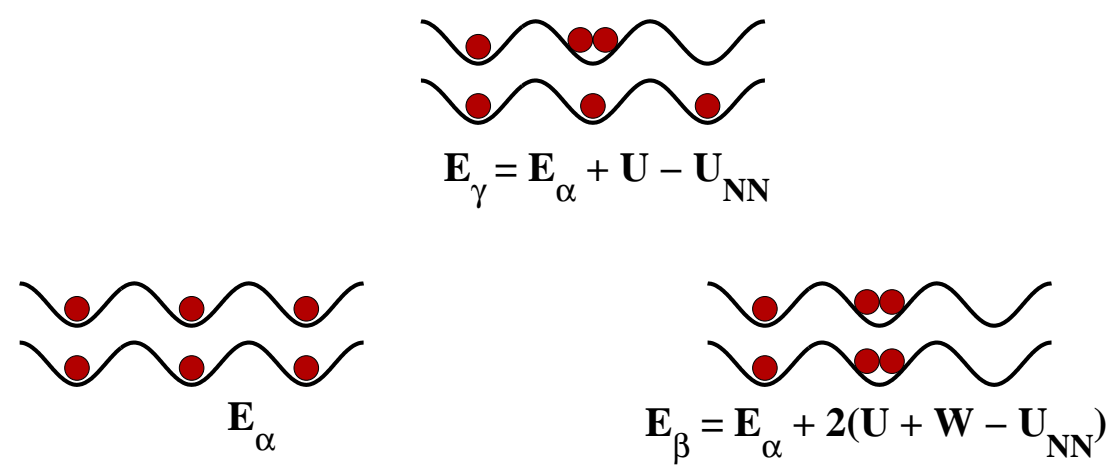

Figure 5.3: Schematic representation of the low-energy subspace. The state $|\alpha\rangle$ is uniformly occupied by one particle per site and its unperturbed energy, i.e. at $J=0$, is given by $E_{\alpha}$. The states $|\alpha\rangle$ and $|\beta\rangle$ (of unperturbed energy $E_{\beta}$ ) belong to the subspace, they are connected through a second order process in the tunneling via the the state $|\gamma\rangle$ (of unperturbed energy $E_{\gamma}$ ), which belong to the virtual subspace. It is straightforward to notice that in the limit of $(U+W), U_{\mathrm{NN}} \ll U$, the energies above satisfy the necessary condition $E_{\alpha}, E_{\beta} \ll E_{\gamma}$ for the existence of the subspace.

The energy difference between the virtual states $|\gamma\rangle$, of energy $E_{\gamma}$, and the states $|\alpha\rangle$, of energy $E_{\alpha}$, is given by the sum of single particle plus single hole excitation energies of the states $|\alpha\rangle$, which is of the order of $U$ at $J=0$, and is minimized by the width of the lobes $|\alpha\rangle$ (see, e.g., Fig. 5.2) at finite $J$.

Slow processes drive the system through different states of the low energy subspace, $|\alpha\rangle$ and $|\beta\rangle$, via second order tunneling; this happens through a fast coupling with the virtual subspace. Since we are interested in the long time physics of the system, we have to average out all the fast processes and therefore we write an effective Hamiltonian in the subspace of pairs, and include tunneling through second order perturbation theory $[77,78]$. In the pair-state basis, the matrix elements of such a Hamiltonian in second order perturbation theory are given by

$$
\begin{aligned}
\left\langle\alpha\left|\hat{H}_{\mathrm{eff}}\right| \beta\right\rangle= & \left\langle\alpha\left|\hat{H}_{0}\right| \beta\right\rangle-\frac{1}{2} \sum_{\gamma}\left\langle\alpha\left|\hat{H}_{1}\right| \gamma\right\rangle\left\langle\gamma\left|\hat{H}_{1}\right| \alpha\right\rangle \\
& \times\left[\frac{1}{E_{\gamma}-E_{\alpha}}+\frac{1}{E_{\gamma}-E_{\beta}}\right]
\end{aligned}
$$

where $\hat{H}_{0}$, given by the interaction terms (5.2), is diagonal on the states $|\alpha\rangle$, and the single-particle tunneling term $\hat{H}_{1}$ in Eq. (5.3) is treated at second 
order. For a given state $|\alpha\rangle$,

$$
E_{\gamma_{i j}}-E_{\alpha}=U+(U+W)\left(m_{i}-m_{j}\right)+U_{\mathrm{NN}} \Delta m_{\mathrm{NN}}^{i j}
$$

with $\Delta m_{\mathrm{NN}}^{i j}=\sum_{\langle k\rangle_{i}} m_{k}-\sum_{\langle k\rangle_{j}} m_{k}-1$, where $m_{i}$ indicates the pair occupation number at site $i$ as defined in Eq. (5.4). For $\mathrm{U}+\mathrm{W}, U_{\mathrm{NN}} \ll U$, the denominators $E_{\gamma_{i j}}-E_{\alpha}$ are all of order $U$, which leads to

$$
\hat{H}_{\mathrm{eff}}^{(0)}=\hat{H}_{0}-\frac{2 J^{2}}{U} \sum_{\langle i j\rangle}\left[\hat{m}_{i}\left(\hat{m}_{j}+1\right)+\hat{c}_{i}^{\dagger} \hat{c}_{j}\right],
$$

where $\hat{c}_{i}$ and $\hat{c}_{i}^{\dagger}$ are the pair destruction and creation operators such that

$$
\begin{aligned}
\hat{c}_{i}\left|m_{i}\right\rangle & =m_{i}\left|m_{i}-1\right\rangle \\
\hat{c}_{i}^{\dagger}\left|m_{i}\right\rangle & =\left(m_{i}+1\right)\left|m_{i}+1\right\rangle .
\end{aligned}
$$

One can easily obtain corrections to $\hat{H}_{\text {eff }}^{(0)}$ by expanding (5.12) at higher orders in $(U+W) / U$ and $U_{\mathrm{NN}} / U$ but, as we will see, the zeroth order is already quite accurate to describe the physics of the system for the range of parameters we consider.

\subsubsection{Ground state insulating phases and two-particle two-hole excitations}

We now make use of the effective Hamiltonian $\hat{H}_{\text {eff }}^{(0)}$ derived above to study the ground state phase diagram of the system, starting from the insulating states. For every classical distribution of pairs in the lattice we can calculate the pair order parameter $\psi_{i}=\left\langle\hat{c}_{i}\right\rangle$ with the perturbative mean-field method derived in Sec. 2.2.2, and get the expression

$$
\psi_{i}=\frac{2 J^{2}}{U}\left[\frac{\left(m_{i}+1\right)^{2}}{E_{2 \mathrm{P}}^{i}(J)}+\frac{m_{i}^{2}}{E_{2 \mathrm{H}}^{i}(J)}\right] \bar{\psi}_{i},
$$

where $\bar{\psi}_{i}=\sum_{\langle j\rangle_{i}} \psi_{j}$, and the energy costs of adding a pair (2P) and removing a pair $(2 \mathrm{H})$ can be calculated with the diagonal part of Eq. (5.13), and are respectively given by

$$
\begin{aligned}
& E_{2 \mathrm{P}}^{i}(J)=-2 \mu+2 U m_{i}+\left(2 m_{i}+1\right) W+2 V_{\mathrm{dip}}^{1, i}-\frac{2 J^{2}}{U} \sum_{\langle k\rangle_{i}}\left(2 m_{k}+1\right) \\
& E_{2 \mathrm{H}}^{i}(J)=2 \mu-2 U\left(m_{i}-1\right)-\left(2 m_{i}-1\right) W-2 V_{\mathrm{dip}}^{1, i}+\frac{2 J^{2}}{U} \sum_{\langle k\rangle_{i}}\left(2 m_{k}+1\right),
\end{aligned}
$$


with $V_{\text {dip }}^{1, i}$ defined just after Eq. (5.8). By imposing the positivity of these excitations we get to the expressions

$$
\begin{array}{r}
U\left(m_{i}-1\right)+\left(m_{i}-\frac{1}{2}\right) W+V_{\mathrm{dip}}^{1, i}-\frac{J^{2}}{U} \sum_{\langle k\rangle_{i}}\left(2 m_{k}+1\right)<\mu< \\
U m_{i}+\left(m_{i}+\frac{1}{2}\right) W+V_{\mathrm{dip}}^{1, i}-\frac{J^{2}}{U} \sum_{\langle k\rangle_{i}}\left(2 m_{k}+1\right),
\end{array}
$$

which can be easily compared with Eqs. (5.9) for the single-particle singlehole excitations. At $J=0$, it is straightforward to notice that for any $W<0$ the two-particles two-holes excitations of Eqs. (5.17) give more restrictive conditions than their corresponding single particle-hole excitations of Eqs. (5.9). Therefore we conclude that at $J=0$, for any $W<0$ the low-lying excitations of a classical distribution of pairs in the lattice are obtained by adding or removing a pair at any site $i$, in agreement with the previous statements.

Using Eq. (5.15) and conditions (5.17), one can calculate the mean-field lobes for any given configuration of pairs in the lattice. The lobes for the checkerboard and doubly occupied checkerboard are shown in Fig. 5.4 for the 0th (full lines) and 1st order (dashed lines) effective Hamiltonians. The comparison between the two shows that, for the parameters considered here, the 0th order already captures the physics accurately. It is worth noticing that the $J^{2}$ dependence of the energy of the elementary excitations is at the origin of the reentrant behavior of the lobes, which was predicted by exact matrix-product-state calculations for the 1D geometry in [65]

\subsection{Gutzwiller mean-field approach and va- lidity of the low energy subspace}

While the $M I$ phases are predictable through the perturbative mean-field approach of Eqs. (5.15) for the pair order parameters, to identify the $S F$ phases, both $P S F$ and $P S S$ outside of the lobes, it is necessary to make use of the imaginary time evolution introduced in Sec. 2.2.1 based on a Gutzwiller Ansatz for the pair wave function. Therefore, we need to calculate the dynamics equation equivalent to (2.6) in the low energy subspace described by the effective Hamiltonian $\hat{H}_{\mathrm{eff}}^{(0)}$ of Eq. (5.13).

We begin with the time dependent Gutzwiller wave function for the pairs, which is given by

$$
|\Phi\rangle=\prod_{i} \sum_{m} f_{\mathrm{m}}^{(i)}|m\rangle_{i}
$$




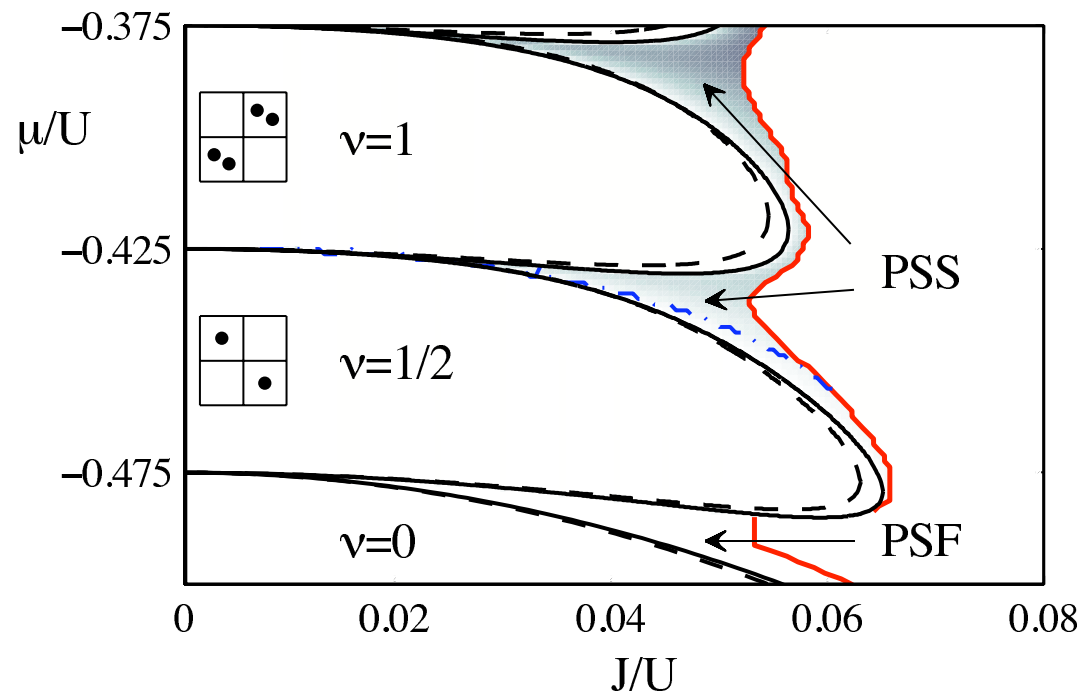

Figure 5.4: Phase diagram of the effective Hamiltonian, with $U_{\mathrm{NN}}=0.025 U$, $W=-0.95 U$, which can be obtained for $d_{\perp}=0.37 d$. The black full lines are the semi-analytic solution of Eq.(5.15) indicating the boundaries of the insulating lobes for the checkerboard $(\nu=1 / 2)$ and the doubly occupied checkerboard $(\nu=1)$. The black dashed lines are the boundaries of the insulating lobes for 1st order expansion of $\hat{H}_{\text {eff }}$. The shaded area is the PSS phase predicted by the Gutzwiller approach. The red line indicates the estimated limit of validity of $\hat{H}_{\text {eff }}^{(0)}$ (see text).

where we allow the Gutzwiller amplitudes to depend on time $f_{\mathrm{m}}^{(i)}(t)$. The order parameter is readily obtained from (5.18) as

$$
\psi_{i}=\left\langle\Phi\left|\hat{c}_{i}\right| \Phi\right\rangle=\sum_{m}(m+1) f_{\mathrm{m}}^{*(i)} f_{\mathrm{m}+1}^{(i)} .
$$

As in Sec. 2.2.1, we obtain the equations of motion for the amplitudes by minimizing the action of the system, given by $S=\int \mathrm{d} t \mathcal{L}$, with respect to the variational parameters $f_{\mathrm{m}}^{(i)}(t)$ and their complex conjugates $f_{\mathrm{m}}^{*(i)}(t)$, where

$$
\mathcal{L}=\frac{\langle\Phi \mid \dot{\Phi}\rangle-\langle\dot{\Phi} \mid \Phi\rangle}{2 i}-\left\langle\Phi\left|\hat{H}_{\mathrm{eff}}^{(0)}\right| \Phi\right\rangle,
$$

is the Lagrangian of the system in the quantum state $|\Phi\rangle[60]$, and $|\dot{\Phi}\rangle$ is the time derivative of the wave function (5.18). Therefore equating to zero the 
variation of the action with respect to $f_{\mathrm{m}}^{*(i)}$ leads to the equations

$$
\begin{aligned}
i \hbar \frac{\mathrm{d}}{\mathrm{d} t} f_{\mathrm{m}}^{(i)}= & -\frac{2 J^{2}}{U}\left[\bar{\psi}_{i} m f_{\mathrm{m}-1}^{(i)}+\bar{\psi}_{i}^{*}(m+1) f_{\mathrm{m}+1}^{(i)}\right]+[U m(m-1)-2 \mu m \\
& \left.+2\left(U_{N N}-\frac{2 J^{2}}{U}\right) m \sum_{\langle j\rangle_{i}}\left\langle\hat{m}_{j}\right\rangle-\frac{2 J^{2}}{U} z m\right] f_{\mathrm{m}}^{(i)}
\end{aligned}
$$

where $\left\langle\hat{m}_{i}\right\rangle=\sum_{m} m\left|f_{\mathrm{m}}^{(i)}\right|^{2}$, the fields $\bar{\psi}_{i}=\sum_{\langle j\rangle_{i}} \psi_{j}$ and $\sum_{\langle j\rangle_{i}}\left\langle\hat{m}_{j}\right\rangle$, have to be calculated self-consistently as explained in Sec. 2.2.1, and $z=\sum_{\langle j\rangle_{i}} 1=4$ is the coordination number in each lattice layer. One can write the set of coupled equations (5.21) in the matrix form

$$
i \hbar \frac{\mathrm{d}}{\mathrm{d} t} \vec{f}=\mathcal{M}\left[\vec{f}, \mu, U, J, U_{N N}\right] \cdot \vec{f}
$$

where $\vec{f}=\left[f_{0}^{(1)}, f_{1}^{(1)}, \cdots, f_{\mathrm{m}}^{(i)}, \cdots f_{\mathrm{m}_{\max }}^{\left(N_{S}\right)}\right]^{\mathrm{T}}$, is the vector of the Gutzwiller amplitudes and $N_{S}$ the total number of lattice sites. The solution of Eq. (5.22) can be easily obtained numerically with the same procedure explained in Sec. 2.2.1, and by making use of the imaginary time evolution we get the ground state phase diagram of Fig. (5.4).

To get reliable results, one should combine the Gutzwiller predictions with an estimate of the limits of validity of $\hat{H}_{\mathrm{eff}}^{(0)}$, beyond which the subspace of pairs looses its meaning. Before starting the discussion on the validity of the subspace, let us explain how we define the dominant classical configurations of a given state $|\Phi\rangle$. It is not difficult to see that Eq. (5.18) can be equivalently written as

$$
|\Phi\rangle=\sum_{\{\vec{m}\}} g_{\vec{m}} \prod_{i}\left|m_{i}\right\rangle_{i},
$$

where $\vec{m}=\left(m_{1}, \ldots, m_{i}, \ldots, m_{\mathrm{N}_{\mathrm{S}}}\right)$ is a collection of the indices $m$ at each site, and we have introduced the notation such that $g_{\vec{m}}=\prod_{i} f_{\mathrm{m}_{\mathrm{i}}}^{(i)}$. The advantage of writing the Gutzwiller state $|\Phi\rangle$ in the form (5.23), lays in the product over single-site Fock state $|\alpha\rangle=\prod_{i}\left|m_{i}\right\rangle_{i}$, which is nothing but a classical distribution of atoms in the lattice. Therefore we can rewrite Eq. (5.23) as

$$
|\Phi\rangle=\sum_{\{\alpha\}} g_{\alpha}|\alpha\rangle
$$

For each point of the phase diagram, from the ground state Gutzwiller wavefunction, we define the dominant classical configurations with the criteria $\left|g_{\vec{m}}\right|=\left|\prod_{i} f_{\mathrm{m}_{\mathrm{i}}}^{(i)}\right|>0.02$, and we require $\left|f_{\mathrm{m}_{\mathrm{i}}}^{(i)}\right|^{2}>0.05$, implying that each of the contributing $f_{\mathrm{m}_{\mathrm{i}}}^{(i)}$ should also be sufficiently large. For each of these 
configurations, we calculate the lobe with respect to single particle-hole excitations $^{2}$. If the system at this given point of the phase diagram turns out to be stable against all dominant single particle-hole excitations (in other words, if this point is inside all selected single particle-hole lobes), $\hat{H}_{\text {eff }}^{(0)}$ is considered valid at that point. This procedure is shown for $J=0.05 U$ and $\mu=-0.4375 U$ in Fig. 5.5, and gives the red line of Fig. (5.4). On the right hand side of this red line, the low energy subspace is not well defined and therefore the effective Hamiltonian looses its meaning, leaving the description of the system to the domain of single-particle single-hole excitation theory that predicts $S F$ and $S S$ phases for each component separately.

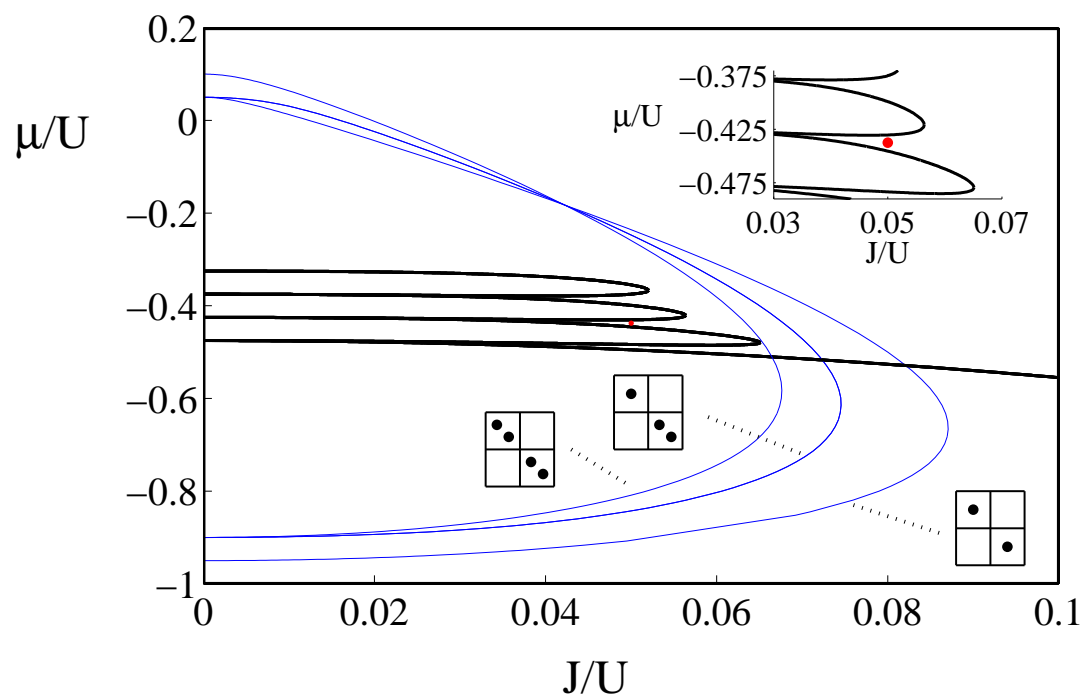

Figure 5.5: Pair insulating lobes for $\nu=0,1 / 2,1,3 / 2$ (thick lines); Lobes with respect to single particle-hole excitations (thin blue lines) for the dominant configurations in the ground state at $J=0.05 U$ and $\mu=-0.4375 U$, namely $m_{i}=0$ and $m_{j}=1,2$ (for $i, j$ nearest neighboring sites). The inset shows a zoom of the pair phase diagram.

To summarize, in the ground state phase diagram of Fig. 5.4 we identify four different types of phases characterized by different values of the order parameters, the single-particle order parameters for each species $\varphi_{i}^{a}=\left\langle\hat{a}_{i}\right\rangle$, $\varphi_{i}^{b}=\left\langle\hat{b}_{i}\right\rangle$, and the pair order parameter $\psi_{i}=\left\langle\hat{c}_{i}\right\rangle$.

\footnotetext{
${ }^{2}$ We have checked that the validity region is not strongly modified upon small changes in these conditions.
} 
1. The Mott insulating checkerboard phases (MI) characterized by fractional filling factors $\nu$ and vanishing order parameters $\varphi_{i}^{a}=\varphi_{i}^{b}=\psi_{i}=0$ inside the lobes.

2. The pair-superfluid phase (PSF) in which the single particle order parameters are zero $\varphi_{i}^{a}=\varphi_{i}^{b}=0$, while the pair order parameter is uniformly non-zero $\psi_{i} \neq 0$, signaling a finite fraction of superfluid density of the pairs.

3. The pair-supersolid (PSS) characterized by vanishing single-particle order parameters $\varphi_{i}^{a}=\varphi_{i}^{b}=0$, and non vanishing pair order parameter $\psi_{i} \neq 0$, coexisting with broken translational symmetry, namely, a modulation of both density and order parameter on a scale larger than the one of the lattice spacing, analogously to the supersolid phase.

4. We infer superfluid and supersolid phases of both components $S F_{a}$ and $S F_{b}\left(S S_{a}\right.$ and $S S_{b}$ respectively) with non vanishing single species order parameters $\varphi_{i}^{a} \neq 0$ and $\varphi_{i}^{b} \neq 0$, as well as non vanishing $\psi_{i} \neq 0$, on the right hand side of the red line of Fig. 5.4, which we estimate to be the limit of validity of the low energy subspace for the parameters considered here. 


\section{Chapter 6}

\section{Counterflow Supersolid of anti-polarized dipolar Bosons in a $2 \mathrm{D}$ optical lattice}

\subsection{Introduction}

In this part we present a work which is still on going. The system we are currently studying has many similarities to the case of the 2D layers we have already discussed, and it is interesting not only from a theoretical point of view, but also because of its possible experimental realizations. Recently, magnetic confinement of neutral, ground state $\mathrm{OH}$ molecules, has been reported $[75,76]$. OH, which is Hund's case A [75], features both an electric and a magnetic moment. While, the magnetic spins can always be aligned by optical pumping, the electric dipole orientation can be controlled via RF pulses, and, in particular it can be aligned or anti-aligned with the magnetic moment.

\subsection{Hamiltonian of the system}

We consider a sample of polarized dipoles in the presence of a $2 \mathrm{D}$ optical lattice, and an extra confinement in the perpendicular direction. The dipoles are free to point in both directions perpendicularly to the plane, which results in a nearest neighbor interaction that is repulsive for aligned dipoles, while attractive for anti-aligned, as shown in Fig. 6.1. This situation can be obtained with $\mathrm{OH}$ molecules as explained previously. 
The system is described by the Hamiltonian

$$
\begin{aligned}
\hat{H} & =\sum_{i, \sigma}\left[\frac{U_{\sigma}}{2} \hat{n}_{i}^{\sigma}\left(\hat{n}_{i}^{\sigma}-1\right)+\frac{U_{\sigma \sigma^{\prime}}}{2} \hat{n}_{i}^{\sigma} \hat{n}_{i}^{\sigma^{\prime}}-\mu_{\sigma} \hat{n}_{i}^{\sigma}\right] \\
& +\sum_{\langle i j\rangle_{\sigma}} \frac{U_{N N}}{2}\left[\hat{n}_{i}^{\sigma} \hat{n}_{j}^{\sigma}-\hat{n}_{i}^{\sigma} \hat{n}_{j}^{\sigma^{\prime}}\right]-J \sum_{\langle i j\rangle}\left[\hat{a}_{i}^{\dagger} \hat{a}_{j}+\hat{b}_{i}^{\dagger} \hat{b}_{j}\right],
\end{aligned}
$$

where $\sigma=a, b$ indicates the two species (which in the specific case considered here are dipoles pointing in the up and down direction perpendicular to the $2 \mathrm{D}$ plane of the lattice, respectively), $U_{a a}$ and $U_{b b}$ are the on-site energies for particles of the same species, while $U_{a b}$ is the on-site energy for different species. The nearest neighbor dipolar interaction is attractive (repulsive) for particles of the same (different) species, with strength $U_{N N}>0$ (or respectively $\left.-U_{N N}\right)$, the tunneling coefficient $J$ is equal for both the species, while the densities of the dipoles pointing upwards and downwards are fixed by the corresponding chemical potentials $\mu_{\sigma}$.

The on-site interactions have two contributions: one is arising from the s-wave scattering length $U_{s}=\frac{4 \pi \hbar^{2} a_{s}}{m} \int \mathrm{d}^{3} r \rho(\mathbf{r})^{2}$, and the second one is due to the onsite dipole-dipole interaction $U_{\mathrm{dd}}=\frac{1}{(2 \pi)^{3}} \int \mathrm{d}^{3} k \widetilde{U_{\mathrm{dd}}}(\mathbf{k}) \widetilde{\rho}^{2}(\mathbf{k})$, where $\widetilde{U_{\mathrm{dd}}}(\mathbf{k})$ and $\widetilde{\rho}^{2}(\mathbf{k})$ are the Fourier transform of the dipole potential (1.28) and the density respectively. In the system we consider, the s-wave scattering length is independent of the orientation of the dipoles. Instead, the on-site dipolar contribution $U_{\mathrm{dd}}$ depends both on the orientation of the dipoles and on the geometry of the trapping potential, and it can be varied by changing the ratio between the vertical to the axial confinement as discussed in Sec. 1.3.2. For simplicity we will focus on the specific case of a spherically symmetric confinement, where the on-site dipolar interactions average out to zero $U_{\mathrm{dd}}=$ 0 , as discussed in Sec. 1.3.2, and the resulting on-site interactions are all equal to $U$.

\subsubsection{Filling factor and imbalance}

The properties of the system are better understood in terms of the operators given by the sum (filling factor) and the difference (imbalance) of the two species at each site of the lattice, respectively given by

$$
\begin{aligned}
\hat{\nu}_{i} & =\frac{\hat{n}_{i}^{a}+\hat{n}_{i}^{b}}{2} \\
\hat{m}_{i} & =\frac{\hat{n}_{i}^{a}-\hat{n}_{i}^{b}}{2},
\end{aligned}
$$




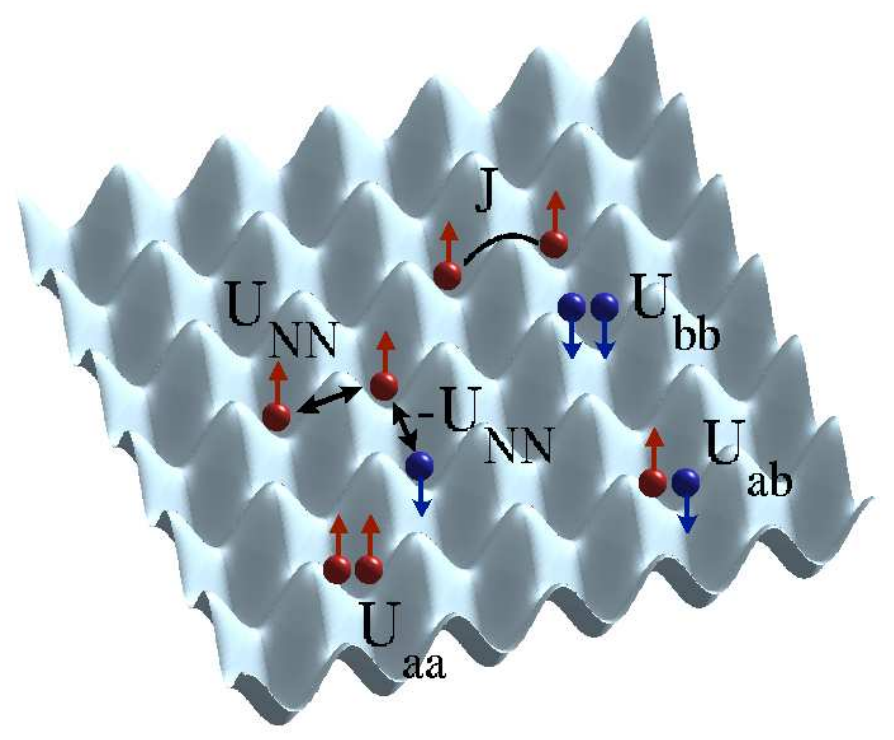

Figure 6.1: Schematic representation of a 2D optical lattice populated with dipolar Bosons polarized in both directions perpendicular to the lattice plane. The particles feel repulsive intra-species $U_{a a}, U_{b b}$, and inter-species $U_{a b}$ repulsive on-site energies. The nearest-neighbor interaction is repulsive $U_{\mathrm{NN}}>0$ for aligned dipoles, while it is attractive $-U_{\mathrm{NN}}$ for anti-aligned particles, and the hopping term $J$ is equal for both the species.

which are simultaneously diagonal on a given Fock state $|\nu, m\rangle_{i}$. Notice that the eigenvalues of these two operators are not independent. In fact by fixing $\nu$, the eigenvalues of $\hat{m}_{i}$ can only assume $2 \nu+1$ values given by $m=$ $\{-\nu,-\nu+1, \ldots,+\nu\}$, in complete analogy with the spin angular momentum operator $\hat{S}_{i}^{2}$ and its projection along the $z$ axis $\hat{S}_{i}^{z}$, as we will discuss in Sec. 6.2.2. It is useful to introduce the average magnetization of the system, defined as

$$
M=\frac{1}{N_{\mathrm{S}}} \sum_{i} m_{i},
$$

where $N_{\mathrm{S}}$ is the total number of lattice sites, because, as we will see, it is a convenient quantity to describe the phases of the system.

We substitute Eqs. (6.2) into Hamiltonian (6.1), which we write as $\hat{H}=$ 
$\hat{H}_{0}^{\nu}+\hat{H}_{0}^{m}+\hat{H}_{1}^{\nu m}$, where the different terms read

$$
\begin{aligned}
\hat{H}_{0}^{\nu} & =\sum_{i}\left[-2 \mu_{+} \hat{\nu}_{i}+2 U \hat{\nu}_{i}\left(\hat{\nu}_{i}-\frac{1}{2}\right)\right] \\
\hat{H}_{0}^{m} & =\sum_{i}\left[-2 \mu_{-} \hat{m}_{i}+2 U_{N N} \sum_{\langle j\rangle_{i}} \hat{m}_{i} \hat{m}_{j}\right] \\
\hat{H}_{1}^{\nu m} & =-J \sum_{\langle i j\rangle}\left[\hat{a}_{i}^{\dagger} \hat{a}_{j}+\hat{b}_{i}^{\dagger} \hat{b}_{j}\right] .
\end{aligned}
$$

We have introduced the chemical potentials

$$
\mu_{ \pm}=\frac{\mu_{a} \pm \mu_{b}}{2}
$$

which respectively fix the eigenvalues of the filling factor and the imbalance operators (6.2). In the following we will consider $\hat{H}_{1}^{\nu m}$ to be a small perturbation on the interaction terms. In the limit of $U \gg U_{N N}, J$, the ground state of the system is found to be a uniform distribution of constant filling factor $\nu_{i}=\bar{\nu}$ at each site of the lattice. The value of $\bar{\nu}$ is fixed by $\mu_{+}$, and can be integer as well as semi-integer. This is better understood at $J=0$, where we can calculate the expectation value of $\hat{H}_{0}^{\nu}$ on a given classical distribution of atoms in the lattice $|\Phi\rangle=\prod_{i}\left|\nu_{i}, m_{i}\right\rangle_{i}$, as follows

$$
\left\langle\Phi\left|\hat{H}_{0}^{\nu}\right| \Phi\right\rangle=\sum_{i}\left[-2 \mu_{+} \nu_{i}+2 U \nu_{i}\left(\nu_{i}-\frac{1}{2}\right)\right],
$$

where $\nu_{i}=\left\langle\Phi\left|\hat{\nu}_{i}\right| \Phi\right\rangle$. In the energy (6.8) each site $i$ is self-similar, and like in the homogeneous case of a Bose-Hubbard Hamiltonian at $J=0$, the minimum of Eq. (6.8) is provided by a uniform distribution $\nu_{i}=\bar{\nu}$ at each site of the lattice. Instead, for a given $\bar{\nu}$, finding the magnetization which minimize the expectation value

$$
\left\langle\Phi\left|\hat{H}_{0}^{m}\right| \Phi\right\rangle=\sum_{i}\left[-2 \mu_{-} m_{i}+2 U_{N N} \sum_{\langle j\rangle_{i}} m_{i} m_{j}\right],
$$

is non-trivial due to the presence of the nearest neighbor repulsion $2 U_{\mathrm{NN}}$, where $m_{i}=\left\langle\Phi\left|\hat{m}_{i}\right| \Phi\right\rangle$. However, we can qualitatively argue that for $\left|\mu_{-}\right| \gg$ $U_{N N}$, the minimum of the energy (6.9) is obtained for $m_{i}=\nu \times \operatorname{sign}\left[\mu_{-}\right], \forall i$, which corresponds to a ferromagnetic phase (FM) of average magnetization $M=\nu \times \operatorname{sign}\left[\mu_{-}\right]$, where only particles of one species are present. Instead, for $\mu_{-}=0$, a succession of nearest neighbors $m_{i}=\nu$ and $m_{j}=-\nu$ provides the minimum of Eq. (6.9), and the phase is anti-ferromagnetic (AM), i.e. $M=0$. 
The spatial distribution of the particles is given by sites occupied from the species $a$ alternated with sites occupied by the species $b$ in a checkerboardlike structure. In Fig. 6.2 we plot the ground state at $J=0$, in the $\mu_{-}$vs. $\mu_{+}$ plane, where the text in parenthesis $(\nu, M)$ indicate respectively the filling factor and the average magnetization.

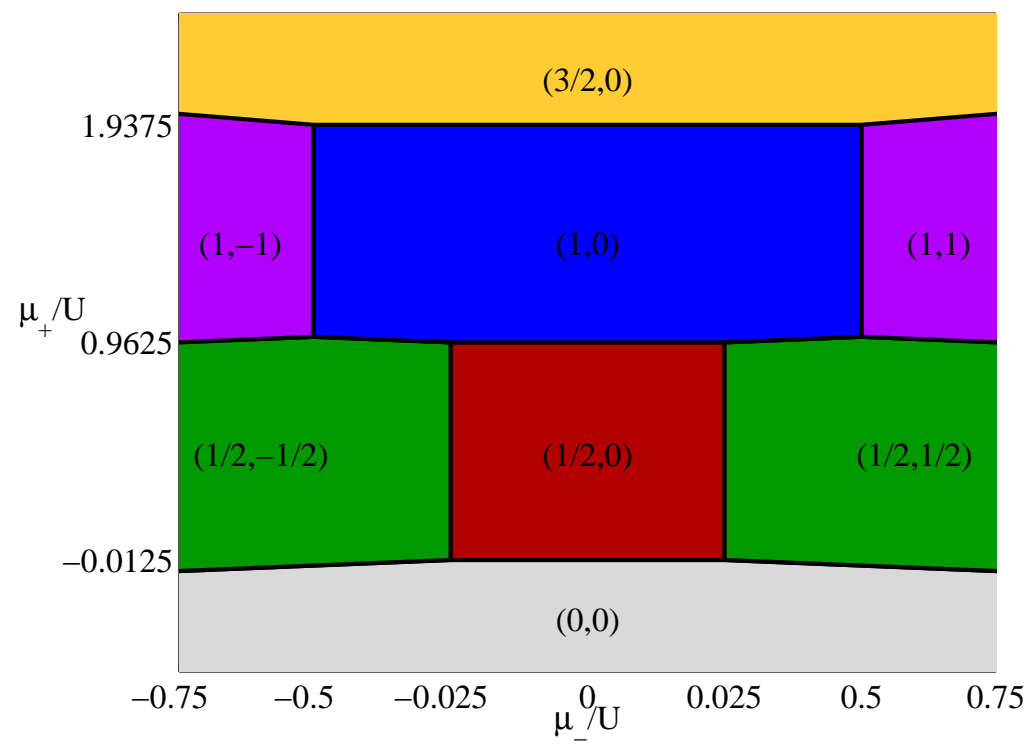

Figure 6.2: Ground state of the effective Hamiltonian $\hat{H}_{\text {eff }}^{(0)}$ (see text) at $J=0$, calculated for a $2 \times 2$ elementary cell satisfying periodic boundary conditions. The text in parenthesis $(\nu, M)$ indicate the filling factor $\nu$ and the average magnetization $M$, respectively.

In the next section we will include the presence of tunneling. In this situation we will see that the theoretical description of the system cannot be based on standard mean-field theory, which is not suitable to describe the ground state of the system in a correct way. In fact, the phase diagram of Fig. 6.2 has been obtained using an effective Hamiltonian, which describes correctly the physics of the system as we will explain in the following section.

\subsubsection{Low-energy subspace and effective Hamiltonian}

The ground state of the system at $J=0$ is described by a product over single-site Fock states of the type

$$
|\alpha\rangle=\prod_{i}\left|\nu, m_{i}\right\rangle_{i}
$$


with uniform on-site occupation $\nu$. A single particle hopping changes the total on-site population at the sites involved in the hopping process, and therefore breaks the translational invariance of the ground state with respect to the on-site occupation $\nu$. The energy cost of these excitations is of the order of the on-site interaction energy $U$, and is therefore very costly in the limit of $U \gg U_{N N}, J$. On the contrary, exchanging two particles from nearest neighboring sites or flipping the direction of one dipole (from up to down or vice versa), does not require such a large amount of energy. This defines a low-energy subspace spanned by the $|\alpha\rangle$ distributions of constant filling factor $\nu$ of Eq. (6.10), which is energetically well separated from the rest of the Hilbert space in the limit of parameters we are considering here. Thus, a successful description of such a system is obtained through an effective Hamiltonian $\hat{H}_{\text {eff }}$ restricted to the low-energy subspace, where single-particle hopping is suppressed and tunneling is included at second order perturbation theory. The validity of the effective Hamiltonian relies on the existence of this low-energy subspace well separated in energy from the subspace of virtual excitations, to which it is coupled via single-particle hopping. The relevant virtual subspace is then obtained from the states $|\alpha\rangle$ via single particle hopping, namely

$$
\begin{aligned}
\left|\gamma_{i j}^{(a)}\right\rangle & =\frac{\hat{a}_{i}^{\dagger} \hat{a}_{j}}{\sqrt{n_{j}^{a}\left(n_{i}^{a}+1\right)}}|\alpha\rangle \\
\left|\gamma_{i j}^{(b)}\right\rangle & =\frac{\hat{b}_{i}^{\dagger} \hat{b}_{j}}{\sqrt{n_{j}^{b}\left(n_{i}^{b}+1\right)}}|\alpha\rangle,
\end{aligned}
$$

as schematically represented in Fig. 6.3.

This situation is qualitatively not different than the one discussed in Sec. 5.2 in the context of the two layers, and therefore we can apply the same technique to compute $\hat{H}_{\text {eff }}$. In the basis of constant on-site population $\nu$, the matrix elements of such a Hamiltonian in second order perturbation theory are given by

$$
\begin{aligned}
\left\langle\alpha\left|\hat{H}_{\mathrm{eff}}\right| \beta\right\rangle= & \left\langle\alpha\left|\hat{H}_{0}\right| \beta\right\rangle-\frac{1}{2} \sum_{\gamma}\left\langle\alpha\left|\hat{H}_{1}^{\nu m}\right| \gamma\right\rangle\left\langle\gamma\left|\hat{H}_{1}^{\nu m}\right| \alpha\right\rangle \\
& \times\left[\frac{1}{E_{\gamma}-E_{\alpha}}+\frac{1}{E_{\gamma}-E_{\beta}}\right]
\end{aligned}
$$

where $\hat{H}_{0}=\hat{H}_{0}^{\nu}+\hat{H}_{0}^{m}$, given by the sum of the interaction terms (6.4) and (6.5), is diagonal on the states $|\alpha\rangle$, and the single-particle tunneling term 


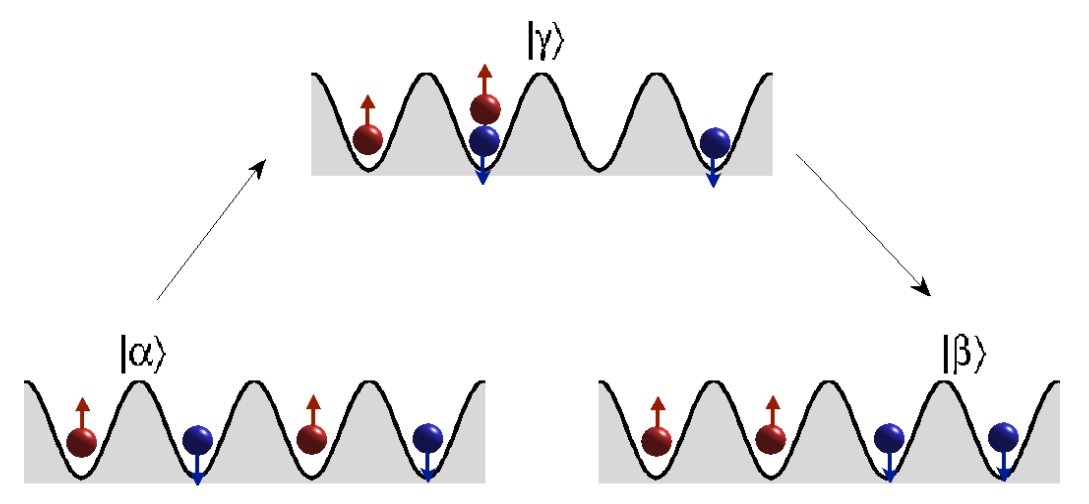

Figure 6.3: Schematic representation of a two-particle hopping between the states $|\alpha\rangle$ and $|\beta\rangle$, belonging to the low-energy subspace at $\nu=1 / 2$. These states are coupled to the virtual excitation $|\gamma\rangle$ through single-particle jumps.

$\hat{H}_{1}^{\nu m}$ of Eq. (6.6) is treated at second order. For a given state $|\alpha\rangle$,

$$
E_{\gamma_{i j}}-E_{\alpha}=U+U_{N N} \Delta m_{\mathrm{NN}}^{i j},
$$

with $\Delta m_{\mathrm{NN}}^{i j}=\sum_{\langle k\rangle_{i}} 2 m_{k}-\sum_{\langle k\rangle_{j}} 2 m_{k}-1$, where $m_{i}$ indicates the population imbalance at site $i$ of Eq. (6.2). For $U \gg U_{N N}$, the denominators $E_{\gamma_{i j}}-E_{\alpha}$ are all of order $U$, which leads to

$$
\begin{aligned}
\hat{H}_{\mathrm{eff}}^{(0)} & =\hat{H}_{0}^{\nu}-\frac{2 J^{2}}{U} \sum_{\langle i j\rangle} \hat{\nu}_{i}\left(\hat{\nu}_{j}+1\right) \\
& +\hat{H}_{0}^{m}-\frac{2 J^{2}}{U} \sum_{\langle i j\rangle}\left[\hat{m}_{i} \hat{m}_{j}+\hat{c}_{i}^{\dagger} \hat{c}_{j}\right],
\end{aligned}
$$

where $\hat{c}_{i}=\hat{a}_{i} \hat{b}_{i}^{\dagger}$ and $\hat{c}_{i}^{\dagger}=\hat{a}_{i}^{\dagger} \hat{b}_{i}$ are the destruction and creation operators of a composite, made of a particle of one species and a hole of the other species, such that

$$
\begin{aligned}
& \hat{c}_{i}\left|\nu, m_{i}\right\rangle=\sqrt{\nu(\nu+1)-m_{i}\left(m_{i}-1\right)}\left|\nu, m_{i}-1\right\rangle \\
& \hat{c}_{i}^{\dagger}\left|\nu, m_{i}\right\rangle=\sqrt{\nu(\nu+1)-m_{i}\left(m_{i}+1\right)}\left|\nu, m_{i}+1\right\rangle,
\end{aligned}
$$

and their commutation relations are given by $\left[\hat{c}_{i}, \hat{c}_{j}^{\dagger}\right]=-2 \hat{m}_{i} \delta_{i j}$, with $\delta_{i j}$ being the Kronecker delta.

For a given $\nu$, the second line of the Hamiltonian (6.14) can be equivalently written in terms of the spin operators at site $i$, given by

$$
\hat{\mathbf{S}}_{i}=\frac{1}{2} \sum_{\mathrm{uu}^{\prime}} \hat{a}_{\mathrm{iu}}^{\dagger} \vec{\sigma}_{\mathrm{uu}^{\prime}} \hat{a}_{\mathrm{iu}^{\prime}}
$$


where $\vec{\sigma}=\left(\sigma_{x}, \sigma_{y}, \sigma_{z}\right)$ are the Pauli matrices, and $u=a, b$ indicates the two species. Thus, the creation and annihilation operators (6.15) become $\hat{c}_{i}^{\dagger}=\hat{S}_{i}^{x}+i \hat{S}_{i}^{y}$ and $\hat{c}_{i}=\hat{S}_{i}^{x}-i \hat{S}_{i}^{y}$ respectively, while the imbalance operator is given by $\hat{m}_{i}=\hat{S}_{i}^{z}$ as already anticipated in Sec. 6.2.1. Therefore, in the spin operators language (6.16), the second line of the Hamiltonian (6.14) looks like a Heisenberg spin Hamiltonian (see e.g. [46]). The chemical potential $\mu_{-}$ plays the role of an external magnetic field along the $z$ axis, and the interplay between $\mu_{-}$, and the nearest-neighbor interactions determines the magnetic ordering of the system, as we will discuss in the next section.

\subsection{Mean-field}

In this section, we provide a mean-field solution to the effective Hamiltonian (6.14) in order to investigate the phases of the system. We identify the different phases through the mean-field composite order parameters $\left\langle\hat{c}_{i}\right\rangle$, as well as both the single-particle ones $\left\langle\hat{a}_{i}\right\rangle$, and $\left\langle\hat{b}_{i}\right\rangle$.

For every subspace at constant filling, we find that the system presents three different kinds of phases. The Mott-insulating phase (MI), with a well defined number of particles at each site of the lattice, and absence of any low-energy transport [1]. The MI is characterized by vanishing $\left\langle\hat{c}_{i}\right\rangle=\left\langle\hat{a}_{i}\right\rangle=$ $\left\langle\hat{b}_{i}\right\rangle=0$, and depending on the value of $\mu_{-}$presents either FM or AM ordering. Instead in the super-counter-fluid phase (SCF), characterized by on-site density fluctuations, the net transport of atoms is still suppressed but a counterflow is present, in which the currents of the two species are equal in absolute value but in opposite directions [78]. In the SCF phase, while the single-particle order parameters still vanish $\left\langle\hat{a}_{i}\right\rangle=\left\langle\hat{b}_{i}\right\rangle=0$, the composite order parameters are non-zero $\left\langle\hat{c}_{i}\right\rangle \neq 0$, indicating the presence of counterflow. In this work we also find evidences for the existence of the novel counterflow supersolid phase (CSS). The CSS is characterized by vanishing single-particle order parameters $\left\langle\hat{a}_{i}\right\rangle=\left\langle\hat{b}_{i}\right\rangle=0$, and non-vanishing composite order parameters $\left\langle\hat{c}_{i}\right\rangle \neq 0$, coexisting with broken translational symmetry, namely, a modulation of both $m_{i}$, and $\left\langle\hat{c}_{i}\right\rangle$ on a scale larger than the one of the lattice spacing, analogously to the supersolid phase.

To determine the insulating phases we perform a perturbative treatment at first order in the composite order parameters $\psi_{i}=\left\langle\hat{c}_{i}\right\rangle$, which allow us to compute the boundaries of the insulating lobes. Furthermore, we solve the time dependent Gutzwiller equations in imaginary time to determine the nature of the SCF-CSS phases outside the lobes. 


\subsubsection{Insulating lobes}

The low-energy subspace is spanned by the classical distribution of atoms in the lattice $|\alpha\rangle$ of Eq. (6.10). Similarly to the two layer system discussed in Sec. 5.2, in the limit of $U \gg U_{\mathrm{NN}}$, asymptotically all classical states $|\alpha\rangle$ become stable with respect to single-particle-hole excitations and develop an insulating lobe at finite $J$. The energy of single particle-hole excitations is of the order of $U$ at $J=0$ and is given by the width of the lobes at finite $J$ (see, e.g., the thin blue lobes in Fig. 6.4).

Instead, the low-lying excitations remain within the subspace and are obtained by adding $(\mathrm{PH})$ or removing (HP) one composite, made of a particle of one species and a hole of the other species, at the $i$-th site of the lattice. This corresponds to flip the direction of a dipole at the site $i$, respectively from down to up (PH) or from up to down (HP). For any given configuration $|\alpha\rangle$, one can calculate their energy costs with the diagonal terms of the effective Hamiltonian (6.14), which are respectively given by

$$
\begin{aligned}
& E_{\mathrm{PH}}^{i}(J)=-2 \mu_{-}+4\left(U_{\mathrm{NN}}-J^{2} / U\right) \sum_{\langle k\rangle_{i}} m_{k}, \\
& E_{\mathrm{HP}}^{i}(J)=2 \mu_{-}-4\left(U_{\mathrm{NN}}-J^{2} / U\right) \sum_{\langle k\rangle_{i}} m_{k} .
\end{aligned}
$$

Notice that in the last expressions, there is no explicit dependence on the chemical potential $\mu_{+}$. This is because by adding or removing one composite, one remains within the subspace at filling factor $\nu$, and therefore the contribution of $\mu_{+}$vanish in the calculation of the excitations (6.17). By using the perturbative mean-field method derived in Sec. 2.2.2, we can calculate the order parameters $\psi_{i}=\left\langle\hat{c}_{i}\right\rangle$ for $|\alpha\rangle$, which satisfy the equations

$$
\psi_{i}=\frac{2 J^{2}}{U}\left[\frac{\nu(\nu+1)-m_{i}\left(m_{i}+1\right)}{E_{\mathrm{PH}}^{i}(J)}+\frac{\nu(\nu+1)-m_{i}\left(m_{i}-1\right)}{E_{\mathrm{HP}}^{i}(J)}\right] \bar{\psi}_{i},
$$

where $\bar{\psi}_{i}=\sum_{\langle j\rangle_{i}} \psi_{j}$. With Eqs. (6.18) one can calculate the mean-field lobes of any distribution of atoms in the lattice $|\alpha\rangle$, providing the elementary excitations (6.17), are positive in some range of the parameters. As we have demonstrated in Sec. 2.2.2 this is a necessary condition for the existence of an insulating lobe and provides its boundaries at $J=0$, given by

$$
2 U_{\mathrm{NN}} \sum_{\langle k\rangle_{i}} m_{k}<\mu_{-}<2 U_{\mathrm{NN}} \sum_{\langle k\rangle_{i}} m_{k} .
$$

To obtain the last inequalities, one has to be careful and flip the direction of a dipole only where it is possible. For example, suppose the site $i$ is occupied only by one particle of the species $a$, i.e $m_{i}=1 / 2$, then for this site 
the conditions $(6.19)$ reduce to $\mu_{-}>2 U_{\mathrm{NN}} \sum_{\langle k\rangle_{i}} m_{k}$, since the only possible excitation at this site corresponds to remove a composite. As usual, to find the boundaries of the insulating lobes we use the procedure explained in Sec. 2.2.2. For each site of the lattice one has such a condition (6.19), and an Eq. (6.18). The latter is a set of coupled equations, which can be written in the matrix form $\mathcal{M}\left(\mu_{-}, U, U_{\mathrm{NN}}, J\right) \cdot \vec{\psi}=0$, with $\vec{\psi} \equiv\left(\cdots \psi_{i} \cdots\right)$ being the vector of the order parameters at each site of the lattice. For every $\mu_{-}$, a non-trivial solution is provided by the smallest $J$ for which $\operatorname{det}\left[\mathcal{M}\left(\mu_{-}, U, U_{\mathrm{NN}}, J\right)\right]=0$, that is the insulating lobe of the $|\alpha\rangle$ configuration in the $J$ vs. $\mu_{-}$plane. In Fig. 6.5 we plot the ground state insulating lobes calculated in this way for $\nu=1 / 2$ (left) and $\nu=1$ (right). For all filling factors $\nu$, we find an AM ground state $(\nu, M=0)$, which presents a spatial distribution of alternating sites occupied by particles of species $a$ and $b$ resembling a checkerboard structure. Instead, by increasing the absolute value of $\mu_{-}$we find a FM ground state $(\nu, M= \pm \nu)$, in which only particles of one type are present. It is worth noticing that the insulating lobes calculated in this way, do not contain any dependence on $\mu_{+}$, which does not enter into Eqs. (6.18) as previously discussed. Therefore, to obtain the complete 3D phase diagram, one has to compare the energies of the ground state configurations at different $\nu$. Using the effective Hamiltonian (6.14), for any value of $J, \mu_{-}$, and $\mu_{+}$, we calculate the energies of the ground state configurations for different $\nu$, and select the state with the smaller energy. In this way we have obtained for example the phase diagram at $J=0$ of Fig. 6.2.

\subsubsection{Counterflow superfluid-supersolid}

In the low-energy subspace at constant $\nu$, the Gutzwiller Ansatz on the wave function of the system reads

$$
|\Phi\rangle=\prod_{i} \sum_{\mathrm{m}=-\nu}^{\nu} f_{\nu, \mathrm{m}}^{(i)}|\nu, m\rangle_{i}
$$

where we allow the Gutzwiller amplitudes to dependent on time $f_{\nu, \mathrm{m}}^{(i)}(t)$. As in Sec. 2.2.1, we obtain the equations of motion for the amplitudes by minimizing the action of the system, given by $S=\int \mathrm{d} t \mathcal{L}$, with respect to the variational parameters $f_{\nu, \mathrm{m}}^{(i)}(t)$ and their complex conjugates $f_{\nu, \mathrm{m}}^{*(i)}(t)$, where

$$
\mathcal{L}=\frac{\langle\Phi \mid \dot{\Phi}\rangle-\langle\dot{\Phi} \mid \Phi\rangle}{2 i}-\left\langle\Phi\left|\hat{H}_{\mathrm{eff}}^{(0)}\right| \Phi\right\rangle,
$$




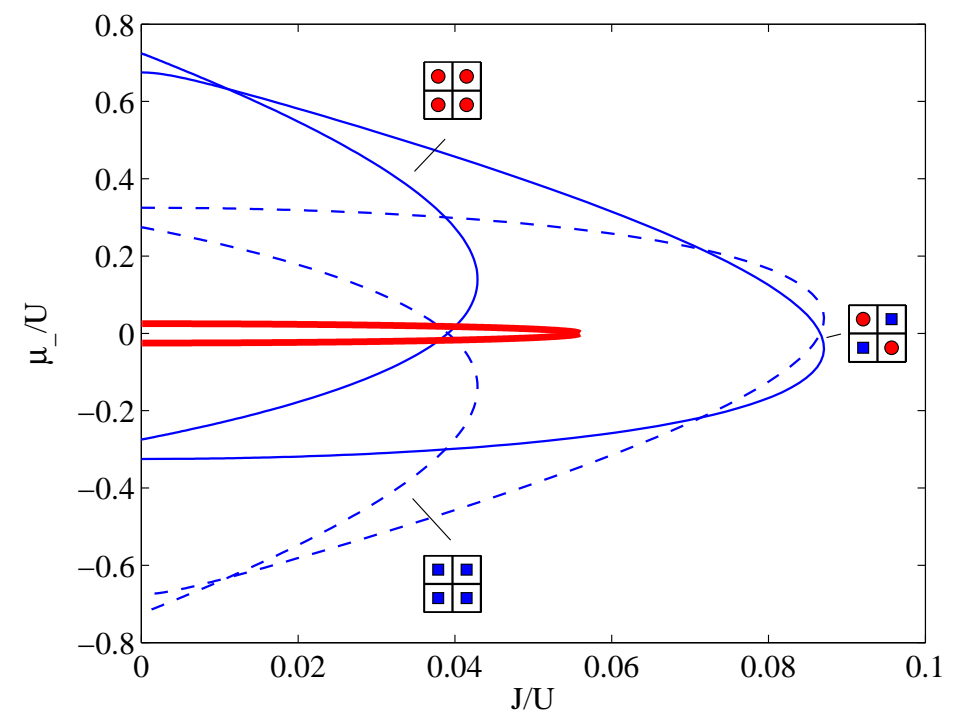

Figure 6.4: Insulating lobes at $\nu=1 / 2$ filling, with $U_{\mathrm{NN}}=U / 160$. The thick line is the anti-ferromagnetic insulating state calculated with the effective Hamiltonian $\hat{H}_{\text {eff }}^{(0)}$. The thin blue lines (dashed lines) represent insulating lobes calculated with respect to single-particle-hole excitations of the species $a$ (species $b$ ), sketched as a plain dot (square), and are computed for $\mu_{+}=$ $0.65 U$.

is the Lagrangian of the system in the quantum state $|\Phi\rangle[60]$. Therefore by equating to zero the variation of the action with respect to $f_{\nu, \mathrm{m}}^{*(i)}$, leads to the equations

$$
\begin{aligned}
i \hbar \frac{\mathrm{d}}{\mathrm{d} t} f_{\nu, \mathrm{m}}^{(i)} & =\left[-2 \mu_{-}+4\left(U_{N N}-J^{2} / U\right) \sum_{\langle j\rangle_{i}}\left\langle\hat{m}_{j}\right\rangle\right] m f_{\nu, \mathrm{m}}^{(i)} \\
& -\frac{2 J^{2}}{U}\left[\bar{\psi}_{i} \sqrt{\nu(\nu+1)-m(m-1)} f_{\nu, \mathrm{m}-1}^{(i)}\right. \\
& \left.+\bar{\psi}_{i}^{*} \sqrt{\nu(\nu+1)-m(m+1)} f_{\nu, \mathrm{m}+1}^{(i)}\right]
\end{aligned}
$$

where $\left\langle\hat{m}_{i}\right\rangle=\sum_{\mathrm{m}=-\nu}^{\nu} m\left|f_{\nu, \mathrm{m}}^{(i)}\right|^{2}$, the fields $\bar{\psi}_{i}=\sum_{\langle j\rangle_{i}} \psi_{j}$ and $\sum_{\langle j\rangle_{i}}\left\langle\hat{m}_{j}\right\rangle$, have to be calculated in a self consistent way as explained in Sec. 2.2.1, and the order parameter is given by

$$
\psi_{i}=\left\langle\Phi\left|\hat{c}_{i}\right| \Phi\right\rangle=\sum_{\mathrm{m}=-\nu}^{\nu} \sqrt{\nu(\nu+1)-m(m+1)} f_{\nu, \mathrm{m}}^{*(i)} f_{\nu, \mathrm{m}+1}^{(i)} .
$$


We solve Eqs. (6.22) in imaginary time $\tau=i t$, which due to dissipation is supposed to converge to the ground state. In Fig. 6.5 we show the ground state phase diagram of the system for $\nu=1 / 2$ (left) and $\nu=1$ (right), computed in this way for $U_{\mathrm{NN}}=U / 160$. In the region outside the insulating AM lobes and enclosed between the FM states, depending on the values of $J$ and $\mu_{-}$we find either SCF or CSS. The CSS phase is characterized by vanishing single-particle order parameters $\left\langle\hat{a}_{i}\right\rangle=\left\langle\hat{b}_{i}\right\rangle=0$, coexisting with a spatial modulation of the composite order parameters $\left\langle\hat{c}_{i}\right\rangle \neq 0$, indicating the presence of counterflow. The shaded areas in Fig. 6.5 indicate where $\left\langle\hat{c}_{i}\right\rangle \neq 0$, and present a spatial modulation. These have to be compared with the region where the single-particle order parameters are zero, in order to determine the limits of validity of the CSS phase. For $\nu=1 / 2$, the thin blue lines (dashed lines) in Fig. 6.4 represent the insulating lobes calculated with respect to single-particle-hole excitations of the species $a$ (species $b$ ). The two lobes extending up to $J \sim 0.09 U$, correspond to the AM insulating ordering, they delimit the extension of the CSS phase, and they give an estimate of the limits of validity of $\hat{H}_{\text {eff }}^{(0)}$, beyond which the subspace of constant $\nu$ looses its meaning.

We have already mentioned, that the boundaries of the lobes calculated with the effective Hamiltonian do not show any dependence on the chemical potential $\mu_{+}$, which does not give any contribution in the expression of the low-lying excitations (6.17). This is not true in the case of the single-particle-hole insulating lobes, since adding or removing a single particle results in a change of both $\mu_{+}$, and $\mu_{-}$. This makes the process of estimating the limits of validity of $\hat{H}_{\mathrm{eff}}^{(0)}$ more complicated and leads to a $3 \mathrm{D}$ phase diagram in the $J, \mu_{+}$, and $\mu_{-}$variables highly non-trivial, a systematic study of which is still on going. 

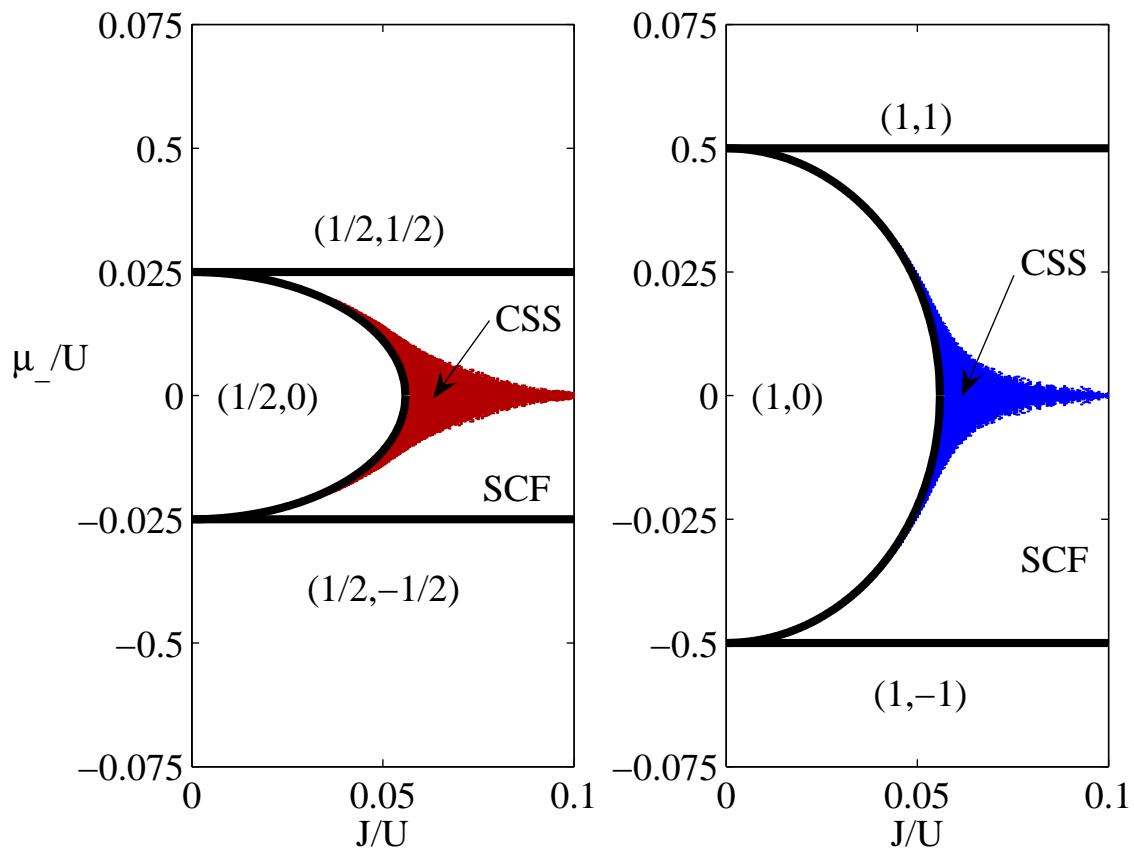

Figure 6.5: Ground state of a $2 \times 2$ square lattice satisfying periodic boundary conditions, for $\nu=1 / 2$ (left) and $\nu=1$ (right), and $U_{\mathrm{NN}}=U / 160$. The text in parenthesis $(\nu, M)$ indicate the filling factor $\nu$, and the average magnetization $M$, respectively. 


\section{Chapter 7}

\section{Conclusions}

Summarizing, we have studied the phase diagram of a bilayer system of $2 \mathrm{D}$ dipolar lattice gases, in the limit of close layers, and demonstrated the existence of a novel PSS phase, namely a supersolid phase of pairs. The existence of the PSS phase has been previously discussed for anisotropic $t$ - $J$ models [80], but no evidence of it has been found. However, the Hamiltonian we discuss in the present work differs from the anisotropic $t-J$ spin Hamiltonian in three crucial respects, all of which should favor the existence of the PSS phase: (i) it deals with soft-core Bosons (vs. hard-core); (ii) it considers onsite inter-species attraction (vs. nearest-neighbor inter-species attraction); (iii) it includes nearest-neighbor intra-species repulsion. For these reasons, we believe that the existence of the PSS phase will be confirmed by exact calculations, also beyond the limits of validity of our effective mean-field approach.

Currently we are studying a 2D lattice of dipolar Bosons, where the dipoles are free to point in both directions perpendicularly to the plane, which results in a nearest neighbor repulsive (attractive) interaction for aligned (anti-aligned) dipoles. This system is interesting from a theoretical point of view, because it shows many similarities with the bilayer system previously described, as well as from an experimental one in view of its possible realizations with $\mathrm{OH}$ molecules. In a mean-field approach, we have found regions of parameters where the ground state of the system is ferromagnetic or antiferromagnetic, and found evidences for the existence of a novel Counterflow Super Solid (CSS) quantum phase. Nevertheless a systematic study of the complete phase diagram is still on going. 


\section{Part IV}

\section{Quantum Monte Carlo}




\section{Introduction}

Any physical system consisting of $N$ non-relativistic particles can be in principle described by the many-body Schrödinger equation. In three dimensions (3D), the number of degrees of freedom in the Schrödinger equation becomes 3 times $N$, which for typical physical systems such as electrons in conducting materials or BEC that have a large number of constituents $N$, it becomes difficult to solve exactly, even for parallel computing in a reasonable amount of time. Monte Carlo methods overcome this problem, they allow for a description of the many-body system relying on repeated random sampling, at the cost of statistical uncertainty which can be reduced with more simulation time. The typical basic steps of any Monte Carlo algorithm can be summarized as follows

1. Define a domain of possible inputs, the Configuration Space.

2. Generate inputs randomly from the domain with a certain probability distribution that depends on the specific problem. This is called the updating procedure.

3. Perform a computation based on the randomly generated input.

4. Add the result of the computation to the final result.

There is a large class of Quantum Monte Carlo algorithm that can simulate quantum many-body systems, like for example the Variational Monte Carlo [34, 35], the Diffusion Monte Carlo [28, 27, 29], the Path Integral Monte Carlo [33, 81, 82], auxiliary field Monte Carlo [36, 37], etc. Most methods aim at computing the ground-state wavefunction of the system, with the exception of Path Integral Monte Carlo, and finite-temperature auxiliary field Monte Carlo, which calculate the density matrix. The result presented in this thesis work are based on the Path Integral Monte Carlo (PIMC) and the Worm Algorithm (WA), which was originally developed by Prokof'ev, Svistunov and Tupitsyn [81, 82].

The work is organized as follows: first in Sec. 8.1 we introduce the reader with the general ideas of the PIMC and then we study the concrete case of a 
2D dipolar Hamiltonian in Sec 8.1.1. We then discuss the characteristics of the WA, and in particular its unique updating procedure in Sec. 8.2. Finally we present and discuss our results [86] in Chapter 9.

Our results are based on the publication:

- B. Capogrosso-Sansone, C. Trefzger, M. Lewenstein, P. Zoller, and G. Pupillo, Quantum Phases of Cold Polar Molecules in 2D Optical Lattices. arXiv:0906.2009. Accepted for Physical Review Letters publication. 


\section{Chapter 8}

\section{Path Integral Monte Carlo and the Worm algorithm}

\subsection{Path Integral Monte Carlo}

Consider a system described by the Hamiltonian $\hat{H}=\hat{H}_{0}+\hat{H}_{1}$, where $\hat{H}_{0}$ is diagonal in the basis of eigenstates $\{|\alpha\rangle\}$ satisfying the eigenvalue equation

$$
\hat{H}_{0}|\alpha\rangle=E_{\alpha}|\alpha\rangle,
$$

and $\hat{H}_{1}$ is non-diagonal. The thermodynamic properties of the system at equilibrium, can be derived from the partition function which is given by the trace of the density matrix operator $Z=\operatorname{Tr}\left[e^{-\beta \hat{H}}\right]$, where $\beta=1 / K_{B} T$ is the inverse temperature and $K_{B}$ the Boltzmann constant. In the interaction picture [56] one may write

$$
Z=\operatorname{Tr}\left[e^{-\beta\left(\hat{H}_{0}+\hat{H}_{1}\right)}\right]=\operatorname{Tr}\left[e^{-\beta \hat{H}_{0}} \hat{\mathcal{T}}_{\tau} e^{-\int_{0}^{\beta} \mathrm{d} \tau \hat{H}_{1}(\tau)}\right],
$$

where $\hat{\mathcal{T}}_{\tau}$ is the time-ordering operator, $\hat{H}_{1}(\tau)=e^{\tau \hat{H}_{0}} \hat{H}_{1} e^{-\tau \hat{H}_{0}}$ is the nondiagonal part of the Hamiltonian expressed in the interaction picture, and the variable $\tau$ is usually called the imaginary time $e^{1}$. One can write the partition function using the Feynman path integral formulation, and by Taylor

\footnotetext{
${ }^{1}$ This is because by replacing $\tau=i t$, with $t$ being the real time, the operator $e^{-\tau \hat{H}}$ becomes the usual time-evolution operator in quantum mechanics.
} 
expanding the second exponent in the right-hand-side of Eq. (8.2) one gets

$$
\begin{aligned}
Z & =\sum_{\alpha} e^{-\beta E_{\alpha}}\langle\alpha| \hat{\mathbb{1}}-\int_{0}^{\beta} \mathrm{d} \tau \hat{H}_{1}(\tau)+ \\
& +\sum_{m=2}^{\infty}(-1)^{m} \int_{0}^{\beta} \mathrm{d} \tau_{\mathrm{m}} . . \int_{0}^{\tau_{2}} \mathrm{~d} \tau_{1} \hat{H}_{1}\left(\tau_{\mathrm{m}}\right) . . \hat{H}_{1}\left(\tau_{1}\right)|\alpha\rangle
\end{aligned}
$$

where the integrals are ordered in time and the sum over the states $|\alpha\rangle$ comes from the trace. Now we explicitly make use of the completeness property of the $\{|\alpha\rangle\}$ base, and insert $m-1$ identity operators $\hat{\mathbb{1}}=\sum_{\alpha}|\alpha\rangle\langle\alpha|$ between the products of $\hat{H}_{1}\left(\tau_{\mathrm{m}}\right)$ operators, therefore we can write

$$
\left\langle\alpha\left|\hat{H}_{1}\left(\tau_{m}\right) . . \hat{H}_{1}\left(\tau_{1}\right)\right| \alpha\right\rangle=\sum_{\alpha_{1}, . ., \alpha_{\mathrm{m}-1}} H_{1}^{\alpha \alpha_{\mathrm{m}-1}}\left(\tau_{\mathrm{m}}\right) . . H_{1}^{\alpha_{2} \alpha_{1}}\left(\tau_{2}\right) H_{1}^{\alpha_{1} \alpha}\left(\tau_{1}\right)
$$

where the matrix elements

$$
H_{1}^{\alpha^{\prime} \alpha}(\tau)=e^{\tau E_{\alpha^{\prime}}} H_{1}^{\alpha^{\prime} \alpha} e^{-\tau E_{\alpha}}=\left\langle\alpha^{\prime}\left|\hat{H}_{1}\right| \alpha\right\rangle e^{-\tau\left(E_{\alpha}-E_{\alpha^{\prime}}\right)},
$$

contain both diagonal $\left(E_{\alpha}\right)$ and off-diagonal $\left(H_{1}^{\alpha^{\prime} \alpha}\right)$ matrix elements. We now insert the last equation into expression (8.3) and get the final expression for the partition function

$$
\begin{aligned}
Z & =\sum_{\alpha} e^{-\beta E_{\alpha}}\left\{1-\int_{0}^{\beta} \mathrm{d} \tau H_{1}^{\alpha \alpha}(\tau)+\right. \\
& \left.+\sum_{\mathrm{m}=2}^{\infty}(-1)^{m} \int_{0}^{\beta} \mathrm{d} \tau_{\mathrm{m}} . . \int_{0}^{\tau_{2}} \mathrm{~d} \tau_{1} \sum_{\alpha_{1}, . ., \alpha_{\mathrm{m}-1}} H_{1}^{\alpha \alpha_{\mathrm{m}-1}}\left(\tau_{\mathrm{m}}\right) . . H_{1}^{\alpha_{1} \alpha}\left(\tau_{1}\right)\right\}
\end{aligned}
$$

which contains only matrix elements of the operators $\hat{H}_{0}$ and $\hat{H}_{1}$. Therefore, by using this formalism of path integrals, the calculation of the partition function reduces to a classical problem since only scalars enter into Eq. (8.6), but we have payed the price of the extra dimension $\tau$. In other words, the original $d$-dimensional quantum system is equivalent to a $(d+1)$-dimensional classical system.

It is worth noticing that since the partition function is a trace, periodic boundary conditions in the imaginary time $\tau$ must apply. This is easily understood by looking at the $m$-th order term of $Z$, which contains the product of $m$ matrix elements $H_{1}^{\alpha \alpha_{\mathrm{m}-1}}\left(\tau_{\mathrm{m}}\right) \ldots H_{1}^{\alpha_{1} \alpha}\left(\tau_{1}\right)$ that are ordered in time from the first at $\tau_{1}$, to the last at $\tau_{m}$. Therefore, for any given $\alpha$ in the trace, the first matrix element brings $\alpha$ to some $\alpha_{1}$ in the time $\tau_{1} \geq 0$, 
while the last matrix element brings $\alpha_{\mathrm{m}-1}$ back to $\alpha$ in the time $\tau_{\mathrm{m}} \leq \beta$. All the possible configurations which are periodic in imaginary time and that enter into the expression for the partition function Eq. (8.6), define the configuration space spanned by a PIMC algorithm.

\subsubsection{Path Integral Monte Carlo and the 2D extended Bose-Hubbard model}

We now consider a 2D system of $L \times L$ sites filled with polarized dipolar Bosons, we assume spatial periodic boundary conditions and the dipoles to be polarized perpendicularly to the $2 \mathrm{D}$ plane as explained in Chapter 3 . The system is therefore described by the extended Bose-Hubbard Hamiltonian (3.1), which, to be consistent with the notations in our publication [86], we rewrite in this form

$$
\hat{H}=-J \sum_{\langle i j\rangle}\left[\hat{b}_{i}^{\dagger} \hat{b}_{j}+\hat{b}_{i} \hat{b}_{j}^{\dagger}\right]+\sum_{i}\left[\frac{U}{2} \hat{n}_{i}\left(\hat{n}_{i}-1\right)-\mu_{i} \hat{n}_{i}\right]+V \sum_{i<j} \frac{\hat{n}_{i} \hat{n}_{j}}{r_{i j}^{3}},
$$

where $\hat{b}_{i}^{\dagger}\left(\hat{b}_{i}\right)$ is the boson creation (annihilation) operator at site $i, \hat{n}_{i}=\hat{b}_{i}^{\dagger} \hat{b}_{i}$ is the number operator, $V=D / a^{3}>0$ is the dipole-dipole interaction strength $D$ divided by the lattice spacing $a, r_{i j}=|i-j|$ is the distance between two sites of the lattice, and $\mu_{i}=\mu-\Omega i^{2}$ contains the chemical potential $\mu$ which fixes the number of particles and the curvature $\Omega$ of an external harmonic confinement.

We choose to work in the basis of the interaction term of the Hamiltonian (8.7), i.e. Fock states $|\alpha\rangle=\prod_{i}^{L^{2}}\left|n_{i}\right\rangle_{i}$ of localized particles in the $L \times L$ square lattice, where $n_{i}$ is the occupation number at site $i$. Therefore in this basis, the diagonal matrix elements entering Eq. (8.5) take the form

$$
E_{\alpha}=\frac{U}{2} \sum_{i} n_{i}\left(n_{i}-1\right)-\sum_{i} \mu_{i} n_{i}+V \sum_{i<j} \frac{n_{i} n_{j}}{r_{i j}^{3}}
$$

while the off-diagonal ones, are given by the expression

$$
-H_{1}^{\alpha^{\prime} \alpha}=2 J\left\langle\alpha^{\prime}\left|\hat{b}_{i}^{\dagger} \hat{b}_{j}\right| \alpha\right\rangle=2 J \sqrt{\left(n_{i}^{\alpha}+1\right) n_{j}^{\alpha}}
$$

and they connect states $\left|\alpha^{\prime}\right\rangle$ and $|\alpha\rangle$ that differ only in the occupation number of the two nearest neighboring sites $i$ and $j$, namely $\left|\alpha^{\prime}\right\rangle \equiv \frac{\hat{b}_{i}^{\dagger} \hat{b}_{j}}{\sqrt{\left(n_{i}^{\alpha}+1\right) n_{j}^{\alpha}}}|\alpha\rangle$ with $n_{i}^{\alpha}$ being the number of particles at the $i$-th site of the state $|\alpha\rangle$. It is important to notice that we do not use any cutoff in the range of the dipole-dipole interaction entering Eq. (8.8). 
To write the partition function for the 2D extended Bose-Hubbard model, we notice that the first order term vanishes since the matrix elements (8.9) are off-diagonal, i.e. $H_{1}^{\alpha \alpha}=0$, and due to the geometry of the system $(2 \mathrm{D}$ square lattice) it is not difficult to see that all the terms with an odd value of $m$ also vanish. Therefore by rearranging the exponentials and renaming $\alpha \equiv \alpha_{0}$, we get to the expression

$$
\begin{aligned}
Z_{\mathrm{eBH}} & =\sum_{\alpha_{0}} e^{-\beta E_{\alpha_{0}}}+\sum_{\mathrm{m}=2}^{\infty}(-2 J)^{m} A_{\mathrm{m}} \times \\
& \times \int_{0}^{\beta} \mathrm{d} \tau_{\mathrm{m}} \cdot . \int_{0}^{\tau_{2}} \mathrm{~d} \tau_{1} \sum_{\alpha_{0}, \alpha_{1}, . ., \alpha_{\mathrm{m}-1}} \exp \left\{-\beta E_{\alpha_{0}}-\sum_{p=0}^{m-1} E_{\alpha_{p}}\left(\tau_{p+1}-\tau_{p}\right)\right\},
\end{aligned}
$$

where $A_{\mathrm{m}}$ is a product of $m$ square root factors coming from Eq. (8.9) and we have introduced $\tau_{0}=\tau_{m}$ to compact the notation. We can compact further the notation by noticing that for $m=0$ two things happens: (i) the sum in the exponent of Eq. (8.10) does not make any sense, since it is the term of order $m \geq 2$ in the Taylor expansion, therefore we define it to be zero, and (ii) in the sum over the $\alpha$-s only $\alpha_{0}$ survives. Keeping these two considerations in mind and defining $A_{\mathrm{m}=0}=1$, we then write the partition function in the compact form

$$
\begin{aligned}
Z_{\mathrm{eBH}} & =\sum_{\mathrm{m}=0}^{\infty} \sum_{\alpha_{0}, \alpha_{1}, . ., \alpha_{\mathrm{m}-1}}(-2 J)^{m} A_{\mathrm{m}} \times \\
& \times \int_{0}^{\beta} \mathrm{d} \tau_{\mathrm{m}} \cdot \cdot \int_{0}^{\tau_{2}} \mathrm{~d} \tau_{1} \exp \left\{-\beta E_{\alpha_{0}}-\sum_{p=0}^{m-1} E_{\alpha_{p}}\left(\tau_{p+1}-\tau_{p}\right)\right\} .
\end{aligned}
$$

From the last expression one can formally write

$$
Z_{\mathrm{eBH}}=\sum_{\nu} W_{\nu}
$$

with $W_{\nu}$ being the weight of each configuration $\nu \equiv\left[m, \alpha_{0}(\tau), \alpha_{1}(\tau), . ., \alpha_{\mathrm{m}-1}(\tau)\right]$, where not only the $\alpha$-s define $\nu$ but also their distribution in the imaginary time. This is better understood from Fig. 8.1, where we sketch one of such configurations for the 2D lattice.

The imaginary time, $\tau$, is on the horizontal axis, while on the vertical axis there are all the sites of the lattice. Each line is called a worldline and it represents a number of particles proportional to the width of the line: the 


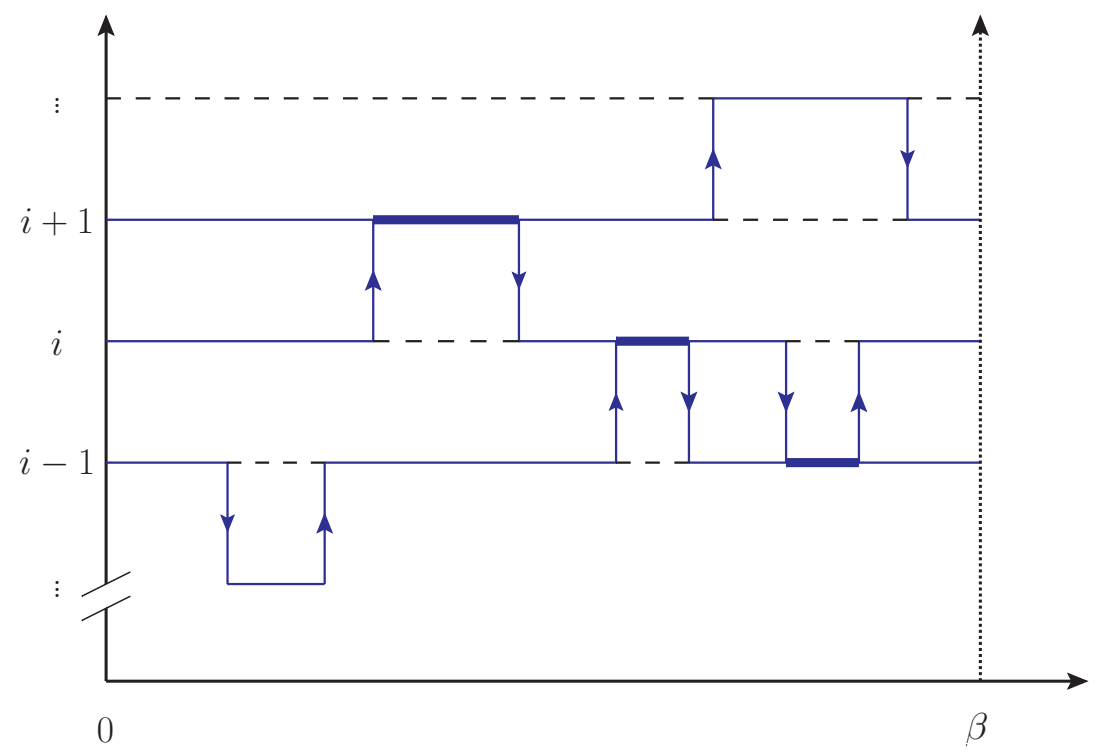

Figure 8.1: Schematic representation of one configuration which enters the calculation of $Z_{\mathrm{eBH}}$. Each worldline represent a number of particles proportional to the width of the line, where the dashed black line is for $n$ particles while the solid and bold blue lines have occupation numbers equal to $n+1$ and $n+2$. Worldlines have to fulfill periodic boundary conditions in the imaginary time $\tau$, and the vertical arrows in correspondence of changes in the occupation numbers are called kinks.

dashed black line is for $n$ particles while the solid and bold blue lines have occupation numbers equal to $n+1$ and $n+2$ respectively. Because the partition function is a trace, worldlines have to close on themselves and since we have also assumed spatial periodic boundary conditions one can imagine the configuration of Fig. 8.1 to be wrapped on a torus. We call the phase space of all possible configurations the closed path configuration space $(\mathrm{CP})$, which is spanned by the PIMC algorithm.

If we cut one configuration at a certain instant in imaginary time, we get the quantum system in a particular state, and the points in imaginary time where the system changes state are called kinks, which in Fig. 8.1 are represented by vertical arrows. A configuration with a number of kinks equal to $m$, contributes to the $m$-th order term of the partition function Eq. (8.11), and it is straightforward to see that there exist an infinite number of different configurations with the same number of kinks, the difference being the time at which the kinks take place and/or the different states they connect. The updating procedure of a PIMC algorithm therefore consists of changing the number of kinks and/or their position in imaginary time. We will discuss the 
updating procedure specifically for the Worm algorithm in the next section.

\subsection{The Worm algorithm}

The Worm Algorithm, which was originally developed by Prokof'ev, Svistunov and Tupitsyn [81, 82], works in an enlarged configuration space, in which one allows one disconnected worldline, the worm, drawn as a red line in Fig. 8.2. This is equivalent to work in the Grand-Canonical ensemble, as we shall discuss in Sec. 8.2.1, and the disconnected worldline allow to efficiently collect statistics for calculating the Matsubara Green function, defined as

$$
G(j, \tau)=\left\langle\hat{\mathcal{T}}_{\tau} \hat{b}_{\mathrm{i}+\mathrm{j}}\left(\tau_{0}+\tau\right) \hat{b}_{\mathrm{i}}^{\dagger}\left(\tau_{0}\right)\right\rangle,
$$

where $\hat{\mathcal{T}}_{\tau}$ is the time-ordering operator, $\tau_{0}$ and $\tau$ are two points in imaginary time, $i$ and $j$ are two sites of the lattice, and the symbol $\langle$.$\rangle stands for the$ statistical average of the expectation value of an operator. Due to space and imaginary time translational invariance of the system, the Green function Eq. (8.13) does not depend on $i$ and $\tau_{0}$. The configuration space of the Matsubara Green function is called the $C P_{\mathrm{g}}$ space, and it is easy to see that the only difference between configurations contributing to the partition function $Z_{\mathrm{eBH}}$ and those contributing to the Green function $G$ is that, for the latter, one of the worldlines starts at $\left(i, \tau_{0}\right)$ and ends at $\left(i+j, \tau_{0}+\tau\right)$, i.e. the worldline is disconnected.

\subsubsection{Updating procedures}

Let us now discuss the updating procedure of the Worm Algorithm, that is when the system is in a certain configuration $\nu$ and the algorithm has to generate randomly a new configuration $\nu^{\prime}$ to collect statistics for evaluating the observables of interest. Notice that in order to ensure ergodicity, and therefore the reliability of the statistics, the updating process must be fully random such as to cover enough of the phase space of the system in a reasonable amount of time.

Apart from the creation of a worm, which is done in the $C P$ space, all other updates are done in the $C P_{\mathrm{g}}$ space through the two ends of the worm. One can picture the updating scheme as sequence of 'drawing' and 'erasing' procedures, happening at the end points of the worm. Given the configuration $\nu$, the algorithm selects randomly an interval of the configuration in imaginary time, which we call a time-interval. Below we list and describe the four types of updates the Worm Algorithm goes through. 


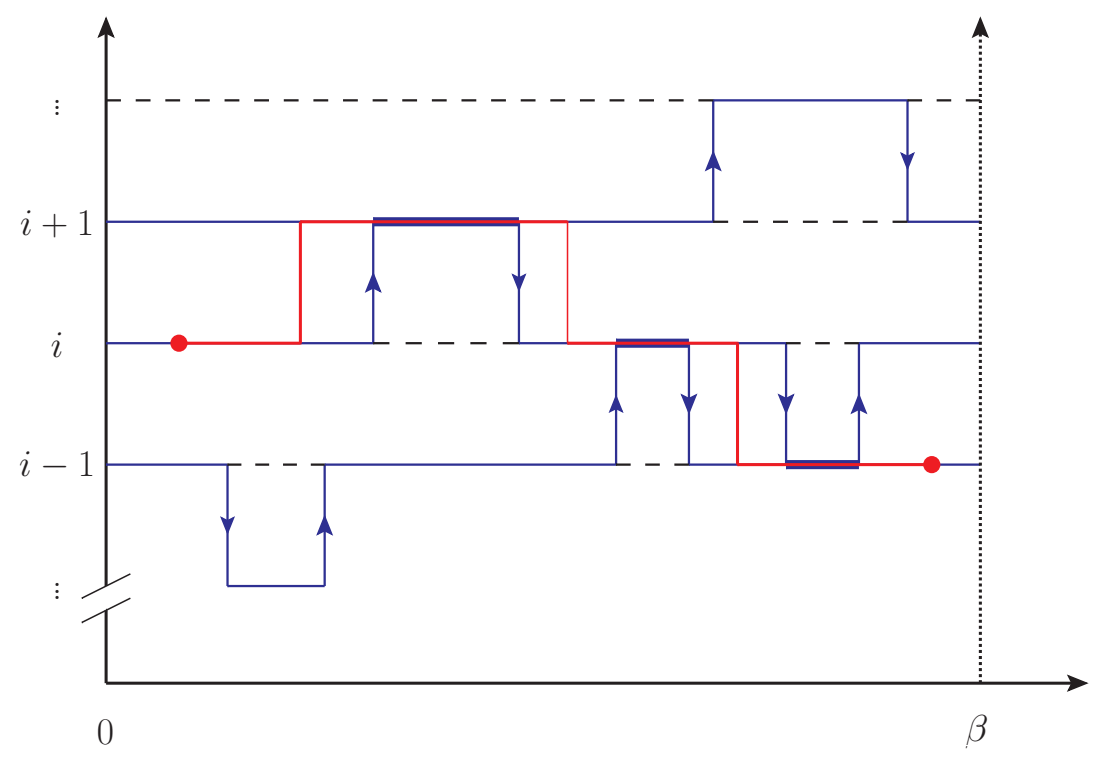

Figure 8.2: Configuration of the $C P_{\mathrm{g}}$ space, the red disconnected line represents the worm.

\section{Creation of a worm}

Creating a worm is the only update performed in the $C P$ space, therefore the starting point is a configuration $\nu$ belonging to $C P$. One of the worldlines of $\nu$ is randomly selected, and on that worldline a time interval is also selected at random, e.g. the interval $n_{1}$ in Fig. 8.3 delimited by $\tau_{\min }$ and $\tau_{\max }$ indicated by the crosses. Then the program takes randomly two points on the segment $n_{1}$, say $\tau_{1}$ and $\tau_{2}$, which will be the worm extremities indicated by plain dots in Fig. 8.3, and the condition $\tau_{\min }<\tau_{1}<\tau_{2}<\tau_{\max }$ has to be satisfied. With equal probability one suggests to draw a piece of worldline or delete a piece from an existing worldline, with the constraints that the resulting configuration belongs to the Hilbert space, i.e. it is not possible to erase from an empty interval or to draw on an interval which has reached the maximum occupation number allowed, if any. The worm is therefore created and all other updates will take place through its two extremities.

\section{Deletion of a worm}

In analogy, the opposite updating process which is the deletion of a worm, can only take place in the $C P_{\mathrm{g}}$ space and only if the two extremities of the worm belong to the same worldline so as to assure that after having deleted a worm the system is in a $C P$ state. 


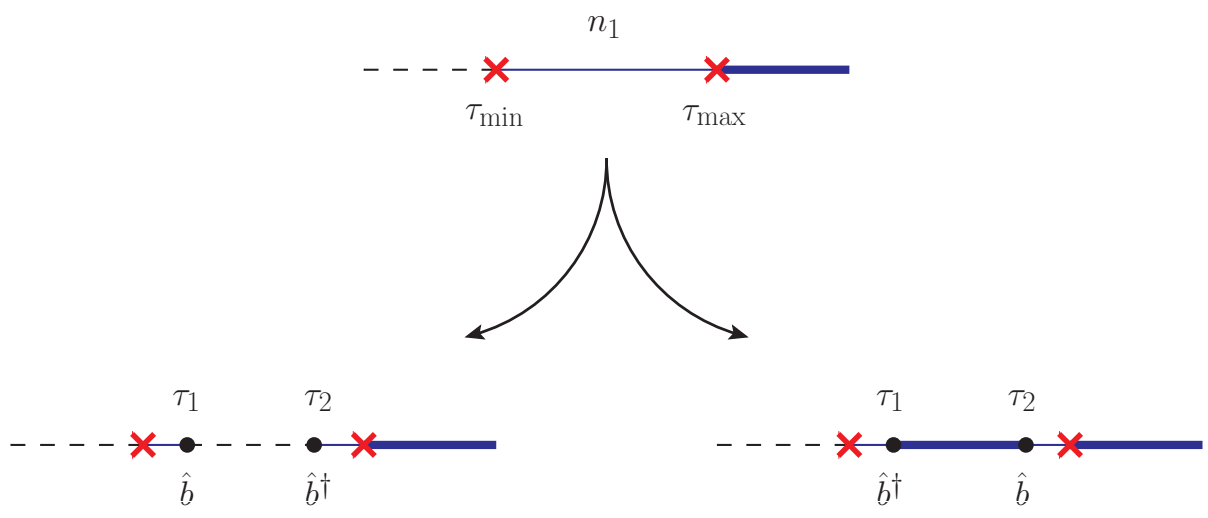

Figure 8.3: Creation of a worm. From a given configuration, one worldline is randomly chosen (top), in which a time interval delimited by $\tau_{\min }$ and $\tau_{\max }$ is randomly selected. Then within the interval, two points $\tau_{1}$ and $\tau_{2}$ are also chosen randomly and will be the two extremities of the worm. With equal probability one can choose to delete a piece of worldline (bottom left) or draw a piece of worldline (bottom right) and the worm is therefore created.

\section{Time shift}

This is the simplest of the updates and it consists of moving one of the extremities of the worm in a random point of the imaginary time, such as to lengthen or shorten the size of the worm. The program selects randomly a time interval, delimited by $\tau_{\min }$ and $\tau_{\max }$, and in this interval only one point is randomly chosen, which is the point where either the head or the tail of the worm is moved to.

\section{Space shift}

This update changes the number of kinks and it consists of creating or delating a kink to the left (space shift left) or to the right (space shift right) of the operator $\hat{b}$ (or $\hat{b}^{\dagger}$ ). Fig. 8.4(a) shows the creation of a kink backward in imaginary time, i.e. the space shift left. Two neighboring worldlines are selected at random as for example $i$ and $j$ of Fig. 8.4(a), then based on the current position of the operator $\hat{b}$ the program chooses randomly a time interval delimited by $\tau_{\min }$ and $\tau_{\max }$. Within this interval the program selects randomly a point where to create or delete a kink, as for example shown in Fig. 8.4(a) for the creation process, with the requirement that the created or deleted kink does not interfere with any other kinks.

The last update, the space shift right shown in Fig. 8.4(b), is equivalent to the left one with the only difference that the kink is inserted or deleted to 

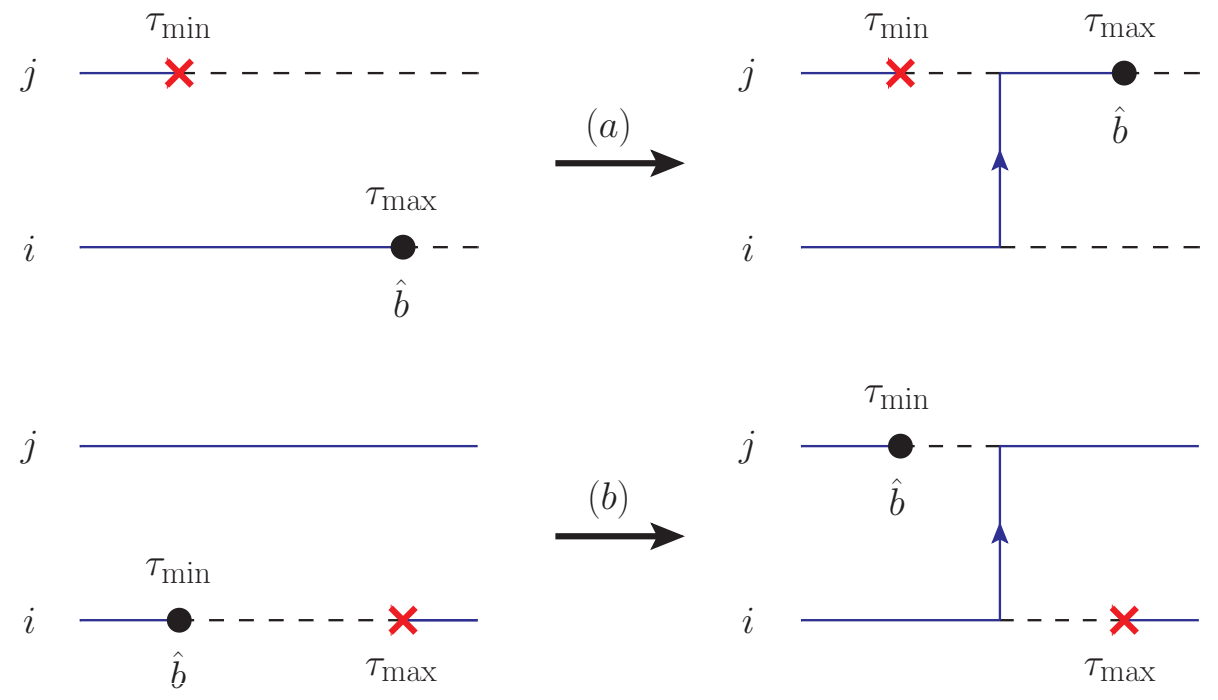

Figure 8.4: Sketch of space shift updates that create or delete kinks. In the space shift left (a), one kink is created backward in the imaginary time and therefore to the left of the operator $\hat{b}$, while in the space shift right the kink is inserted to the right of $\hat{b}$, i.e. forward in the imaginary time.

the right side of $\hat{b}$ operator, i.e. forward in imaginary time.

These are all the updates performed by the WA. From these, it is straightforward to see that the WA works in the Grand Canonical ensemble where the chemical potential becomes an input parameter which fixes the average particle number. For example, suppose the algorithm starts with an initial configuration $\nu$ of zero particles in the system, i.e. the analogous representation of Fig. 8.1 would be a bunch of horizontal dashed lines, from this configuration the only possible update is to create a worm with one particle in a given worldline, and trough the space shift and time shift updates the particle will therefore move in the sites of the lattice. 


\subsubsection{Advantages of the Worm algorithm}

The updates described above are all local and allow to draw/erase any line, and jump between the sites. Although only configurations belonging to the $C P$ space contribute to the evaluation of the partition function, by using the enlarged configuration space $C P+C P_{\mathrm{g}}$ the intermediate configurations with one disconnected loop allow to efficiently collect statistics for the Green function. For an algorithm working in the $C P$ space only, instead, collecting statistics for the Green function results computationally very expensive.

Another advantage of the WA is that it does not suffer from critical slowing down in the vicinity of a critical point. In the critical region, a system develops long range correlations, and in most cases an algorithm based on local updates results very inefficient in simulating such a system for which the relevant degrees of freedom are non-local, and it results in the divergence of the autocorrelation time with the system size. Although the WA performs local updates, it overcomes this problem by using the drawing and erasing updating procedures through the worm ends, which are directly linked to the critical modes (long range order in $G(j, \tau)$ ). This turns out to be very efficient in generating independent configurations also in the critical region.

The WA is also efficient in sampling topologically different configurations and configurations which are separated by an energy barrier, which is a necessary condition in order to maintain ergodicity. An example of two topologically different configurations is shown in Fig. 8.5, where a one-dimensional system with one particle (worldline) is considered. Periodic boundary conditions in time and space apply, i.e. the system is a torus where the bottom and top facets of the cylinder are glued together. Fig. 8.5(a) represents a configuration with zero winding numbers, i.e. the worldline does not 'wind' in imaginary time. Fig. 8.5(d), instead, represents a configuration with one winding number, i.e. the worldline winds once in imaginary time. An algorithm based on local updates which only works in the $C P$ space would not allow to sample configurations with different winding numbers, unless a global update which introduces a winding number at once, is introduced. The WA, instead, can easily go from configuration of Fig. 8.5(a) to configuration Fig. 8.5(d) (see a sketch in Fig. 8.5(b)-(c)).

Being able to sample configurations with different winding numbers is crucial in order to simulate $S F$ systems. It was shown in [84], that the superfluid stiffness can be extracted from the statistics of winding numbers:

$$
\rho_{s}=\frac{T\left\langle\mathbf{W}^{2}\right\rangle}{d L^{d-2}},
$$

where $T$ is the temperature, $L$ the system size, $d$ the dimensionality, and $\mathbf{W}^{2}=\sum_{i=1}^{d} W_{i}^{2}$. 
a)

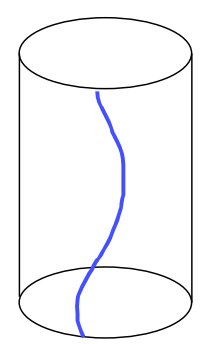

b)

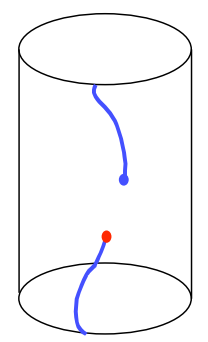

c)

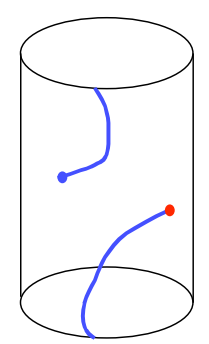

d)

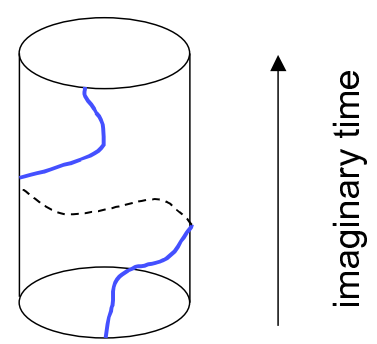

Figure 8.5: One-dimensional system with (a) zero and (d) one winding num$\operatorname{ber}(\mathrm{s})$. (b)-(c) sketch on how the WA is able to go from (a) to (d). 


\section{Chapter 9}

\section{Quantum Monte Carlo studies of dipolar gases}

Quantum Monte Carlo is one of the most powerful methods we have to study equilibrium properties of strongly interacting many-body quantum systems. In the literature, there is a large amount of work devoted to the study of dipolar gases with Quantum Monte Carlo techniques. From self-assembled floating lattices, provided by trapped polar molecules [38], to the possibility of tuning, and shaping the long-range interaction potential of polar molecules [39], to self-organized mesoscopic structures of matter waves in zigzag chains [29], to the spectrum of the elementary excitation that can exhibit a roton minimum $[31,30]$, to the emergence of an emulsion phase in triangular lattices [32]. The ones listed above are just a few of the outstanding properties of dipolar gases, which have been investigated with various Monte Carlo techniques.

The results presented in this thesis are based on the Path Integral Monte Carlo and the Worm algorithm, which, as discussed in Sec. 8.2.2, turns out to be very efficient in simulating systems presenting $S F$ phases. Particularly for Hubbard models, because of its efficiency in collecting statistics for the Green function, the WA has proven to be a very powerful tool to investigate the ground state properties of such systems. In fact, as we will explain in the next section, from the behavior of the Green function in imaginary time, one can extract informations about the insulating phases of the ground state of the system, which are typical of Hubbard models. 


\subsection{Zero momentum Green function and the particle-hole excitations}

Before discussing our main results in Sec 9.2, it is instructive to introduce a rather technical but useful quantity. The zero momentum Green function is defined as the sum

$$
G(\vec{p}=0, \tau)=\sum_{j} G(j, \tau)
$$

over all sites $j$ of the lattice, and is particularly useful to determine the ground state phase diagram of various types of Bose-Hubbard Hamiltonians. In particular for the dipolar Hamiltonian (8.7) the phase boundaries of the Mott insulating lobes are readily found from the exponential decay of the Green function Eq. (9.1) with the imaginary time [83]. In fact, for any values of the tunneling coefficient $J$ and of the chemical potential $\mu$ inside a given insulating lobe, it is straightforward to determine the dispersion relation for the single particle and single hole excitations by using a Lehman expansion and extrapolation in the $\tau \rightarrow \infty$ limit. In this limit one finds that the zero momentum Green function in the momentum space behaves as follows

$$
G(\vec{p}=0, \tau) \longrightarrow \begin{cases}e^{-\tau E_{\mathrm{P}}(\mu, J)}, & \tau \rightarrow+\infty \\ e^{+\tau E_{\mathrm{H}}(\mu, J)}, & \tau \rightarrow-\infty\end{cases}
$$

where $E_{\mathrm{P}}(\mu, J)$ and $E_{\mathrm{H}}(\mu, J)$ are the single particle and single hole excitation energies at a given $\mu$ and $J$ inside the lobe. At the lobe boundaries $\mu=\mu_{\mathrm{b}}$, one of the two excitations either $E_{\mathrm{P}}\left(\mu_{\mathrm{b}}, J\right)$ or $E_{\mathrm{H}}\left(\mu_{\mathrm{b}}, J\right)$ vanishes and only at the critical point given by tip of the insulating lobe at $\left(\mu_{\mathrm{c}}, J_{\mathrm{c}}\right)$ both of the excitations are zero. Therefore, the phase boundaries are easily found by searching the values of $(\mu, J)$ for which one or both the energy of the elementary excitations vanish.

\subsection{Incompressible and supersolid phases}

In our work [86], we have studied the ground state properties of dipolar hardcore Bosons confined in a 2D square lattice of linear size $L$, satisfying periodic boundary conditions. The system is described by the extended Bose-Hubbard Hamiltonian (8.7), where no cut-off in the dipolar interaction potential is used [86]. The incompressible and supersolid phases are both characterized by a finite value of the structure factor, defined as

$$
S(\mathbf{k})=\sum_{\mathbf{r}, \mathbf{r}^{\prime}} \frac{\left\langle n_{\mathbf{r}} n_{\mathbf{r}^{\prime}}\right\rangle}{N} e^{i \dot{\mathbf{k}}\left(\mathbf{r}-\mathbf{r}^{\prime}\right)}
$$


with $\mathbf{k}$ the reciprocal lattice vector, $n_{\mathbf{r}}$ the density at position $\mathbf{r}$, and $N$ the total number of particles. While for the incompressible phases the superfluid fraction vanishes $\rho_{s}=0$, the supersolid phase is characterized by a finite value of $\rho_{s}$, indicating the presence of superfluid.

\subsubsection{Homogeneous case}

Our main results for $\Omega=0$ are summarized in Fig. 9.1, where we show the zero temperature phase diagram of the system, in the $J$ vs. $\mu$ plane, in the range $J / V>0.02$, and $1<\mu / V<6$ indicated by the unshaded area.

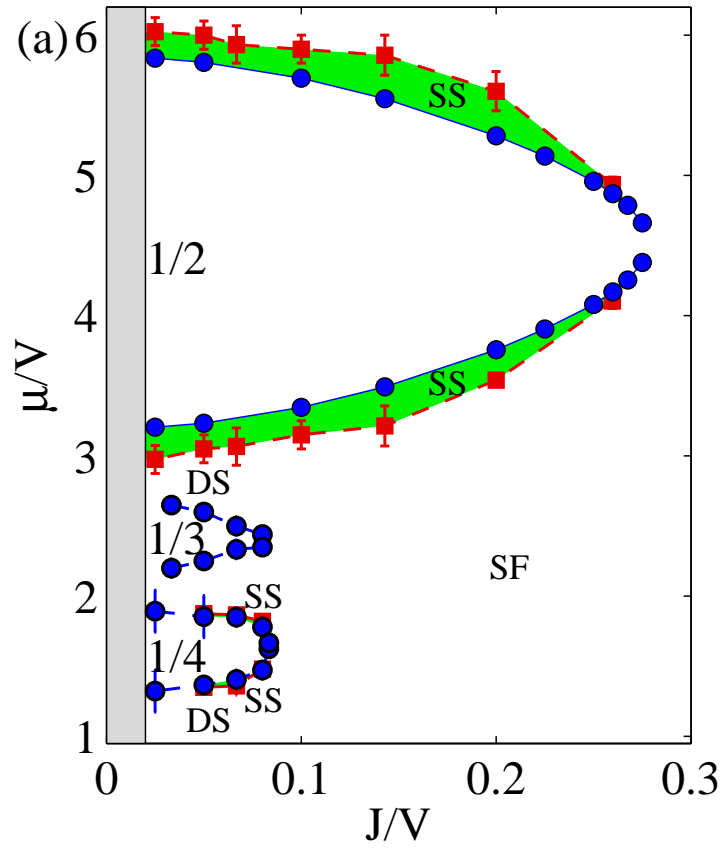

(b)

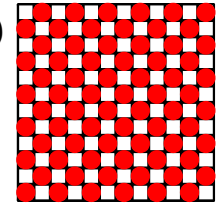

(c)

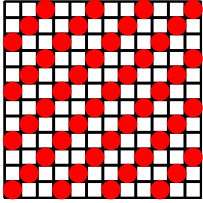

(d)

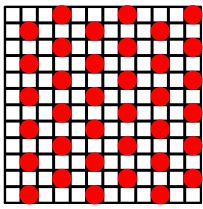

Figure 9.1: Phase diagram corresponding to the Hamiltonian Eq. (8.7) as a function of $\mu$ and $J$ at zero temperature. Lobes: Mott solids (densities indicated); SS: supersolid phase; SF: superfluid phase. DS: parameter region where devil' s staircase is observed. Panels (b-d): sketches of the groundstate configuration for the Mott solids in panel (a), with $\rho=1 / 2,1 / 3$ and $1 / 4$, respectively.

For finite $J$, three main solid Mott lobes emerge with filling factor $\rho=1 / 2$, $1 / 3$, and $1 / 4$, named checkerboard (CB), stripe (ST), and star (SR) solids, respectively. The corresponding groundstate configurations are sketched in 
panels (b-d). The boundaries of the Mott lobes have been calculated from the zero momentum Green function, as described in Sec. 9.1, for linear system sizes up to $L=20$ (CB and SR lobes), and from $\mu$-curves with sizes up to $L=24$ (ST lobe). We find that the CB solid is the most robust against hopping and doping, and thus it extends furthest in the $J$ vs. $\mu$ plane

For large enough $J / V$, the low-energy phase is superfluid (SF), for all $\mu$. At intermediate values of $J / V$, however, we find that by doping the Mott solids either with vacancies (removing particles) or interstitials (adding extra particles) a supersolid phase (SS) can be stabilized, with coexisting superfluid and crystalline orders. Instead, we find no evidence of SS in the absence of doping. The green shaded area above and below the CB lobe boundaries in Fig. 9.1, correspond to a SS obtained by doping the CB crystal with interstitials, and vacancies respectively. Remarkably, the long-range interactions stabilizes the supersolid in a wide range of parameters, in fact for $1 \mathrm{NN}$ or $2 \mathrm{NN}$ in the range of the dipolar interaction, no stable CB SS was found for $\rho<1 / 2[12]$.

Interestingly, we find evidence for incompressible phases in addition to those corresponding to the lobes in Fig. 9.1. This is shown in Fig. 9.2, where the particle density $\rho$ is plotted as a function of the chemical potential $\mu$.
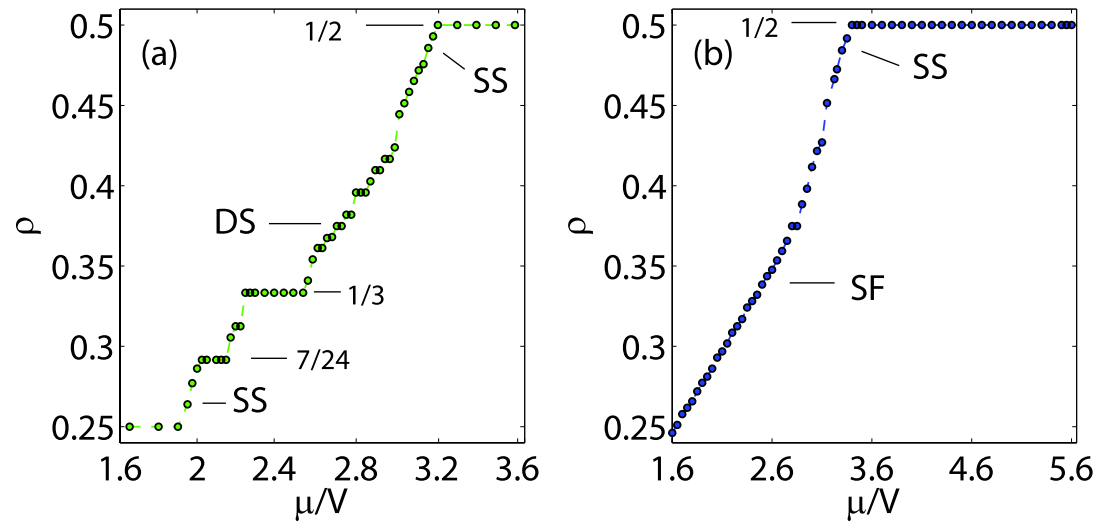

Figure 9.2: $\rho$ vs. $\mu$. (a): Solids and SS for a system with linear size $L=12$ and $J / V=0.05$. Some $\rho$ are indicated. (b): SF and vacancy-SS for $L=16$ and $J / V=0.1$.

In Fig. 9.2, a continuous increase of $\rho$ as a function of $\mu$ signals a compressible phase, while a solid phase is characterized by a constant $\rho$ for increasing $\mu$. Panel (a), corresponding to $J / V=0.05$, shows a series of large constantdensity plateaux connected by a progression of smaller steps and regions of 
continuous increase of $\rho$. Here, the main plateaux correspond to the Mott lobes of Fig. 9.1, while the other steps correspond to incompressible phases, with a fixed, integer, number of particles. This progression of steps is an indication of a devil's-like staircase in the density, which was discussed in [13] for a one dimensional system. Instead, for $J / V=0.1$ in panel (b), no evidence of such a phase is found.

\subsubsection{Finite temperature}

In [86] we have studied the melting of the supersolid into a normal phase with increasing temperature $T$, for the case of vacancy supersolidity below the CB solid, with $J / V=0.1$. Fig. 9.3 shows the superfluid fraction $\rho_{s}$, and the structure factor for the CB solid $S(\pi, \pi)$ as a function of $T$. We find that the melting of the supersolid proceeds through two successive transitions. First, the supersolid melts into a liquid-like phase reminiscent of a liquid crystal, with zero $\rho_{s}$ and finite $S(\pi, \pi)$. The drop of $\rho_{s}$ for $T \simeq 0.1 J$ in Fig. 9.3 signals a transition of the Kosterlitz-Thouless (KT) type, with critical melting temperature $T_{\mathrm{KT}}=\pi \rho_{s} \hbar^{2} \rho / 2 m$, and $m=1 / 2 J a^{2}$, with $a$ being the lattice spacing, and $\rho$ the filling factor. Upon further increasing temperature, we find that the static structure factor drops to zero for $T \simeq J=2.6 \mathrm{~V}$. In panel (b) we show that this is consistent with an Ising-type transition, by plotting the expected scaling for $S(\pi, \pi)$ in two dimensions (here, $2 \beta \nu=1 / 4$ ).

\subsubsection{Harmonic confinement}

The recent experimental achievement of single-site addressability in optical lattices using electron and optical microscopy allows for a direct, in-situ, observation of particle positions and particle-particle correlations in experiments $[64,85]$. Thus, the key observables for present and future experiments are the in-situ density distribution and particle correlations, from which the phases above can be detected. The question is how the phases described in Fig. 9.1 will be seen in an experiment. Here, we provide snapshots of particle configurations for realistic experimental situations with $N \sim 10^{3}$ particles trapped with harmonic confinement, and small finite $T$.

In Fig. 9.4 we show snapshots of the spatial density distribution in the lattice (shown is a single quadrant). Each circle corresponds to a different site, and its radius is proportional to the local density.

In panels (a) and (b), $\mu$ has been chosen such that particles at the trap center are in the $\mathrm{CB}$ phase, with very small $T$. The density profile shows 

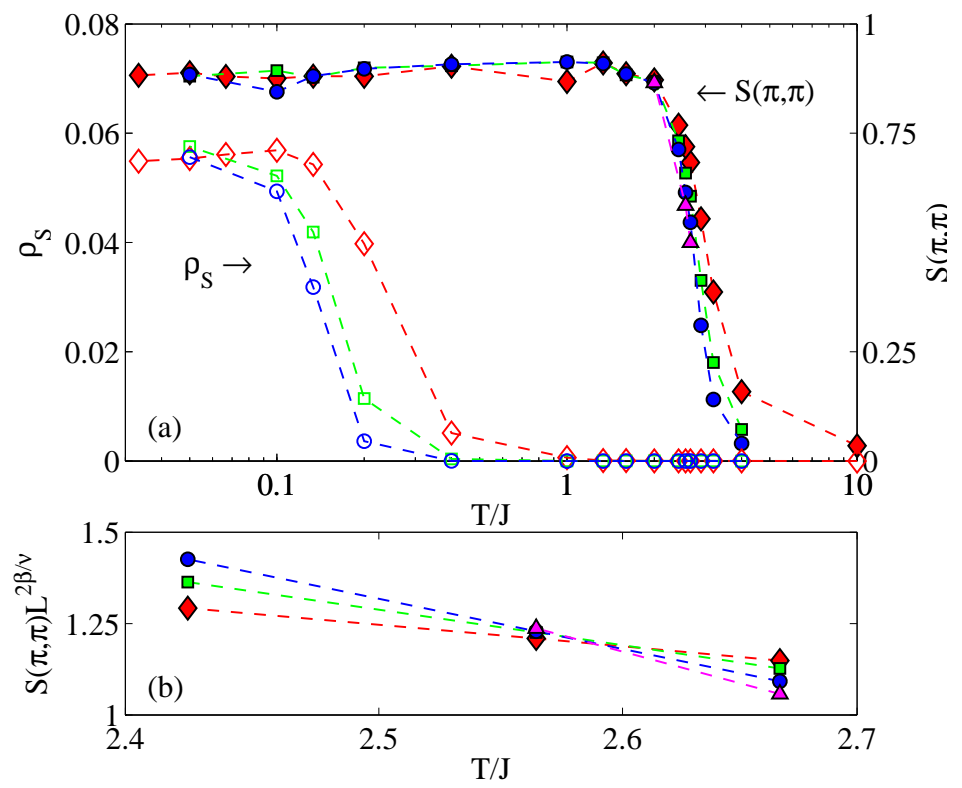

Figure 9.3: Finite- $T$ melting of SS at $J / V=0.1$ : (a) temperature $T$ versus $\rho_{s}$ (empty symbols) and $S(\pi, \pi)$ (full symbols), for linear size systems of $L=8,12,16$ and 20 (diamonds, squares, dots, and triangles, respectively); (b) $T$ vs. $S(\mathbf{k}) L^{2 \beta / \nu}$, with $2 \beta / \nu=1 / 4$.

a wedding-cake structure, with concentric Mott-lobes with density $\rho=1 / 2$ and $1 / 4$. However, while the system parameters are the same in both figures, panel (a) shows regular CB and SR patterns, while in panel (b) extended defects are present in the $\mathrm{CB}$ phase and the $\mathrm{SR}$ is barely visible. This is due to the different preparation of the states in panels (a) and (b). In fact, in panel (a) we performed temperature annealing of the system prior to taking the snapshot, while this was not done in panel (b). We find that the defects in (b) reflect the existence of a large number of low-energy metastable states, which are a direct consequence of the long-range nature of the interactions, and will be of relevance for experiments.

SS and ST phases are shown in panels (c) and (d), respectively. In panel (c), $\mu$ has been chosen to realize an extended vacancy-SS region, surrounded by a SF. We notice that here a finite $T=0.1 J$ has been chosen, compatible with the existence of the SS phase. The density-distribution in the vacancySS looks similar to the ordered CB phase, even without annealing. Selfannealing is in fact here enabled by the (small) superfluid component of the SS phase. Small coherence peaks will be present in time-of-flight experiments, 

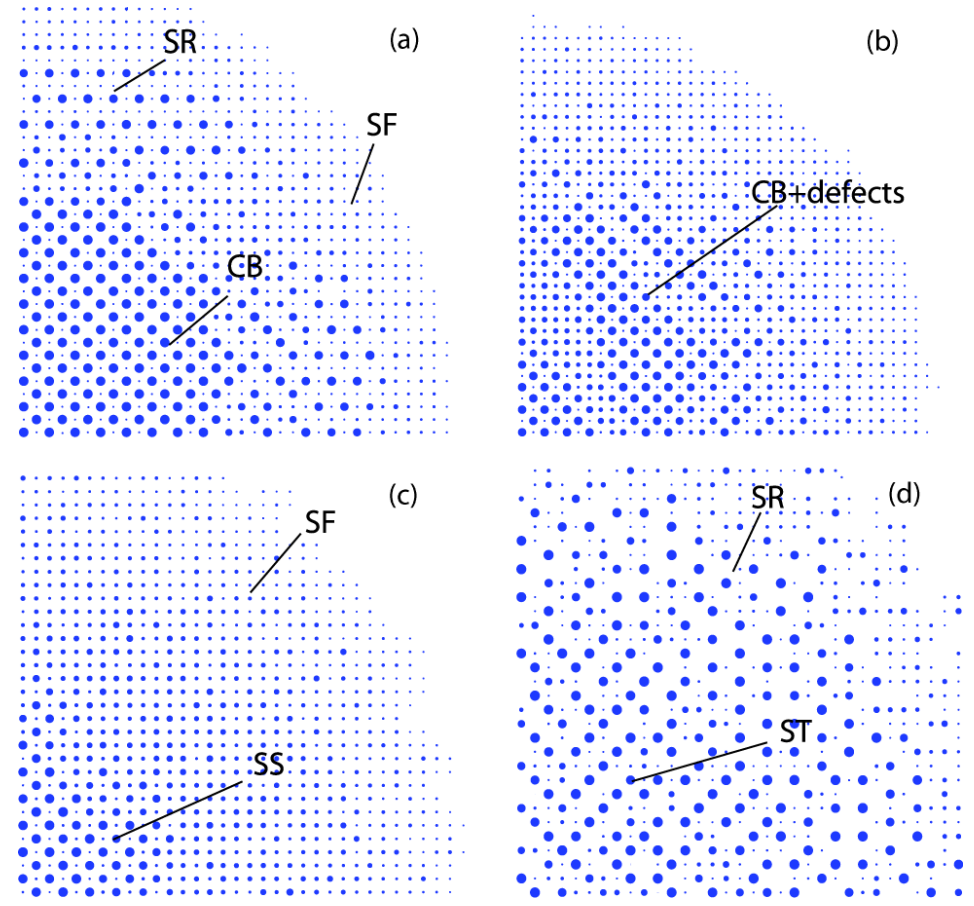

Figure 9.4: Spatial density profile in 2D for $N \simeq 1000$ particles in a harmonic potential. Phases are indicated. (a-b) $V / J=15, \mu / J=55, \Omega / J=0.05$ and $T / J=0.0377$; (c) $V / J=5, \mu / J=19, \Omega / J=0.01$ and $T / J=0.1$; (d) $V / J=20, \mu / J=51, \Omega / J=0.04$ and $T / J=0.25$.

allowing for a clear determination of this phase. Panel (d) shows a disordered ST-phase at the center, surrounded by an extended Mott-shell with $\rho=1 / 4$. The disorder in this case is a result of both finite $T / J=0.25$ and the fact that the ST solid is less robust towards quantum and classical fluctuations compared to the $\mathrm{CB}$ and SR ones.

These exact results for $\Omega \neq 0$ confirm that the phase-diagram Fig. 9.1 is the key to predict and interpret experimental observables, assuming a local density approximation. 


\section{Chapter 10}

\section{Conclusions}

In this thesis we have studied Bose-Hubbard (BH) models with dipolar interactions. In $[57,58]$ we have studied a single component gas of dipolar Bosons in a two-dimensional (2D) optical lattice, where the dipoles are polarized perpendicularly to the 2D plane, resulting in an isotropic repulsive interaction. With a mean-field approximation based on a Gutzwiller Ansatz, we have shown that such a system possesses many almost degenerate metastable states, similarly to a disordered system, and we have shown that the dipoledipole interaction is responsible for the appearance of these states. We have studied in detail the fate of these metastable states, showing how they can be prepared on demand, we have discussed current experimental techniques that may be able to detect the metastable states, and we have calculated their lifetime due to tunneling. We have studied the ground state of the system and found the presence of insulating checkerboard-like states with fractional filling factors $\nu$, and by using Quantum Monte Carlo methods (the Worm algorithm) in [86] we have confirmed this prediction. Moreover, we have found evidences for a Devil' s staircase in the ground state, which was previously predicted in the phase diagram of a one-dimensional geometry [13] but not in 2D. Interestingly, we have found supersolid regions in the ground state, obtained by doping the solids either with particles or vacancies.

In [74], we have studied a system composed of two 2D layers in which the dipoles are polarized perpendicularly to the planes. The dipolar interaction is therefore repulsive for particles laying on the same plane, while it is attractive for particles at the same lattice site on different layers. Our mean-field calculations have shown that particles pair into composites, and we have demonstrate the existence of the novel Pair Super Solid (PSS) quantum phase. It would be interesting to verify the extension of the PSS phase by considering more nearest neighbors in the dipolar interactions, both with mean-field and Quantum Monte Carlo methods. 
Another interesting direction of investigation may be to study how the previous situation changes by adding more layers. For example, in a three layers structure, it could be interesting to verify whether the pairing behavior still survives or if the system is dominated by a different physics, e.g. like the formation of triplets.

The system we are currently studying, namely a 2D lattice where the dipoles are free to point in both directions perpendicularly to the plane, has shown many encouraging results. Within a mean-field approach, we have found magnetic phases where the ground state presents ferromagnetic or anti-ferromagnetic ordering. For large enough tunneling, we have found evidence for the existence of a Counterflow Super Solid (CSS) quantum phase. Based on current experimental techniques with $\mathrm{OH}$ molecules, we have proposed a possible realization of this system in the laboratory. Our theoretical study on this system is still on-going, and detailed mean-field calculations providing the validity of the CSS phase have to be done. As well as for the two-layers system described above, increasing the number of nearest neighbors in the dipolar interactions could be a possible direction of investigation. Finally, changing the geometry of the optical lattice could lead to interesting phenomena, as frustration, which may happen in a triangular lattice.

As previously stated, our predictions have direct experimental consequences, and we hope that they will be soon checked in experiments with ultracold dipolar atomic and molecular gases. 


\section{Acknowledgments}

It is a great pleasure for me to thank all the people in the quantum optics theory group of ICFO, present and past members. I spent four beautiful years of my life, I have learned, and I have changed, and it is from my heart that I thank all the group. I especially thank Chiara Menotti, and Maciek Lewenstein for their constant support, and patience, they showed me during these years, the result of which is not only in this thesis work. I thank Barbara Capogrosso-Sansone and Guido Pupillo, for their guide in the Quantum Monte Carlo work, and Peter Zoller for the kind hospitality while I was in Innsbruck.

This thesis was partially written at the Indian Association for the Cultivation of Science, in Calcutta, while I was visiting Krishnendu Sengupta. The worm hospitality he showed me is unique, and I thank him together with all the people of the theoretical physics department.

Outside of ICFO there are many people who helped me during these years. A special thanks goes to Barbara, who supported me all the time, and knows almost all the people in the group without having met them. To my friend Antoni, with whom I have shared very funny situations, and last, but not least, to my family. 


\section{Appendix A}

\section{Spectrum of excitations}

The low-lying excitation energies are creating particles $(\mathrm{P})$ and holes $(\mathrm{H})$ in a given metastable configuration. For every site $i$, at $J=0$ the excitation energies are given by $E_{\mathrm{P}}^{i}=-\mu+U n_{i}+V_{\text {dip }}^{1, i}$ and $E_{\mathrm{H}}^{i}=\mu-U\left(n_{i}-1\right)-V_{\text {dip }}^{1, i}$, where $n_{i}$ is the density at site $i$. Clearly the hole excitation for $n_{i}=0$ is unphysical. At finite $J$, the excitation spectrum $\omega(\mathbf{k})$ of a metastable configuration, is given by the small fluctuations $\delta f_{\mathrm{n}}^{(i)}(t)$ around the unperturbed metastable state coefficients $\bar{f}_{\mathrm{n}}^{(i)}$. In a Mott state with exactly $m$ particles at site $i$, the only non-zero coefficients are given by $\bar{f}_{m}^{(i)}$. Writing $f_{\mathrm{n}}^{(i)}=\bar{f}_{\mathrm{n}}^{(i)}+\delta f_{\mathrm{n}}^{(i)}(t)$ in Eq. (2.6), and taking into account only linear terms in the fluctuations, we get

$$
\begin{aligned}
i \delta \dot{f}_{\mathrm{n}}^{(i)} & \simeq-J\left[\bar{\varphi}_{i} \sqrt{n} \bar{f}_{n-1}^{(i)}+\bar{\varphi}_{i}^{*} \sqrt{n+1} \bar{f}_{n+1}^{(i)}\right] \\
& +\left[\frac{U}{2} n(n-1)+n V_{\mathrm{dip}}^{1, i}-\mu n-\chi_{m}^{(i)}\right] \delta f_{\mathrm{n}}^{(i)},
\end{aligned}
$$

where $\bar{\varphi}_{i} \simeq \sum_{\langle j\rangle_{i}} \sum_{n} \sqrt{n+1}\left(\bar{f}_{\mathrm{n}}^{(j) *} \delta f_{\mathrm{n}+1}^{(j)}+\bar{f}_{\mathrm{n}+1}^{(j)} \delta f_{\mathrm{n}}^{(j) *}\right)$, and $\chi_{\mathrm{m}}^{(i)}=\frac{U}{2} m(m-$ 1) $+m V_{\text {dip }}^{1, i}-\mu m$ is an extra phase that we have introduced to eliminate the rotating phase of the $\bar{f}_{\mathrm{m}}^{(i)}$ coefficients. The only non-trivial terms in Eq. (A.1) are therefore

$$
\begin{aligned}
& i \delta f_{\mathrm{m}-1}^{\dot{(i)}}=E_{\mathrm{H}}^{i} \delta f_{\mathrm{m}-1}^{(i)}-J \sqrt{m} \bar{\varphi}_{i}^{*} \\
& i \delta f_{\mathrm{m}+1}^{(i)}=E_{\mathrm{P}}^{i} \delta f_{\mathrm{m}+1}^{(i)}-J \sqrt{m+1} \bar{\varphi}_{i}
\end{aligned}
$$

and their complex conjugates. It is convenient to study Eq. (A.2) and their complex conjugates in the Fourier domain with $\delta f_{\mathrm{n}}^{(i)}(t)=\sum_{k} e^{i \mathbf{k} \cdot \mathbf{x}^{(i)}} a_{n}^{(i)}(\mathbf{k}, t)$, $\mathbf{x}^{(i)}$ being the $2 \mathrm{D}$ vector pointing at site $i$. After simple algebra one finds the 
Fourier modes to fulfill

$$
\begin{aligned}
i \dot{a}_{\mathrm{m}-1}^{(i)}(\mathbf{k}, t) & =E_{\mathrm{H}}^{i} a_{\mathrm{m}-1}^{(i)}(\mathbf{k}, t) \\
& -J \sqrt{m} \sum_{\langle j\rangle_{i}}\left[\sqrt{m+1} a_{\mathrm{m}+1}^{(j) *}(-\mathbf{k}, t)\right. \\
& \left.+\sqrt{m} a_{\mathrm{m}-1}^{(j)}(\mathbf{k}, t)\right] e^{i \mathbf{k} \cdot \mathbf{d}^{\langle j\rangle}} \\
i \dot{a}_{\mathrm{m}+1}^{(i)}(\mathbf{k}, t) & =E_{\mathrm{P}}^{i} a_{\mathrm{m}+1}^{(i)}(\mathbf{k}, t) \\
& -J \sqrt{m+1} \sum_{\langle j\rangle_{i}}\left[\sqrt{m+1} a_{\mathrm{m}+1}^{(j)}(\mathbf{k}, t)\right. \\
& \left.+\sqrt{m} a_{\mathrm{m}-1}^{(j) *}(-\mathbf{k}, t)\right] e^{i \mathbf{k} \cdot \mathbf{d}^{\langle j\rangle}},
\end{aligned}
$$

with $\mathbf{d}^{\langle j\rangle}=\{ \pm(d, 0), \pm(0, d)\}$ being the vectors of nearest neighbors in the lattice, and $d$ the lattice spacing. We look for stationary solutions of Eqs. $(\mathrm{A} .3, \mathrm{~A} .4)$ with the ansatz $a_{\mathrm{n}}^{(i)}(\mathbf{k}, t)=u_{\mathrm{n}}^{(i)}(\mathbf{k}) e^{-i \omega(\mathbf{k}) t}+v_{\mathrm{n}}^{(i)}(\mathbf{k}) e^{i \omega(\mathbf{k}) t}$. For every site $i$ of the elementary cell, Eqs. (A.3,A.4) become

$$
\begin{aligned}
{\left[E_{\mathrm{H}}^{i}-\omega(\mathbf{k})\right] u_{\mathrm{m}-1}^{(i)}(\mathbf{k}) } & -J \sqrt{m} \sum_{\langle j\rangle_{i}}\left[\sqrt{m+1} v_{\mathrm{m}+1}^{(j) *}(-\mathbf{k})\right. \\
& \left.+\sqrt{m} u_{\mathrm{m}-1}^{(j)}(\mathbf{k})\right] e^{i \mathbf{k} \cdot \mathbf{d}^{\langle j\rangle}}=0 \\
{\left[E_{\mathrm{P}}^{i}+\omega(\mathbf{k})\right] v_{\mathrm{m}+1}^{(i) *}(\mathbf{k}) } & -J \sqrt{m+1} \sum_{\langle j\rangle_{i}}\left[\sqrt{m+1} v_{\mathrm{m}+1}^{(j) *}(-\mathbf{k})\right. \\
& \left.+\sqrt{m} u_{\mathrm{m}-1}^{(j)}(\mathbf{k})\right] e^{i \mathbf{k} \cdot \mathbf{d}^{\langle j\rangle}}=0, \\
& -J \sqrt{m+1} \sum_{\left.E_{\mathrm{P}}^{i}-\omega(\mathbf{k})\right] u_{\mathrm{m}+1}^{(i)}(\mathbf{k})}\left[\sqrt{m+1} u_{\mathrm{m}+1}^{(j)}(\mathbf{k})\right. \\
& \left.+\sqrt{m} v_{\mathrm{m}-1}^{(j) *}(-\mathbf{k})\right] e^{i \mathbf{k} \cdot \mathbf{d}^{\langle j\rangle}}=0, \\
& -J \sqrt{m} \sum_{\langle j\rangle_{i}}\left[\sqrt{m+1} u_{\mathrm{m}+1}^{(j)}(\mathbf{k})\right. \\
{\left[E_{\mathrm{H}}^{i}+\omega(\mathbf{k})\right] v_{\mathrm{m}-1}^{(i) *}(-\mathbf{k}) } & \left.\sqrt{m} v_{\mathrm{m}-1}^{(j) *}(-\mathbf{k})\right] e^{i \mathbf{k} \cdot \mathbf{d}^{\langle j\rangle}}=0 .
\end{aligned}
$$

This set of $4 N^{2}$ equations can be reduced depending on the symmetry of the density distribution, like in the case of the checkerboard where only two sites are relevant. Eqs. (A.5) can be written in a matrix form, $M\left(\begin{array}{l}\mathbf{u} \\ \mathbf{v}^{*}\end{array}\right)=0$, and have non-trivial solution only if $\operatorname{det}[M]=0$. The excitation spectrum is then given by the positive solutions of the last equation. In Fig. A.1, 
we show the lowest excitation branch of the four metastable configurations of Fig. 3.2, for $\mu=3.3 U_{N N}, J=0.1 U_{N N}$ and $k_{x} d=k_{y} d=k / \pi$, in the first Brillouin zone. The thick line is for the (CB) state, the dashed, dashdotted and dotted lines are for (I), (IIa) and (IIb) states respectively. At the boundaries of the insulating lobes the excitation spectrum $\omega(\mathbf{k}=0)$ goes to zero.

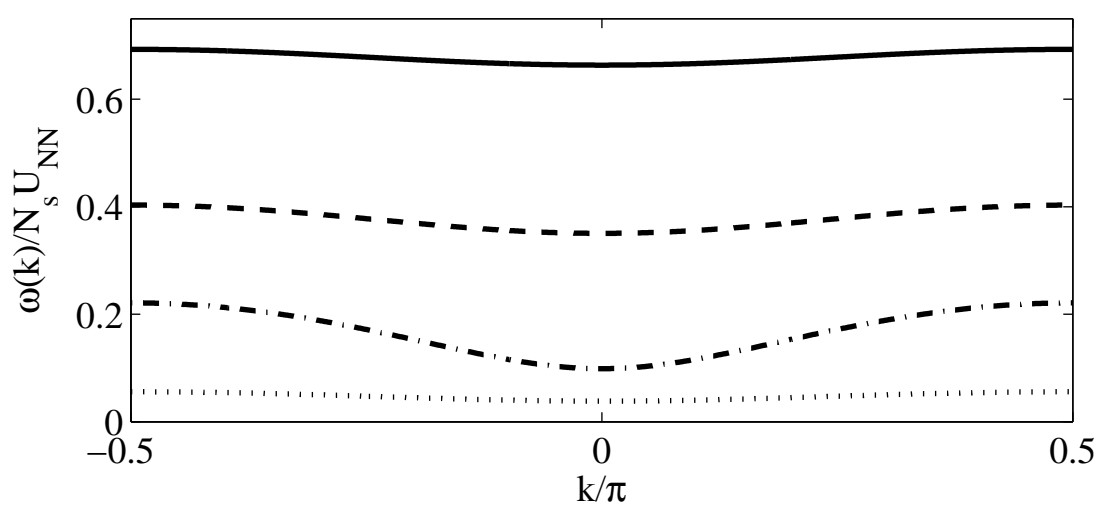

Figure A.1: Lowest excitation spectrum of metastable state (GS) (thick), (I) (dashed), (IIa) (dash-dotted) and (IIb) (dotted) of Fig. 3.2 calculated for $\mu=3.3 U_{N N}, J=0.1 U_{N N}$, and four nearest neighbors in the dipolar interaction range. 


\section{Bibliography}

[1] M. P.A. Fisher, P. B. Weichman, G. Grinstein, D. S. Fisher, Boson localization and the superfluid-insulator transition. Physical Review B, 40, 1, 1989.

[2] M. Greiner, O. Mandel, T. Esslinger, T. W. Hänsch, and I. Bloch, Quantum phase transition from a superfluid to a Mott insulator in a gas of ultracold atoms. Nature 415, 39-44 (2002).

[3] A. Griesmaier, J. Werner, S. Hensler, J. Stuhler and T. Pfau, BoseEinstein condensation of chromium. Physical Review Letters 94, 160401 (2005).

[4] J. Stuhler, A. Griesmaier, T. Koch, M. Fattori, T. Pfau, S. Giovanazzi, P. Pedri, and L. Santos, Observation of Dipole-Dipole Interaction in a Degenerate Quantum Gas. Physical Review Letters 95, 150406 (2005).

[5] S. Hensler, J. Werner, A. Griesmaier, P.O. Schmidt, A. Görlitz, T. Pfau, S. Giovanazzi and K. Rzążewski, Dipolar relaxation in an ultra-cold gas of magnetically trapped chromium atoms. Applied Physics B: Lasers and Optics, Volume 77, Number 8 / December, 2003.

[6] K.-K. Ni, S. Ospelkaus, M. H. G. de Miranda, A. Pe'er, B. Neyenhuis, J. J. Zirbel, S. Kotochigova, P. S. Julienne, D. S. Jin, and J. Ye, A High Phase-Space-Density Gas of Polar Molecules. Science 322, 231-235 (2008).

[7] S. Ospelkaus, A. Pe'er, K.-K. Ni, J. J. Zirbel, B. Neyenhuis, S. Kotochigova, P. S. Julienne, J. Ye, D. S. Jin, Efficient state transfer in an ultracold dense gas of heteronuclear molecules. Nature Physics 4, 622-626 (2008).

[8] J. J. Zirbel, K.-K. Ni, S. Ospelkaus, T. L. Nicholson, M. L. Olsen, P. S. Julienne, C. E. Wieman, J. Ye, and D. S. Jin, Heteronuclear molecules in an optical dipole trap. Physical Review A 78, 013416/1-7 (2008). 
[9] K. Góral, L. Santos, and M. Lewenstein, Quantum Phases of Dipolar Bosons in Optical Lattices. Physical Review Letters, 88, 170406 (2002).

[10] D. L. Kovrizhin, G. V. Pai, and S. Sinha, Density wave and supersolid phases of correlated bosons in an optical lattice. Europhysics Letters, 72, 162-168 (2005).

[11] G. G. Batrouni and R. T. Scalettar, Phase Separation in Supersolids. Physical Review Letters 84, 1599 (2000).

[12] P. Sengupta, L. P. Pryadko, F. Alet, M. Troyer, and G. Schmid, Supersolids versus Phase Separation in Two-Dimensional Lattice Bosons. Physical Review Letters 94, 207202 (2005).

[13] F. J. Burnell, Meera M. Parish, N. R. Cooper, and S. L. Sondhi, Devil's staircases and supersolids in a one-dimensional dipolar Bose gas. Physical Review B 80, 174519 (2009).

[14] M. Lewenstein, A. Sanpera, V. Ahufinger, B. Damski, A. Sen(DE), and U. Sen, Ultracold atomic gases in optical lattices: mimicking condensed matter physics and beyond. Advances in Physics, Vol. 56, No. 2, MarchApril 2007, 243-379.

[15] I. Bloch, Quantum gases in optical lattices. Physics World 1725 (2004).

[16] L. Pitaevskii and S. Stringari, Bose-Einstein Condensation. Oxford University Press 2003.

[17] C. J. Pethick \& H. Smith, Bose-Einstein Condensation in Dilute Gases, second edition. Cambridge University Press, 2008.

[18] N. H. March, W. H. Young, and S. Sampanthar, The Many-Body Problem in Quantum Mechanics. Cambridge University Press, 1967.

[19] A. L. Fetter, and J. D. Walecka, Quantum Theory of Many-Particle Systems. Dover Publications, 1971.

[20] A. Auerbach, Interacting Electrons and Quantum Magnetism. SpringerVerlag New York, 1994.

[21] F. H. L. Essler, H. Frahm, F. Gömann, A. Klümper, and V. E. Korepin, The One-Dimensional Hubbard Model. Cambridge University Press, 2005.

[22] D. Jacksch, C. Bruder, J. I. Cirac, C. W. Gardiner, and P. Zoller, Cold Bosonic Atoms in Optical Lattices. Physical Review Letters, 81, 3108 (1998). 
[23] M. A. Baranov, Ł. Dobrek, and M. Lewenstein, Superfluidity of Trapped Dipolar Fermi Gases. Physical Review Letters 92, 250403 (2004).

[24] A. Derevianko, Anisotropic pseudopotential for polarized dilute quantum gases. Physical Review A 67, 033607 (2003).

[25] A. Derevianko, Revised Huang-Yang multipolar pseudopotential. Physical Review A 72, 044701 (2005).

[26] A. Derevianko, Erratum: Anisotropic pseudopotential for polarized dilute quantum gases [Phys. Rev. A 67, $03360^{77}$ (2003)]. Physical Review A 72, 039901 (2005).

[27] G. E. Astrakharchik, J. Boronat, and J. Casulleras, I. L. Kurbakov and Yu. E. Lozovik, Weakly interacting two-dimensional system of dipoles: Limitations of the mean-field theory. Physical Review A 75, 063630 (2007).

[28] S. Giorgini, J. Boronat, and J. Casulleras, Ground state of a homogeneous Bose gas: A diffusion Monte Carlo calculation. Physical Review A 60, 5129 (1999).

[29] G. E. Astrakharchik, G. Morigi, G. De Chiara, and J. Boronat, Ground state of low-dimensional dipolar gases: Linear and zigzag chains. Physical Review A 78, 063622 (2008).

[30] G.E. Astrakharchik, J. Boronat, I. L. Kurbakov, and Yu. E. Lozovik, Quantum phase transition in a two-dimensional system of dipoles. Physical Review Letters 98, 060405 (2007).

[31] F. Mazzanti, R. E. Zillich, G. E. Astrakharchik, and J. Boronat, Dynamics of a Two-Dimensional System of Quantum Dipoles. Physical Review Letters 102, 110405 (2009).

[32] L. Pollet, J. D. Picon, H. P. Büchler, and M. Troyer, Supersolid phase with cold polar molecules on a triangular lattice. arXiv:0906.2126.

[33] S. Pilati, K. Sakkos, J. Boronat, J. Casulleras, and S. Giorgini, Equation of state of an interacting Bose gas at finite temperature: A path-integral Monte Carlo study. Physical Review A 74, 043621 (2006).

[34] J. K. Nilsen, J. Mur-Petit, M. Guilleumas, M. Hjorth-Jensen, and A. Polls, Vortices in atomic Bose-Einstein condensates in the large-gasparameter region. Physical Review A 71, 053610 (2005). 
[35] J. L. DuBois, and H. R. Glyde, Bose-Einstein condensation in trapped bosons: A variational Monte Carlo analysis. Physical Review A 63, 023602 (2001).

[36] S. Y. Chang, Radio-frequency response of strongly interacting Fermi gases at finite temperatures. Physical Review A 80, 033623 (2009).

[37] S. Y. Chang, Equation of state and phases of a polarized unitary Fermi gas. Physical Review A 77, 051602(R) (2008).

[38] G. Pupillo, A. Griessner, A. Micheli, M. Ortner, D.-W. Wang, and P. Zoller, Cold Atoms and Molecules in Self-Assembled Dipolar Lattices. Physical Review Letters 100, 050402 (2008).

[39] H. P. Bü̈chler, E. Demler, M. Lukin, A. Micheli, N. Prokof'ev, G. Pupillo, and P. Zoller, Strongly Correlated 2D Quantum Phases with Cold Polar Molecules: Controlling the Shape of the Interaction Potential. Physical Review Letters 98, 060404 (2007).

[40] T. Lahaye, C. Menotti, L. Santos, M. Lewenstein, and T. Pfau, The physics of dipolar bosonic quantum gases. Reports on Progress in Physics 72, 126401 (2009).

[41] Y. Y. Lin, R.-K. Lee, Y.-M. Kao, and T.-F. Jiang, Band structures of a dipolar Bose-Einstein condensate in one-dimensional lattices. Physical Review A 78, 023629 (2008)

[42] J. L. Bohn, R. M. Wilson, and S. Ronen, How does a Dipolar BoseEinstein Condensate Collapse?. Laser Physics 19, 547 (2009).

[43] K. Góral, K. Rzążewski, and T. Pfau, Bose-Einstein condensation with magnetic dipole-dipole forces. Physical Review A, 61, 051601(R) (2000).

[44] K. Góral and L. Santos, Ground state and elementary excitations of single and binary Bose-Einstein condensates of trapped dipolar gases. Physical Review A, 66, 023613 (2002).

[45] T. Koch, T. Lahaye, J. Metz, B. Froehlich, A. Griesmaier, and T. Pfau, Stabilization of a purely dipolar quantum gas against collapse. Nature Physics 4, 218, (2008).

[46] S. Sachdev, Quantum Phase Transitions. Cambridge University Press, 1999. 
[47] J. M. Kosterlitz, and D. J Thouless, Ordering, metastability and phase transition in two-dimensional systems. Journal of Physics C 6, 1181 (1973).

[48] T. D. Küner, S. R. White, and H. Monien, One-dimensional BoseHubbard model with nearest-neighbor interaction. Physical Review B, 61, 12474 (2000).

[49] S. Wessel, F. Alet, M. Troyer and G.G. Batrouni, Quantum Monte Carlo simulations of confined bosonic atoms in optical lattices. Physical Review A, 70053615 (2004).

[50] J.K. Freericks, and H. Monien, Strong-coupling expansions for the pure and disordered Bose-Hubbard model. Physical Review B, 532691 (1996).

[51] W. Krauth, M. Caffarel, and J.-P. Bouchaud, Gutzwiller wave function for a model of strongly interacting bosons. Physical Review B, 45, 3137 (1992).

[52] D. S. Rokhsar, and B. G. Kotliar, Gutzwiller projection for bosons. Physical Review B, 44, 10328 (1991).

[53] W. Zwerger, Mott-Hubbard transition of cold atoms in optical lattices. Journal of Optics B, 5, S9, (2003).

[54] D. Jaksch, V. Venturi, J. I. Cirac, C. J. Williams and P. Zoller, Creation of a Molecular Condensate by Dynamically Melting a Mott Insulator. Physical Review Letters, 89, 040402, (2002).

[55] D. Jaksch, C. Bruder, J. I. Cirac, C. W. Gardiner, and P. Zoller, Cold Bosonic Atoms in Optical Lattices. Physical Review Letters 81, 3108 (1998).

[56] C. C.-Tannoudji, B. Diu, F. Laloë, Quantum Mechanics. John Wiley \& Sons, 1977.

[57] C. Menotti, C. Trefzger, and M. Lewenstein, Metastable States of a Gas of Dipolar Bosons in a 2D Optical Lattice. Physical Review Letters, 98, 235301 (2007).

[58] C. Trefzger, C. Menotti, and M. Lewenstein, Ultracold dipolar gas in an optical lattice: The fate of metastable states. Physical Review A, 78, 043604, (2008). 
[59] X.-G. Wen, Quantum field theory of many-body systems. Oxford University Press, Oxford, 2004.

[60] V. M. Pérez-García, H. Michinel, J. I. Cirac, M. Lewenstein, and P. Zoller, Low Energy Excitations of a Bose-Einstein Condensate: A TimeDependent Variational Analysis. Physical Review Letters 77, 5320 (1996).

[61] V. W. Scarola, E. Demler, and S. Das Sarma, Searching for a supersolid in cold-atom optical lattices. Physical Review A 73, 051601(R) (2006).

[62] E. Altman, E. Demler, and M. D. Lukin, Probing many-body states of ultracold atoms via noise correlations. Physical Review A 70, 013603 (2004).

[63] S. Fölling, F. Gerbier, A. Widera, O. Mandel, T. Gericke, and I. Bloch, Spatial quantum noise interferometry in expanding ultracold atom clouds. Nature 434, 481 (2005).

[64] W. S. Bakr, J. I. Gillen, A. Peng, S. Fölling, M. Greiner, A quantum gas microscope - detecting single atoms in a Hubbard regime optical lattice. arXiv:0908.0174.

[65] A. Argüelles, and L. Santos, Mott-insulator phases of nonlocally coupled one-dimensional dipolar Bose gases. Physical Review A 75, 053613 (2007).

[66] G.-H. Chen and Y.-S. Wu, Quantum phase transition in a multicomponent Bose-Einstein condensate in optical lattices. Physical Review A 67, 013606 (2003).

[67] D.-W. Wang, M. D. Lukin, and E. Demler, Quantum Fluids of SelfAssembled Chains of Polar Molecules. Physical Review Letters 97, 180413 (2006).

[68] M. Klawunn, J. Duhme and L. Santos, Bose-Fermi mixtures of selfassembled Plaments of fermionic polar molecules. arXiv:0907.4612.

[69] L. Mathey, I. Danshita, and C. W. Clark, Creating a supersolid in onedimensional Bose mixtures. Physical Review A 79,011602(R) (2009).

[70] D.-W. Wang, Quantum Phase Transitions of Polar Molecules in Bilayer Systems. Physical Review Letters 98, 060403 (2007).

[71] S. Yi, T. Li, and C.P. Sun, Novel Quantum Phases of Dipolar Bose Gases in Optical Lattices. Physical Review Letters 98, 260405 (2007). 
[72] D.-W. Wang, and E. Demler, Collective excitations and instabilities in multi-layer stacks of dipolar condensates. arXiv:0812.1838

[73] M. Klawunn, and L. Santos, Hybrid multi-site excitations in dipolar condensates in optical lattices. arXiv:0812.3543

[74] C. Trefzger, C. Menotti, and M. Lewenstein, Pair-Supersolid Phase in a Bilayer System of Dipolar Lattice Bosons. Physical Review Letters, 103, 035304, (2009).

[75] B. L. Lev, E. R. Meyer, E. R. Hudson, B. C. Sawyer, J. L. Bohn, and J. Ye, OH hyperfine ground state: From precision measurement to molecular qubits. Physical Review A, 74, 061402(R) (2006).

[76] B. C. Sawyer, B. L. Lev, E. R. Hudson, B. K. Stuhl, M. Lara, J. L. Bohn, and J Ye, Magnetoelectrostatic Trapping of Ground State OH Molecules. Physical Review Letters, 98, 253002 (2007).

[77] E. Altman, W. Hofstetter, E. Demler and M. D. Lukin, Phase diagram of two-component bosons on an optical lattice. New Journal of Physics, 5, 113 (2003).

[78] A. B. Kuklov and B.V. Svistunov, Counterflow Superfluidity of TwoSpecies Ultracold Atoms in a Commensurate Optical Lattice. Physical Review Letters, 90, 100401 (2003).

[79] C. C.-Tannoudji, J. D.-Roc, and G. Grynberg, Atom-Photon Interactions. Wiley, New York, 1998.

[80] M. Boninsegni, and N.V. Prokof'ev, Phase diagram of an anisotropic bosonic t-J model. Physical Review B 77, 092502 (2008).

[81] N. V. Prokof'ev, B. V. Svistunov, and I. S. Tupitsyn, "Worm" algorithm in quantum Monte Carlo simulations. Physics Letters A 238, 253 (1998).

[82] N. V. Prokof'ev, B. V. Svistunov, and I. S. Tupitsyn, Exact, complete, and universal continuous-time worldline Monte Carlo approach to the statistics of discrete quantum systems. Journal of Experimental and Theoretical Physics 87, 2 (1998).

[83] B. Capogrosso-Sansone, N. V. Prokof'ev, and B. V. Svistunov, Phase diagram and thermodynamics of the three-dimensional Bose-Hubbard model. Physical Review B 75, 134302 (2007). 
[84] E. L. Pollock, and D. M. Ceperley, Path-integral computation of superfluid densities. Physical Review B, 36, 8343 (1987).

[85] T. Gericke, P. Würtz, D. Reitz, T. Langen, and H. Ott, High-resolution scanning electron microscopy of an ultracold quantum gas. Nature Physics 4, 949-953 (2008).

[86] B. Capogrosso-Sansone, C. Trefzger, M. Lewenstein, P. Zoller, and G. Pupillo, Quantum Phases of Cold Polar Molecules in 2D Optical Lattices. arXiv:0906.2009. Accepted for Physical Review Letters publication. 